LA-8030-T

Thesis

\title{
A LAMPF Neutrino Experiment to Test Muon Number Conservation
}

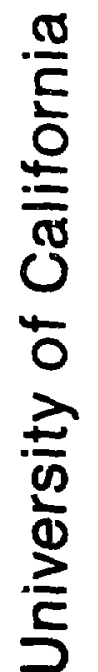


LAP030.T

Thenis

Uc-34

Isered: Septumber 197:

\section{A LAMPF Neutrino Experiment to Test Muon Number Conservation}

Suzanne Eileon Willis*

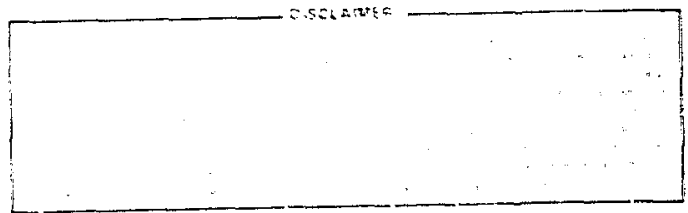

-Physics Department, Fermi Natiorial Accelerator Laboratory. Box 500, Batavia, IL 60510.

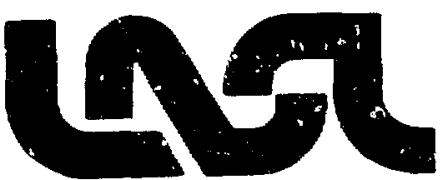


TABLE OF CONTENTS

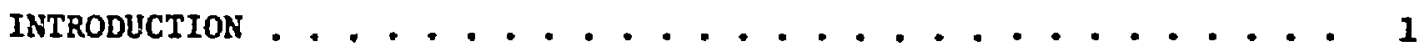

CHAPTER 1. THEORY. ................... 6

A. NORMAL LEPTON INTERACTIONS ............ 6

1. Beta Decay. ............... 6

2. Muon Decay. ............... 10

3. Inverse Beta Decay ............. 16

B. GAUGE THEORIES ..................... 19

1. An Example: Quantum Electrodynamics as a Theory ............ 19

2. The Welnberg-Salam Model ......... 25

C. THEORETICAL CONSIDERATIONS OF $\bar{v}_{\mathrm{e}}$ FROM $\mu^{+}$DEC $\ldots \ldots \ldots$

1. Multiplicative Law ............ 32

2. Neutrino Oscillations and Decay ......... 37

CHAPTER 2. EMPERIMENTAL apParatus ............. 40

A. CERENKOV COUNTER .................. 40

B. SCINTILLATORS ................. 60

c. DRIFT CHAMBERS ................ 68

CHAPTER 3. THE LAIPF ACCELERATOR AND TIE NEUTRINO HOUSE . . . . 70

A. THE LAMPE ACCELERATOR. ............. 70

B. THE NEUTRINO HOUSE . . . . . . . . . . . . 73 CHAPTER 4. BACKGROUNDS ........................... 76

A. NON-BEANIA SOCLATEN BACKGROUNDS ......... 76

B. BEAHTASST,LTATED BACKGROUNDS ........... 85

CHAPTER 5. ELECTRONICS . . . . . . . . . . . . 99 
CHAPTER 6. DATA ACQUISITION . . . . . . . . . . 106 A. DATA ACQUISITION ......................... 106

B. Calibrations and checks ............. 215

C. HISTORY OF THE EXPERIMENT . . . . . . . . . . 119 CHAPTER 7. anALYSIS .................. 123 CHAPTER 8. RESULTS AND CONCLUSIONS ............. 131 A. HISTORY OF DATA TAKING ..................... 131 B. HEAVY WATER RESULTS ............... 133 c. hater RESULTS ................. 136 D. SYSTEMATTC EFFECTS . . . . . . . . . 137

E. CONCLUSIONS ................ 143 ACKNOWLEDGMENTS . . . . . . . . . . . . . . . . . . . 148 


\section{LIST. OF TABLES}

Table I.

Table II.

Table III.

Table IV.

Table Y.

Table VI.

Table VII.

Table VIII.

Table IX.

Table X.

Table XI.

Table XII.

Table XIII.

Table XIV.

Table XV.

Table XVI.
Lepton Number Assignment . . . . . . . . .

Muon Number Assignment ...... . . . . 33

Diffuse Reflectivity of Various Samples . . . . 51

Gain of the Cerenkov Counter as a Function of

Reflector and Wavelength Shifter . . . . . . 59

Nomenclature of Scintillators ......... 65

Rat lo of Neutron Backgrounds, Blomed Target In/

Target Out, as a Function of Energy . . . . . . 94

CAMAC Coincldence Reglster Word ........ 105

CAMAC Scalers ............... . 111

Scaler Warnings . . . . . . . . . . . 112

Spectrum Definitions . . . . . . . . . 116

Tape Format . . . . . . . . . . . . 124

$\mathrm{D}_{2} \mathrm{O}$ Results Cycle by Cycle ........... 133

$\mathrm{D}_{2} \mathrm{O}$ Results as a Function of $\mathrm{BIn}$ S1ze and

Analysis Region ... . . . . . . . . . 134

$\mathrm{H}_{2} \mathrm{O}$ Results Cycle by Cycle . . . . . . . . 136

$\mathrm{H}_{2} \mathrm{O}$ Results as a Function of $\mathrm{Bin}$ Size and Analysis

Region . . . . . . . . . . . . . . 137

Calculation of $\mu^{-} / \mu^{+}$Ratio in Copper Beam Stop . . 144 


\section{LIST OF FIGURES}

Figure 1. Cerenkov Counter ............... 42

Figure 2. Counter Assembly and Aluminum Frame . . . . . . . 43

Eigure 3. Orllentaliton of Struts . . . . . . . . . . . 45

Figure 4. Porthole Ring ................. . 46

Figure 5. Followir Ring . . . . . . . . . . . . . 47

Figure 6. Phototube Assembly . . . . . . . . . . . . . 48

Figure 7. Refle:tor Test Setup . . . . . . . . . . . 50

Figure 8. Water Manifolds . . . . . . . . . . . 52

Figure 9. Umbelliferone Concentration Curve . . . . . . . 55

Figure 10. Circuit Diagram, EMI 9618R Phototube Base . . . . 58

Figure 11. Scintillator, Light Pipe, and $90^{\circ}$ Bend Frisns . . . 61

Figure 12. Side Scintillators, Horizontal . . . . . . . . . 62

Figure 13. Side ScIntillators, Vertical . . . . . . . . . 63

Figure 14. Top View, Scintillator Stands . . . . . . . . . . 64

Figure 15. Top Scintillators . . . . . . . . . . . 66

Figure 16. Circult Dlagram, RCA 4525 Phototube Base . . . . . 67

Figure 17. Exploded View of Experimental Setup . . . . . . . 69

Figure 18. LAMPF Experimental Area . . . . . . . . . . . 71

Figure 19. Neutrino House Area . . . . . . . . . . . . 74

Figure 20. Muon Bremsstraklung . . . . . . . . . . . 79

Figure 21. Mun Decay with Electron Bremsstrahlung . . . . . 81

Figure 22. Position of South Drift Chamber . . . . . . . . 82

Figure 23. Spectrum Shape of Cosmic-Ray Backgrounds . . . . . . 84 
Figure 24. Thermal Neutrons, Biomed Target Out, $i_{2} 0$. . . . 87

Figure 25. Thermal Neutrons, Biomed Target In, $\mathrm{H}_{2} \mathrm{O} \ldots . . .88$

Figure 26. Thermal Neutrons, Biomed Target Out, $\mathrm{H}_{2} \mathrm{O}$ with

Calculated Neutrino Spectrum .......... 89

Figure 27. Thermal Neutrons, Biomed Target $\mathrm{In}, \mathrm{H}_{2} \mathrm{O}$, with

Calculated Meutrino Spectrum .......... 90

Figure 28. Beam-Associated Neutrals Rate vs Shielding Thickness . 92

Figure 29. Spectrum Shape, Beam-Associated Neutra1s, 5-m

Shielding, Biomed Target Out .......... 93

Figure 30. Relative Cross Sections of $\bar{v}_{e} p, \nu_{e} d, v_{e}{ }^{12} C$, and $v_{e}{ }^{16} 0$ as a Function of Energy .......... 95

Figure 31. Thermal Neutrons, Biomed Target Out, $\mathrm{D}_{2} \mathrm{O} \ldots . . .97$

Figure 32. Thermal Neutrons, Biomed Target In, $D_{2} \mathrm{O} \ldots \ldots 9$

Figure 33. Electronics Block Diagram ........... 130

Figure 34. Flow Chart, Data-Acquisition Program . . . . . 107

Figure 35. Beam and Gate Timing ............ 108

Figure 36. Run Sheet .............. 113-114

Figure 37. Measured and Calculated Muon Decay Spectra . . . 118

Figure 38. Contour Plots, DCRs vs Energy for Muon Decays

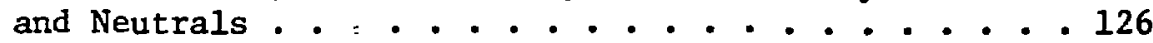

Figure 39. Calculated spectra, $e^{+}$from $\bar{\nu}_{e} P \rightarrow n e^{+}, e^{-}$from

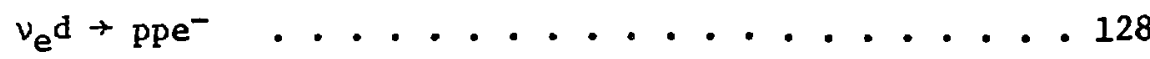

Figure 40. $\mathrm{D}_{2} \mathrm{O}$ Subtracted Spectrum with Calculated Spectrum . 135

Figure 41. $\mathrm{H}_{2} \mathrm{O}$ Subtracted Spectrum with Calculated Spectrum . 138

Figure 42. $\mathrm{H}_{2} \mathrm{O}$ Spectrum with Neutrino-Nucleus and Neutron

Backgrounds Subtracted, with Calculated Spectrum ... 139

Figure 43. $D_{2} O$ Spectrum with Neutrino-Nucleus and Neutron

Backgrounds Subtracted, with Calculated Spectrum . . 141

Figure 44. Probability of observing $\bar{\nu}_{e}$ as a Function of $M_{1} \ldots 146$ 


\author{
ABSTRACT \\ A LAMPF NEUTRINO EXPERIMENT TO TEST \\ MUON TIMBER CONSERVATION \\ Suzanne Eileen Willis \\ Yale University 1979
}

The need for a muon number conservation law separate from lepton number conservation is mandated by the absence of neutrinoless muon electron transitions. The form of the muon number conservation law, however, is not determined by these considerations. A search has been made for electron antineutrinos coming from positive muon decays, which would be allowed by a multiplicative formulation of muon number conservation, but not by an additive formulation.

The LAMPF beam dump was used as a source of positive mions decaying at rest; electron antineutrinos were detected via inverse beta decay : n a six-ton water Cerenkov counter. No excess of events above known backgrounds was seen; a lim:t was placed on the branching ratio for positive muon decay to electron antineutrinos of $\mathrm{R}<6.5 \%$.

Electron neutrinos from normal muon decay were detected during a fill of the counter with heavy water, via the inverse beta decay on the deuteron. The cross section for this reaction was measured, averaged over the neutrino energy spectrum; the measured value of $(0.52 \pm 0.18)$ $\times 10^{-40} \mathrm{~cm}^{2}$ is in good agreement with the calculated value of $0.48 \times 10^{-40} \mathrm{~cm}^{2}$. 


\section{INTRODUCTION}

The need for a muon number conservation law separate from lepton number conservation has been recognized for many years. The need for this law is mandated by the absence of Juon-electron transitions without neutrinos, such as

$$
\begin{gathered}
\mu^{+}+e^{+} \gamma \\
\mu^{+}+e^{+} e^{+} e^{-} \\
\mu^{-} z+e^{-} z
\end{gathered}
$$

and also by the observation of the distinct identity of the neutrino from pion decay

$$
\pi^{+}+\mu^{+} v_{\mu}
$$

and that from beta decay

$$
n+p e^{-} \bar{v}_{e}
$$

such that

$$
v_{\mu} \mathrm{z}, \mathrm{e}^{-} \mathrm{z}^{\prime}(\operatorname{Ref} \cdot 1),
$$

1. Danby et al., Phys. Rev. Lett. 2, 36 (1962). 
The exact form of the muon number conservation law is not determined by the above considerations; it was pointed out by Feinberg and Weinberg ${ }^{2}$ that muon number could be conserved either additively or multiplicatively. Defining

$$
\begin{aligned}
\mathrm{L}_{\mu} & =+1 \text { for } \mu^{-}, \nu_{\mu} \\
& =-1 \text { for } \mu^{+}, \bar{\nu}_{\mu} \\
& =0 \text { for all others, }
\end{aligned}
$$

the additive formulation requires that $\Sigma\left(L_{\mu}\right)=$ constant, while the multiplicative formulation requires $\Pi(-1)^{L_{\mu}}=$ constant. Both formulations forbid direct $\mu-e$ transitions as detailed above, but they are not identical. The additive law is more restrictive concerning reactions which involve at least two electrons and two muons. Examples of reactions which are allowed by the multiplicative formulation but not by the additive one include

$$
\begin{aligned}
& \mu^{+} e^{-}+\mu^{-} e^{+} \\
& e^{-} e^{-}+\mu^{-} \mu^{-} \\
& \mu^{+}+e^{+} \nu_{e} \nu_{\mu} .
\end{aligned}
$$

The third reaction is muon decay with inverted neutrinos; the normal decay, allowed by both formulations, is

$$
\mu^{+}+e^{+} \nu_{e} \bar{\nu}_{\mu}
$$

2. G. Feinberg and S. Weinberg, Phys. Rev. Lett. 6 , 381 (1961). 
Present 1inlts on the first two reactions are considerably larger than the rate expected from weak interactions. 3 Previous limis on the muon decay to inverted neutrinos come from the Gargamelle collaboration at CERN; defining the branching ratio

$$
R \equiv \frac{\mu^{+}+e^{+} \bar{v}_{e} v_{\mu}}{\mu^{+}+a 11},
$$

the Gargame11e collaboration found $R<0.25$ (90\% C.L.) in 1973,4 using 52 events. More detalled analys1s, also by the Gargamelle collaboration, of a larger sample of 260 events yielded $R=0.13 \pm 0.15$ in 1978.5

The above formulation of multiplicative muon number conservation does not constitute a complete theory; in particular, there is no Indication of what to expect for a branching ratio. However, a finfte result for $R$ would be evidence of a multiplicative law.

We have performed an experiment at the Los Alamos Meson Physics Facility (LAMPF) to search for both $\nu_{e}$ and $\bar{\nu}_{e}$ from $\mu^{+}$decay via the inverse beta decay reactions

$$
\begin{aligned}
& v_{e^{d}}+\mathrm{ppe}^{-} \\
& \vec{v}_{\mathrm{e}} \mathrm{p} \rightarrow \mathrm{ne}^{+}
\end{aligned}
$$

3. R. Amato et al., Phys. Rev. Lett. 21, 1709 (1968);

W.C. Barber et a1., Phys. Rev. Lett. 22, 902 (1969).

4. Eichten et al., Phys. Lett. 46B, 281 (1973).

5. Blfetschau et a1., Nucl. Phys. B133, 205 (1978). 
In $81 x$-ton water Cerenkov counter which was filled with efther $\mathrm{H}_{2} \mathrm{O}$ or $\mathbf{D}_{2} \mathrm{O}$

Our scurce of $\mu^{+}$was the beam dump for the priary proton beam. IAPF is an $800-\mathrm{MeV}$ Iinear accelerator which accelerates both protons and $H^{*}$ ions. Design intengity for the proton beam 1 s 1 wh at a 67 duty factor. The beam dump was vater-cooled copper; protons stopping in it produced both $\pi^{+}$and $\pi^{-}$. The $\pi^{-}$were captured on nuclel; the $\pi^{+}$were stopped in the copper and decayed:

$$
\pi^{+}+\mu^{+} v_{\mu}
$$

The $\mu^{+}$also stopped and decayed; this was our source of muons. The $\mu^{-}$ contamination (from $\pi^{-}$decay) has been estimated to be $1 / 7000$; $^{6}$ it will therefore not contribute at our expected level of sensitivity, a few percent. Our detector was situated at $90^{\circ}$ to the beam direction, behind $6.3 \mathrm{~m}$ of steel shielding. The neutrinos emerged isotropicaliy from the se im dump, but high-energy neutrons tended to go forward; we chose $90^{\circ}$ to minimize neutron backgrounds. We measured beam-assocaited backgrounds wtth smaller amounts of shielding between our detector and the beam dump; the final amount of $6.3 \mathrm{~m}$ was chosen as being sufficient to artenuate beam-associated background events so they were small compared to our expected level of sensitivity. The detector was surrounded on all sides by at least $1 \mathrm{~m}$ of shielding to reduce backscattered neutrons and cosmic-ray events.

6. LAMPF Neutrino Facility Proposal, Los Alamos Scientific Laboratory report LA-4842-MS (1971). 


\section{$-5-$}

The detector was surrounded by a 4n cosic-ray anticollacidence shleid, consisting of an Inner layer of sclntillatora, a layer of lead, and an outer layer of active counters (scintillators on top, drift chasbers on the sides). This anticoincidence shield reduced the coseic-ray backgrounds in the counter in $(20,60)$ veV by a factor of $10^{3}$. The reasining cosalc-ray events could be measured accurately on Iine and subtracted directiy, because of the tine structure of the bean. The LAMPF duty factor was 67 , with a 500-us bear ap111 and a repetition rate of $120 \mathrm{hz}$. The bean spill was long compared to the mon iffettre, so we required events to occur duriag the sp111. This had the 1raediate effect of reducing the cosalc-ray background to 67 of the total rate, and enabled us to open a gate betveen spllis which vas long comared to the length of the spill, so we could accurately weasure the coenic-ray contribution and subtract 1t. The cosnic-ray events then did not contribute to the subtracted spectrue except in the uncertalnty of the subtraction. The level of sensitivity achleved by the experinent then depended on the amount of running tiat, to reduce the error on the subtraction, and on the intensity of the beam, to Increase the signal to nolse ratio. 


\section{CHAPTER I}

\section{THEORY}

\section{A. NORMAL LEPTON INTERACTIONS}

\section{Beta Decay}

The Fermi theory of beta decay is the basis for severa' asperts of this experiment. Neutrinos are detected via the inverse beta decay process on protons, and muon decay is closely related to beta decay. These processes, inverse beta decay and muon decay, w111 be discussed specifically below. The discussion will begin with the simple theory of beta decay, to lay the groundwork for what follows. This discussion follows Wu and Moskowski. 7

We assume that the beta decay from an arbitrary nucleus can be represented by ${ }^{8}$

$$
z^{A}+(z+1)^{A}+e^{-}+\bar{v}_{e}
$$

or, In the case of positron decay,

$$
z^{A}+(z-1)^{A}+e^{+}+v_{e}
$$

We will assume that the interaction is a point interaction, that is, that all four particles interact at a point, with no intermediaries.

$\overline{7 . ~ W u ~ a n d ~ M o s k o w s k i, ~ B e t a ~ D e c a y, ~ J o h n ~ W l l e y ~ a n d ~ S o n s, ~} 1966$.

8. IbId., Pp. 21-24. 
This corresponds to an infintte intermediace vector boson wase. This leads to a Kaniltonian of the form

$$
H_{B}=g \sum_{n}\left[\psi_{e}^{*}\left(t_{n}\right) \psi_{v_{v}}^{*}\left(t_{n}\right) Q_{n}^{+}+\psi_{e}\left(t_{n}^{+}\right) \psi_{v_{e}}\left(\vec{x}_{n}\right) Q_{n}^{-}\right],
$$

where the surs is over all particles in the nucleus. The constant $g$ gives the strength of the interaction. The wave functions $v_{e}^{\star}\left(\vec{r}_{n}\right)$ and $\psi \vec{v}_{e}\left(\vec{r}_{n}\right)$ are the created eiectron and neutrino at the position of the $n^{\text {th }}$ nucleon, coupled to the operator $Q_{n}^{+}$whtch changes a neutron Into a proton but otherwise leaves its wave function unchanged. Then we have the wave functicns $\dot{\psi}_{\mathrm{e}}\left(\vec{x}_{n}\right), \psi_{\bar{v}_{\mathrm{e}}}\left(\vec{r}_{n}\right)$ for the annihtlated leptons, counled to $\mathrm{O}_{n}^{-}$which changes a proton to a neutron. The neutrino wave function 1. just a plane wave; the electron wave function is not, to the extent that it interacts with the Coulonb field of the nucleus and surrounding e lectrons.

The effects of spin will now be added to this formulation. 9 Results wil be discussed in terms of the Hamiltonian density, $\stackrel{H}{=}=\psi_{\mathrm{P}}^{\dagger_{B}} \psi_{\mathrm{n}}$, rather than the Hawiltonian itself. The wave functions of each particle will now be two-component spinors. There are four bilinear combinations of the electron and neutrino ware functions:

$$
\psi_{e}^{\psi}, \psi_{e} \dagger_{\sigma_{v}}, \psi_{\mathrm{e}} \dagger_{\mathrm{y}} \psi_{v}, \dot{\psi}_{\mathrm{e}} \dagger_{z} \psi_{v}
$$

where $o_{x, y, z}$ are the Pauli spin matrices:

$$
\sigma_{x}=\left(\begin{array}{ll}
0 & 1 \\
1 & 0
\end{array}\right) ; \sigma_{y}=\left(\begin{array}{rr}
0 & -i \\
i & 0
\end{array}\right): \sigma_{z}=\left(\begin{array}{rr}
1 & 0 \\
0 & -1
\end{array}\right) \text {. }
$$

9. Ibid., PP $\cdot 43-51$. 
The flrat combination is a scalar, and the other three form an axial vector. The only way to combine these terms to yleld a scalar is in the combinations $S \cdot S$ or $A \cdot A$; keeping only tering which are invariant under rotation giveg two allowed forms of the interaction:

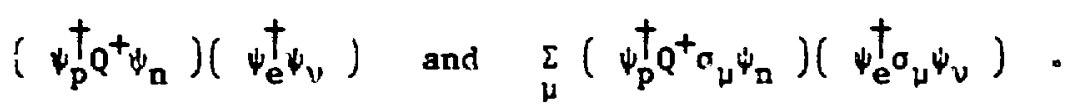

Iri the relativistic formulation, the particles obey the Dirac equation:

$$
\left(\gamma_{\mu} p^{\mu}-m\right) \psi=0
$$

where $h=c=1$, and $\gamma_{\mu}$ are the Dirac matrices. A standard representation is

$$
\gamma_{0}=\gamma^{0}=\left(\begin{array}{cc}
I & 0 \\
0 & -I
\end{array}\right) ; \gamma^{1}=\left(\begin{array}{cc}
0 & \sigma_{i} \\
-\sigma_{1} & 0
\end{array}\right),
$$

and their wave functions are now four-component spinors. Once again Invokjng rotational invarłance, and adding lnvariance under proper Lorentz transformations and space 1nversion (parity violation will be discussed shortly) ylelds five types of interaction, the scalar, tensor, pseudoscalar, vector, and axial vector forms, each with its own coupling constant. These forms are named for the way in which the nucleon part of the equation transforms. Assuining a nonrelativistic treatment of the nucleons yields: 


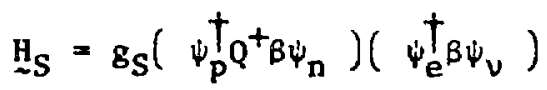

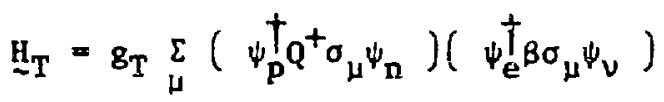

$$
\begin{aligned}
& \underline{\underline{I}}_{\mathrm{P}}=0
\end{aligned}
$$

(This cerm is zero because it contains a term of the form $\psi_{p}^{\dagger} \gamma_{5} \psi_{n}$, which vanishes in the Iimit of low nucleon velociries.)

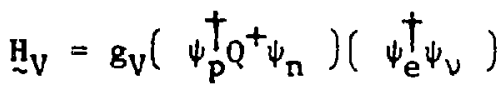

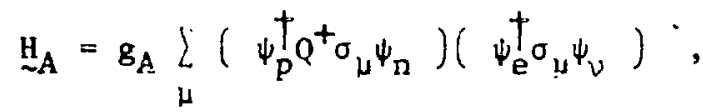

where $B=\left(\begin{array}{rr}I & 0 \\ 0 & -I\end{array}\right)$.

In order to take the parity nonconserving terms into account, it 19 necessary to introduce a pseudoscalar interaction into the Hamiltonian density, as well as the original scalar interaction: 10

$$
\begin{aligned}
\underset{H}{H}= & \underset{1}{[}\left[\mathrm{C}_{i}\left(\tilde{\psi}_{\mathrm{p}} \hat{o}_{i} Q^{+} \psi_{n}\right)\left(\tilde{\psi}_{e} \hat{o}_{i} \psi_{\nu}\right)\right. \\
& +\sum_{1} c_{i}^{\prime}\left(\psi_{p} \hat{o}_{i} Q^{+} \psi_{n}\right)\left(\psi_{e} \hat{o}_{i} \gamma_{5} \psi_{\nu}\right) \\
& +h \cdot c \cdot],
\end{aligned}
$$

where $i=S, T, P, V, A, \quad g_{1}=g_{2}, \hat{o}_{1}$ are the particular operators given above, and h.c. means hermitian conjugate. The famous experiment of Wu 
et al.11 found $C_{1}=c_{i}$ (for the axial vector part of the interaction). Th1s leads directly to the two-component theory of the neutrino, 12 assuming the neutrino 1 massless; since the neutrinos are completely polarized and exhibit only one helicity state, only two components of the spinor are needed to describe them, rather than four.

\section{Mison Decay}

We will now consider muon decay, $\mu^{+} \rightarrow e^{+} v_{e} \bar{\nu}_{\mu^{\circ}} 13$ The Hamiltonian density for this decay is analogous to the one for beta decay:

$$
g_{1}\left(\tilde{\psi}_{e} \hat{o}_{1} \psi_{\mu}\right)\left(\tilde{\psi}_{\nu} \hat{o}_{1} \psi_{\nu}\right)
$$

If we now use the two-component wave functions for the neutrinos, we have for the neucrino part:

$$
\frac{1}{4} g_{i}\left[\tilde{\psi}_{\nu_{\mu}}\left(1 \mp \gamma_{5}\right) \hat{0}_{i}\left(i \pm \gamma_{5}\right) \psi_{\nu_{e}}\right]
$$

For the S, T, and $P$ interactions, $\hat{o}_{i}$ is a sum of terms containing an even number of gamma matrices. Therefore, $\hat{o}_{1}$ and $\gamma_{5}$ commute and the terms vanish. For the $V$ and $A$ interactions, $\hat{o}_{1}$ is a sum of terms containing an odd number of gamma matrices, and will anticommute with $\gamma_{5}$; this term therefore becomes

$$
\frac{1}{2} g_{1}\left[\tilde{\psi}_{\nu_{\mu}} \hat{o}_{1}\left(1+\gamma_{5}\right) \psi_{\nu_{e}}\right]
$$

11. C. S. Wu, E. Ambler, R. W. Haywarä, D. D. Hoppes, and R. F. Hudson, Phys. Rev. 105, 1413 (1957).

12. Wu and Moskowsk1, op. c1t

13. Ibid., Pp. 212-213. 
The muon decay must therefore be a combination only of $V$ and $A$ Interactions. Experiments Indicate ${ }^{14}$ that the form $18 \mathrm{~V}-\mathrm{A}$.

We will now consider the derivation of the $V-A$ form from chirality Invariance. 15 Chirality is defined as the transformation $\psi$. $\gamma_{5} \psi$. For the neutrino, which is massless, the wave functions are eigenfunctions of chirality (we speak here of the four solutions to the Lirac equation For a particle of a given momentum), two positive, $\gamma_{5} \psi=\psi$, and two negative, $\gamma_{5} \psi=-\psi$. For massive particles, the wave functions are not eigenstates of chirality, but can be expanded in terms of these eigenstates. We then make the assumption that the four-fermion interaction should be invariant under chirality transformations on any of the wave functions. In order for this to be true, using the form of the interaction

$$
\frac{1}{\sqrt{2}} g_{1}\left(\tilde{\psi}_{2} \hat{o}_{i} \psi_{1}\right)\left(\tilde{\psi}_{4} \hat{o}_{i} \psi_{3}\right) \text {, }
$$

it is necessary for $\hat{0}_{1}$ and $\gamma_{5}$ to anticommute. As shown above, the only interactions for which this is true are $V$ and $A$ :

$$
\hat{0}=a \gamma_{\mu}+b \gamma_{\mu} \gamma_{5}
$$

Now, from the condition

$$
\hat{o} \gamma_{5}=\hat{0}, \hat{o}=a \gamma_{\mu} \gamma_{5}+b \gamma_{\mu}=a \gamma_{\mu}+b \gamma_{\mu} \gamma_{5},
$$

14. Ibid., Pp $250-252$.

15. Ibid., p. 224 . 
It follows that $a=b$;

$$
\hat{0}=a \gamma_{\mu}\left(1+\gamma_{5}\right)
$$

The Interaction then becomes

$$
\frac{1}{\sqrt{2}} \mathrm{~g}\left[\tilde{\psi}_{2} \gamma_{\mu}\left(1+\gamma_{5}\right) \psi_{1}\right]\left[\tilde{\psi}_{4} \gamma_{\mu}\left(1+\gamma_{5}\right) \psi_{3}\right]
$$

Writing this explicitly for $\mu^{-}$decay, and indicating the sum over gamma Indices, we have

$$
\underset{\mathrm{H}}{=}=\frac{1}{\sqrt{2}} \underset{\mu}{\mathrm{g}} \tilde{\psi}_{\nu_{\mu}} \gamma_{\mu}\left(1+\gamma_{5}\right) \psi_{\mu} \tilde{\psi}_{\mathrm{e}} \gamma_{\mu}\left(1+\gamma_{5}\right) \psi_{\nu_{e}} \text {. }
$$

We can now calculate the electron energy spectrum, 16 which will be the same as the positron spectrum from $\mu^{+}$decay. This will also be the same as the antineutrino spectrum from $\mu^{+}$decay because the helicity of the antineutrino (whether it is $\bar{v}_{e}$ or $\bar{v}_{\mu}$ ) is the same as the helicity of the positron. We have

$$
\left(1+\gamma_{5}\right) \psi_{\nu}=2 \psi_{\nu}, \tilde{\psi}_{\nu_{\mu}} \gamma_{\mu}\left(1+\gamma_{5}\right)=2 \tilde{\psi}_{\nu_{\mu}} \gamma_{\mu}
$$

Then

$$
\underline{H}=\sqrt{8 g} \sum_{\mu} \tilde{\psi}_{\nu} \gamma_{\mu} \psi_{\mu} \tilde{\psi}_{\mathrm{e}} \gamma_{\mu} \psi_{\nu}
$$


Using the two-particle wave functions written in two-component form,

$$
\begin{aligned}
& \psi_{\mu}=\left(\begin{array}{l}
x_{\mu} \\
0
\end{array}\right)
\end{aligned}
$$

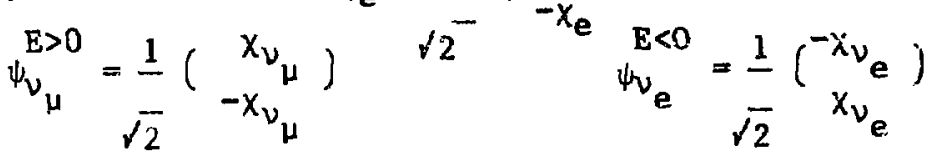

where

$$
x_{\nu_{\mu}}=\left(\begin{array}{l}
\cos ^{1} / 2 \theta_{\nu_{\mu}} \\
\sin ^{1} / 2 \theta_{\nu_{\mu}} e^{i \phi_{\nu_{\mu}}}
\end{array}\right) \quad x_{e}=\left(\begin{array}{l}
0 \\
1
\end{array}\right) \quad x_{\mu}=\left(\begin{array}{l}
\cos ^{1} / 2 \theta_{\mu} \\
\sin ^{1} / 2 \theta_{\mu}
\end{array}\right)
$$

where the electron moves along the $\mathrm{Z}$ axis and the muon spin direction is in the $\mathrm{X}-\mathrm{Z}$ plane, we have for the matrix element

$\mathrm{H}_{\mathrm{f} i}=-4 \mathrm{~g}\left(\cos \frac{1}{2} \theta_{\nu_{\mu}}\right)\left(\cos \frac{1}{2} \theta_{\mu} \sin \frac{1}{2} \theta_{\nu_{e}} \mathrm{e}^{1 \phi_{\nu_{e}}}-\sin \frac{1}{2} \theta_{\mu} \cos \frac{1}{2} \theta_{\nu_{e}}\right)$

and

$$
\left|H_{f 1}\right|^{2}=4 g^{2}\left(1+\cos \theta_{\nu_{\mu}}\right)\left(1-\cos \theta_{\mu} \cos \theta_{\nu_{e}}\right)
$$

af ter averaging over $\phi_{\nu_{e}}$.

We can then use this to calculate the transition probability

$$
P=\frac{2 \pi}{\hbar}\left|H_{f i}\right|^{2} \frac{\mathrm{dN}_{f}}{d E}=\frac{2 \pi}{\hbar} \int\left|H_{f i}\right|^{2} d_{f} \delta\left(E_{f}-E_{i}\right) .
$$

We have

$$
d N_{f}=\frac{E_{e}^{2} d E_{e} d \Omega_{e}}{(2 \pi h c)^{3}} \frac{E_{\nu_{\mu}}^{2} d E_{\nu_{\mu}} d \Omega_{\nu_{\mu}}}{(2 \pi h c)^{3}},
$$


wh1ch gives

$$
\begin{aligned}
P & =\frac{2 \pi}{h} \frac{E_{e} d E_{e}^{2} d \Omega_{e}}{(2 \pi f c)^{6}} \int 2 \pi E_{\nu_{\mu}}^{2} d E_{\nu_{\mu}} d\left(\cos \theta_{\nu_{\mu}}\right) \\
& \times\left|H_{f i}\right|^{2} \delta\left(E_{e}+E_{\nu_{\mu}}+E_{\nu_{e}}-2 E_{\max }\right),
\end{aligned}
$$

where $E_{\max } i s$ the maximum energy of any one particle, $\frac{1}{2} m_{11} c^{2}$.

Evaluation of this expression ylelds

$$
\begin{aligned}
& N\left(E_{e}, \Omega_{e}\right) d E_{e} d \Omega_{e}=P \\
& \quad=\frac{g^{2}}{2 \pi^{4} h^{7} c^{6}} E_{\max } E_{e}^{2}\left[\left(3 E_{\max }-2 E_{e}\right)+\cos \theta_{\mu}\left(E_{\max }-2 E_{e}\right)\right] d E_{e} d \Omega_{e} .
\end{aligned}
$$

Integrating over angles finally yields the desired spectrum shape:

$$
N\left(E_{e}\right) d E_{e}=\frac{g^{2}}{3 \pi^{3} h^{7} c^{6}} E_{\max } E_{e}^{2}\left(3 E_{\max }-2 E_{e}\right) d E_{e},
$$

or, changing variables to $\varepsilon-\frac{E_{e}}{E_{\max }}$,

$$
N(\varepsilon) d \varepsilon=\frac{g^{2}\left(E_{\max }\right)^{5}}{3 \pi^{3} h^{7} c^{6}} \varepsilon^{2}(3-2 \varepsilon) d \varepsilon \text {. }
$$

We can normalize this spectrum to $\int_{0}^{1} N(\varepsilon) d \varepsilon=1$; this yields

$$
\mathrm{N}(\varepsilon) \mathrm{d} \varepsilon=2 \varepsilon^{2}(3-2 \varepsilon) \mathrm{d} \varepsilon \quad \text {. }
$$


As mentlorad above, this gives the $e^{+}$spectrum from $\mu^{+}$decay, since there 18 no dependence on spin, and also the antineutrino spectrum.

In general, without taking into account specific helicities, the energy spectrum of the particles emitced in muon decay is given by

$$
N(\varepsilon) d \varepsilon=4 \varepsilon^{2}\left[3(1-\varepsilon)+\frac{2}{3} \rho(4 \varepsilon-3)\right] d \varepsilon(\operatorname{Ref} \cdot 17),
$$

where $\rho$ is the Michel parameter, which contains all the spin dependence and which can vary between 0 and 1 . Assuming the positron and neutrinos are a11. helicity eigenstates, which is true if the positron mass is neglected, then the positron and antineutrino will have the same energy spectrum, corresponding to $\rho=\frac{3}{4}$, which is that derived above. The neutrino will have a spectrum corresponding to $\rho=0$, since $N$ must vanish at $\varepsilon=1$ in order to conserve spin. The neutrino spectrum is therefore

$$
\mathrm{N}(\varepsilon) \mathrm{d} \varepsilon=12 \varepsilon^{2}(1-\varepsilon)
$$

17. Ibid., p. 214 . 


\section{Inverse Beta Decay}

We now discuss the reaction $\bar{\nu}_{e} p \rightarrow n e^{+} .18$ The transition probability for this reaction is given by

$$
W=\frac{2 \pi}{h}|H|^{2} \frac{d N}{d E}
$$

The cross section is given by the transition rate divided by the Incoming neutrino flux:

$$
\mathrm{d \sigma}=\frac{\mathrm{W}}{\mathrm{c}}=\frac{2 \pi}{\mathrm{ch}}|\mathrm{H}|^{2} \frac{\mathrm{dN}}{\mathrm{dE}}
$$

Taking the coupling constant out of $|\mathrm{H}|^{2}$, and defining $|\mathrm{H}|^{2}=\mathrm{g}^{2}|\mathrm{M}|^{2}$, we have

$$
\mathrm{d} \sigma=\frac{2 \pi}{\mathrm{ch}} \mathrm{g}^{2}|\mathrm{M}|^{2} \frac{\mathrm{dN}}{\mathrm{dE}}
$$

No $\%$,

$$
\frac{\mathrm{dN}}{\mathrm{dE}}=\frac{\mathrm{p}_{\mathrm{e}}^{2} \mathrm{dp} \mathrm{e}}{(2 \pi \zeta)^{3}} \frac{\mathrm{d} \Omega_{\mathrm{e}}}{\mathrm{dE_{e }}}=\frac{\mathrm{m}^{2} \mathrm{c}}{(2 \pi \hbar)^{3}} \varepsilon \sqrt{\varepsilon^{2}-1} \mathrm{~d} \Omega_{\mathrm{e}}
$$

where $\varepsilon=\mathrm{E} / \mathrm{mc}^{2}$, and the mass is the mass of the electron. The recoil of the neutron is neglected here. Integrating over angles yields

18. Ibid., pp - 207-210. 


$$
\sigma=\frac{g^{2} m^{2}}{\pi \hbar^{4}}|M|^{2} \varepsilon \sqrt{\varepsilon^{2}-1}
$$

The matrix element $|M|^{2}$ contains the transitions between inttial and final states, summed over the spins.19 For $E_{\nu} \sim \mathbf{m}_{e} c^{2}$ (1.e., $\varepsilon \sim 1$ ), $|M|^{2} \sim 5.20$

It has, however, been pointed out by $0^{\circ}$ Connel121 that there are energy-dependent weak magnetism terms in $|M|^{2}$, which were neglected by Wu and Moskowski, which become Important at energies of interest for this experiment. He finds for $\sigma$ (where now $h=c=1$ )

$$
\left.\sigma=\frac{G^{2}}{\pi} P_{e^{E}} E^{[l}+3 F_{A}^{2}+\frac{20}{3}\left(\frac{E_{\nu} \mu_{v}}{2 m_{p}}\right)^{2}-16 F_{A}\left(\frac{E_{\nu} \mu_{v}}{2 m_{p}}\right)\right] .
$$

Here, $\mu_{\mathrm{v}}$ is the nucleon vector magnetic moment, $F_{A}$ is the axial vector coupling constant, and $\mathrm{G}=10^{-5} / \mathrm{m}_{\mathrm{p}}^{2}$. We can now takc this, multiplied by the antineutrino energy spectrum given above (for the antineutrino from muon decay), and integrate over the energy to get an average cross section. This yields

$$
\left.<\sigma_{\mathrm{v}_{\mathrm{e}}}\right\rangle=1.69 \times 10^{-40} \mathrm{~cm}^{2}
$$

19. Ibid., p. 48 .

20. Ibld., p. 208.

21. J. S. O'Connel1, Los Alamos Scientific Laboratory report LA-5175-MS, March 1973. 
$0^{\prime}$ Connell also calculates the cross section for neutrinos on deuteriun: 22

$$
\nu_{e} d+p_{p e}^{-}
$$

He considers first the ${ }^{3} S_{1} \rightarrow{ }^{1} S_{0}$ transition, and later adds corrections for higher nucleon partial waves. Using effectlve range theory for the nuclear wave functions, and integrating numerically over the solid angle and the neutrino energy spectrum, he finds for an average cross section

$$
\left.<\sigma_{\nu^{d}}\right\rangle=0.44 \times 10^{-40} \mathrm{~cm}^{2}
$$

He then adds corrections for higher nucleon partlal waves, and finds

$$
<v_{e} d^{>}=0.48 \times 10^{-40} \mathrm{~cm}^{2}
$$

Similar calculations have been done on ${ }^{12} \mathrm{C}$ (Ref. 23) and $15_{n}$ (Ref. 24) with the following results:

$$
\begin{aligned}
& \left\langle\sigma_{\nu_{e}}{ }^{12} \mathrm{c}\right\rangle=0.146 \times 10^{-40} \mathrm{~cm}^{2} \\
& \left\langle\sigma_{\nu_{e}}{ }^{16} 0\right\rangle=0.052 \times 10^{-40} \mathrm{~cm}^{2} .
\end{aligned}
$$

\section{Ibid.}

23. J. S. O'Conne11, T. W. Donnelly, and J. D. Walecka, Phys. Rev. Cㅡ, 719 (1972).

24. T. W. Donnelly, Phys. Lett. 43B, 93 (1973). 
An estinate has also been ande of the reaction 25

$$
v_{e}+{ }^{27} \mathrm{Al}+e^{-}+{ }^{27} \mathrm{SI}_{\mathrm{B}-\mathrm{B} .},
$$

with the result

$$
\left\langle\sigma_{V_{e}} 27 \mathrm{Al}\right\rangle=0.23 \times 10^{-40} \mathrm{ca}^{2}
$$

\section{B. GAUGE THEORIES 26}

\section{An Exarple:}

\section{Quantun Electrodynantes as a U(1) Abel Ian Gauge Theory}

Current ticories of reak Interactions are based on gauge theories. These theories use the symetry structure of certain groups, such as SU(2) or U(1), to model symetries of a Lagrangian. The Heinberg-Salan model, which has so far successfully modelled the weak and electromagnetic interactions, is a gauge theory whose group is SU(2) $\times U(1)$. We will start our discussion of gauge theorles by looking at quantum electrodynamics, which can be described as a gauge theory whose group is $U(1)$. The Heinberg-Salam model contains QED as a subgroup, so this discussion will serve to fatroduce gauge theorles in general and the Helnberg-Salan nodel in particular.

We begin by reviewing the Lagrangian formallsw within the context of gauge theorles. Notation will be as follows:

25. LAMpF Proposal 20, in LAMPF Neutrino Facility Proposal, op. cit. 26. R. W. Brown, lecture notes, sumer 1978 . 


$$
\hbar=c=1
$$

sum over repeated indices

$$
\begin{aligned}
& x^{\mu}=(t,+), x_{y}=(t,-\dot{x}) \\
& g_{\mu \nu}=8^{\mu \nu}=\left(\begin{array}{rrr}
1 & 0 & 0 \\
& -1 & -1
\end{array}\right) \\
& 8_{\mu}^{v}-8_{\mu a} g^{\alpha v}=\left(\begin{array}{llll}
1 & 0 & \\
& \vdots & 1
\end{array}\right) \\
& \partial_{\mu}=\frac{\partial}{\partial x^{\mu}}=\left(\frac{\partial}{\partial}, \vec{\nabla}\right) \quad \partial^{\mu}=\frac{\partial}{\partial x_{\mu}}=\left(\frac{\partial}{\partial t},-\vec{\nabla}\right) .
\end{aligned}
$$

We have, from the invariance of the functional integral of the Lagrangian density L, the Euler-Lagrange equations of motion:

$$
\frac{\partial \underline{L}}{\partial \dot{\phi}}-\partial_{\mu} \frac{\partial \underline{L}}{\partial\left(\partial_{\mu} \phi\right)}=0 \quad \underline{L}=\underline{L}\left(\phi, \partial_{\mu} \phi\right) \text {. }
$$

The free-field equation for a spin $\frac{1}{2}$ particle (the electron), which is the Dirac equation, is then

$$
(i)-m) \downarrow=0 \text {, }
$$

where

$$
\gamma=\partial^{\mu} \gamma_{j} .
$$


Th1s ylelds the Lagrangian density

$$
\underline{L}=\bar{\varphi}(17-m) \psi
$$

We nov discuss continuous symatries of the Lagranglan. If a continuous transformation $\phi^{+} \phi^{\prime}=\mathrm{J} \phi$ laves the Lagranglan unchanged, 1t is referred to as a continuous symetry. We way then use Noether ${ }^{\circ}$ Theorem to find conserved quartities:

If any continuous transformation leaves the Lagrangian invarlant, and ti.e equations of mocion Invariant in form, there exists a corresponding conservation law and a constant of motion.

Now, we need to know how to calculate the conserved currents which correspond to the constants of mation. Let us look at an infinitesimal transf ormation:

$$
\phi(x)+\phi^{\prime}(x)=\phi(x)+\lambda \phi(x),
$$

where $|\lambda| \ll 1$. Then

$$
\begin{aligned}
\frac{d \underline{L}}{d \lambda} & =0=\frac{\partial \underline{L}}{\partial \phi} \frac{\partial \phi}{\partial \lambda}+\frac{\partial \underline{L}}{\partial\left(\partial_{\mu} \phi\right)} \frac{\partial\left(\partial_{\mu} \phi\right)}{\partial \lambda} \\
& =\partial_{\mu}\left[\frac{\partial \underline{L}}{\partial\left(\partial_{\mu} \phi\right)} \phi\right] .
\end{aligned}
$$


This 18 an equation of the form $\partial_{\mu} \mathrm{J}^{\mu}=0$; we may therefore treat $\mathrm{J}^{\mu}$ as a conserveó current. More generally, to include transformations among a set of $\phi_{1}$ :

$$
\phi_{1}+\phi_{1}^{\prime}=\phi_{1}+\lambda T_{i j} \phi_{j}
$$

Then,

$$
J^{\mu}=\frac{\partial L}{\partial\left(\partial_{\mu} \phi_{1}\right)} T_{1 j} \phi_{j} \text {. }
$$

Now, If the surface cerms vanish or cancel (these are the same arguments used in classical electrodynamics), then

$$
\int \vec{\nabla} \cdot \vec{J}_{d}^{3} x=0
$$

or,

$$
\int d^{3} x \partial_{\mu} J^{\mu}=\frac{d}{d t} Q=0
$$

where $Q=\int d^{3} x J^{0}$, a conserved charge. Slince $d Q / d t=0$, then $[Q, H]=0$ and there exist simultaneous eigenfunctions of $Q$ and $H$, leading to conserved quantum numbers. Also, $Q$ is the generator of the transformation, in the following sense: If $\phi^{+} \phi^{\prime}=U \phi$ where $U=e^{i \Lambda}, U$ $=1+1 \Lambda$ if $\Lambda$ is infinitesimal (i $<\lambda$ above); then $\delta \phi=1 \Lambda \phi$. The generator $G$ of a transformation is defined by

$$
\delta \phi=1 \Lambda[G, \phi] ;
$$


therefore

$$
\phi^{\circ}=e^{I \Lambda G} \phi e^{-i \Lambda G} .
$$

Bữ since

$$
Q=1 \int d^{3} x \frac{\partial \underline{L}}{\partial \dot{\phi}} \phi \text { (since } T_{i f}=1 \delta_{1\}} \text { here) }
$$

and

$$
\frac{\partial \underline{L}}{\partial \dot{\phi}}=\pi,
$$

where

$$
\{\pi, \phi\}=1 \delta^{3}\left(\vec{x}-\vec{x}^{\prime}\right),
$$

(we can choose our commutation relations to be at equal time if $\mathrm{dQ} / \mathrm{dt}=0$ ), we have

$$
\begin{aligned}
{[Q, \phi] } & =1 \int d^{3} x(\pi\{\phi, \phi\}-\{\pi, \phi\} \phi) \\
& =\phi ;
\end{aligned}
$$

therefore $Q=G$. 
Now we can discugs global gauge tnveriance. A phase change for constant $\theta$

$$
\phi+\phi^{\prime}=e^{1 \theta} \phi
$$

Io a global gauge transformat Lon; if the Lagrangian is invariant under this transformation, it is a flobal symmetry. Then

$$
\phi_{1}+e^{1 \theta Q_{\phi_{1}}} \mathrm{e}^{-1 \theta Q}=\mathrm{e}^{1 q_{1} \theta_{\phi_{1}}}
$$

where $q_{1}$ is the elgenvalue of $Q$ corresponding to $\phi_{1}$. Therefore

$$
e^{1 \theta Q_{L} e^{-19 Q}}=L
$$

If $\sum_{i} q_{1}=0$ for each term -- this is effectively sharge conservation. Since $e^{1 \theta}$ is unttary (if we call $U=e^{19}$, then $U^{-1}=U^{0}$ ) and abelian (the elements of the set commute), it is a representation of the group $\mathrm{U}(1)$.

Specifically, in the case of the electron:

$$
\underline{L}=\bar{\psi}(1 \not-m) \psi
$$

(as above); then the gauge transformation

$$
\psi \rightarrow e^{1 e \theta} \psi ;
$$

since this is a $\mathrm{U}(1)$ global symmetry, $J_{\mu}=\bar{\psi} \gamma_{\mu} \psi$ is conserved. Then: 


$$
\begin{aligned}
z & =-\frac{1}{4} F^{\mu \nu F_{\mu \nu}}+\bar{\psi}(1 \eta+e A-m) \psi \\
F_{\mu \nu} & =\partial_{\mu} A_{\nu}-\partial_{\nu} A_{\mu}
\end{aligned}
$$

with the equations of motion

$$
\begin{aligned}
\partial_{\mu} F^{\mu \nu}= & e J^{\nu} \\
& (1 \nu+e \alpha-m) \psi=0 .
\end{aligned}
$$

These are Invariant under

$$
\begin{gathered}
\psi+e^{1 \theta(x)} \psi \\
A_{\mu}+A_{\mu}+\frac{1}{e} \partial_{\mu} \theta
\end{gathered}
$$

These, then, are the equations of quantum electrodynamics, with $A_{\mu}$ the four-vector potential.

\section{The Weinberg-Salam ModeI}

We can now discuss the Welnberg-Salam model. The Weinberg-Salam model is an SU(2) $\times U(1)$ gauge theory which unifies electromagnetic and weak Interacions. The discussion wili begin with massless leptons, and with just the electron and its neutrino. The muon and its neutrino will be added later. The free-fleld Lagranglan density is

$$
\underline{L}=\bar{v} 1 \partial \nu+\overline{\mathrm{e}} 1 \gamma_{\mathrm{e}},
$$


where $v$ and $e$ are the flelds of the neutrino and electron. Since the neutrino is observed to be in the negative hellcity state exclusively,

$$
v=v_{L}=\frac{1-\gamma_{5}}{2} v
$$

But the electron can be In either hellcity state:

$$
e=\left(\frac{\left(1+\gamma_{5}\right)}{2}+\frac{\left(1-\gamma_{5}\right)}{2}\right) e=e_{R}+e_{L} \text {. }
$$

Now,

$$
\underline{L}=\bar{\nu}_{L} 1 \not \nu_{L}+\bar{e}_{L} 1 \gamma e_{L}+\bar{e}_{R} 1 \not e_{R}
$$

since

$$
\bar{e}_{R} \mathcal{I} \mathrm{e}_{\mathrm{L}}=\overline{\mathrm{e}}_{\mathrm{L}} \mathcal{1} \boldsymbol{\gamma} \mathrm{e}_{\mathrm{R}}=0
$$

This demonstrates that the two helicity states do not $\mathrm{mlx}$ in the massless case.

We put the electron and neutrino into a doublet; this will allow for e-v interactions $v i a W^{ \pm}$exchange. This gives a left-handed doublet and a right-handed singlet:

$$
L=\left(\begin{array}{l}
\nu_{L} \\
e_{L}
\end{array}\right) ; R=e_{R}
$$

We now have the transformation

$$
L \rightarrow U L=e^{i \vec{\theta} \cdot \vec{\sigma} / 2} L ; R+R
$$


This is a local symmetry of su(2) (because of $\vec{\sigma}$ ), and ylelds

$$
\underline{L}=-\frac{1}{4} \vec{F}_{\mu \nu} \cdot \vec{F} U \nu+\bar{L} 1\left(z-1 g \frac{\vec{\sigma}}{2} \cdot \vec{L}\right) L+\bar{R} 1 \eta_{R}
$$

where

$$
\vec{F}_{\mu \nu}=\partial_{\mu} \vec{A}_{\nu}-\partial_{\nu} \vec{A}_{\mu}+\overrightarrow{g A}_{\mu} \times \vec{A}_{\nu} .
$$

We examine the interaction term more closely:

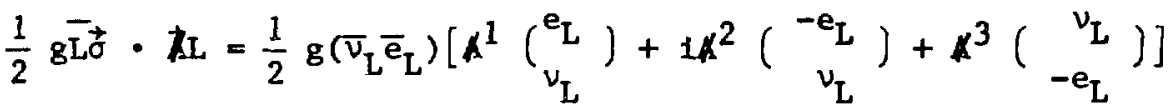

$$
\begin{aligned}
& =\frac{1}{2} g\left[\bar{v}_{L}\left(A^{1}-1 \AA^{2}\right) e_{L}+\bar{e}_{L}\left(A^{1}+1 \not^{2}\right) v_{L}+\bar{v}_{L} \Lambda^{3} v_{L}-\bar{e}_{L} A^{3} e_{L}\right] .
\end{aligned}
$$

We see that interactions between $\nu$ and e are mediated by $\frac{1}{\sqrt{2}}\left(A_{\mu}^{1}+\right.$ $\left.1 A_{\mu}^{2}\right)=W_{\mu}$; then $W_{\mu}^{+}=\frac{1}{\sqrt{2}}\left(A_{\mu}^{1}-1 A_{\mu}^{2}\right)$. The action of the $W$ fleld 1 s to create $\mathrm{W}^{+}$intermediate vector bosons, and to destroy $\mathrm{W}^{-}$. In explicit V-A form,

$$
\frac{\sqrt{2}}{2} g \bar{v}_{L} H^{+} e_{L}=\frac{1}{\sqrt{2}} g \frac{1}{2} \bar{v} \gamma_{\mu}\left(1-\gamma_{5}\right) \mathrm{eW}^{\mu+},
$$

where we can define

$$
\mathrm{g}_{\mathrm{W}}=-\frac{1}{\sqrt{2}} \mathrm{~g} .
$$


$-28-$

Note that $A^{3}$ cannot represent the photon field, since it mediates $v i v$. coupling, and since it interacts only with $e_{L}$ and not with $e_{R}$. We st111 have a $U(1)$ symmetry to gauge; for this we can define a "weak hypercharge", $Y=N_{R}+\frac{1}{2} N_{L}$, where $N_{R}$ and $N_{L}$ are the right- and left-handed lepton numbers. In analogy with strong interactions, $Q=T_{3}$ - $Y$, where $Q 18$ the charge and $T_{3} 1 s$ the third component of the weak 1sospin. $\mathrm{T}_{3}$ can be found from the doublet and singlet assignments of $\nu_{L}, e_{L}$, and $e_{R}$. We can now gauge the hypercharge:

$$
L \rightarrow e^{1 \theta / 2} L ; R \rightarrow e^{1 \theta_{R}},
$$

since $Y=\frac{1}{2}$ for $L$ and $Y=1$ for $R$. Then

$$
\begin{aligned}
& \mathrm{L}=-\frac{1}{4} \overline{\mathrm{F}}_{\mu \nu} \cdot \mathrm{F}^{\mu \nu}-\frac{1}{4} \mathrm{~B}_{\mu \nu} \mathrm{B}^{\mu \nu}+\overline{\mathrm{L}} 1\left(\mathrm{~g}-1 \mathrm{~g} \frac{\vec{\sigma}}{2} \cdot \vec{\not}-1 \frac{1}{2} \mathrm{~g}^{\circ} \not \mathrm{g}\right) \mathrm{L} \\
& +\bar{R} 1\left(\not{\theta}-1 \mathbf{g}^{\prime} \not{b}\right) \mathrm{R},
\end{aligned}
$$

where

$$
\mathrm{B}_{\mu \nu}=\partial_{\mu} \mathrm{B}_{\nu}-\partial_{\nu} \mathrm{B}_{\mu}
$$

The Interaction term ts

$$
\frac{1}{2 \sqrt{2}} g\left[\bar{\nu}_{\mu}\left(1-Y_{5}\right) e W^{\mu+}+h \cdot c \cdot\right]+\frac{1}{2} \bar{\nu}_{L}\left[g A^{3}+g^{\prime} B\right] \nu_{L}
$$

$$
+\frac{1}{2} \bar{e}_{L}\left[-g A^{3}+g^{\prime} \not\right] e_{L}+g^{\prime} \bar{e}_{R} \not e_{R}
$$


We redefine the fieldo $A_{3}, B \rightarrow Z$, $A$ :

$$
\begin{aligned}
& z_{\mu}=\frac{1}{\sqrt{g^{2}+g^{-2}}}\left(8 A_{\mu}^{3}+g^{\prime} B_{\mu}\right) \\
& A_{\mu}=\frac{1}{\sqrt{g^{2}+g^{-2}}}\left(-g^{\prime} A_{\mu}^{3}+g^{B} B_{\mu}\right),
\end{aligned}
$$

so the neutral current part of $I$ is

$$
\begin{gathered}
\frac{1}{2} \sqrt{g^{2}+g^{-2}} \bar{\nu}_{L} z \nu_{L}+\frac{1}{\sqrt{g^{2}+g^{\prime 2}}}\left[\frac{g^{\prime 2}-g^{2}}{2} \bar{e}_{L} z e_{L}+g^{\prime 2} e_{R} z e_{R}\right] \\
+\frac{g g^{\prime}}{\sqrt{g^{2}+g^{\prime 2}}}\left[\bar{e}_{L} A e_{L}+\bar{e}_{R} A e_{R}\right]
\end{gathered}
$$

If we define $e=8 g^{\circ} /\left(g^{2}+g^{-2}\right)^{1 / 2}$, A is the same as the $A$ in quantum electrodynamics (above), and we have recovered the electromagnetic interaction, with no couplings to the neutrinos.

Because the transformation of the fields $A_{3}, B+z$, A preserves the normalization, it can be expressed in terms of an angle:

$$
\left(\begin{array}{l}
z_{\mu} \\
A_{\mu}
\end{array}\right)=\left(\begin{array}{cc}
\cos \theta_{W} & \sin \theta_{W} \\
-\sin \theta_{W} & \cos \theta_{W}
\end{array}\right)\left(\begin{array}{l}
A_{\mu}{ }^{3} \\
B_{\mu}
\end{array}\right),
$$

where $\theta_{\mathrm{W}}$ is the Welnberg angle. In terms of 8 and $8^{\circ}$, above: 
$\cos \theta_{W}=\frac{g}{\sqrt{g^{2}+g^{\prime 2}}} ; \sin \theta_{W}=\frac{g^{\prime}}{\sqrt{g^{2}+g^{\prime 2}}} ; \tan \theta_{W}=g^{\prime} / g$,

and

$$
e=g \sin \theta_{W}
$$

Finally, the massless Weinberg-Salam model for $e, \nu_{e}$ can be written

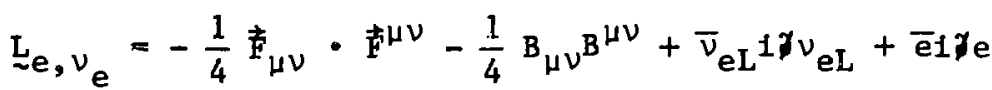

$$
\begin{aligned}
& +\frac{e}{2 \sqrt{2} \sin \theta_{W}}\left[\bar{\nu}_{e} \gamma_{\mu}\left(1-\gamma_{5}\right) e W^{\mu+}+\bar{e}_{\mu}\left(1-\gamma_{5}\right) \nu_{e} W^{\mu}\right] \\
& +e \bar{e} \gamma_{\mu} e A^{\mu}+\frac{e}{2 \sin 2 \theta_{W}}\left[\bar{\nu}_{e} \gamma_{\mu}\left(1-\gamma_{5}\right) \nu_{e}\right. \\
& \left.+\bar{e} \gamma_{\mu}\left(4 \sin ^{2} \theta_{W}-1+\gamma_{5}\right) e\right] z
\end{aligned}
$$

(care should be taken not to confuse the $e$ which is the charge with the e which is the electron fle1d). The muon and its neutrino can be added simply by Inserting extra terms of exactly the same form as the terms above involving $\nu_{e}$ and $e$, but which involve the fields $\nu_{\mu}$ aind $\mu$ instead. Then 


$$
\begin{aligned}
\underline{L}_{\text {tot }}= & \underline{L e}_{e, \nu_{e}}+\bar{\nu}_{\mu \mathrm{L}} 1 \gamma \nu_{\mu L}+\bar{\mu} 1 \gamma_{\mu} \\
& +\frac{e}{2 \sqrt{2} \sin \theta_{W}}\left[\bar{\nu}_{\mu} \gamma_{\mu}\left(1-\gamma_{5}\right) \mu W^{\mu+}+\bar{\mu} \gamma_{\mu}\left(1-\gamma_{5}\right) \nu_{\mu} W^{\mu}\right]+e \bar{\mu} \gamma_{\mu} \mu \Lambda^{\mu} \\
& +\frac{e}{2 \sin 2 \theta_{W}}\left[\bar{\nu}_{\mu} \gamma_{\mu}\left(1-\gamma_{5}\right) \nu_{\mu}+\bar{\mu} \gamma_{\mu}\left(4 \sin ^{2} \theta_{W}-1+\gamma_{5}\right) \mu\right] z
\end{aligned}
$$

The parameters $\sin \theta_{W}$ and the flelds $W^{\mu}$ and $Z$ are taken to be the same for muons and electrons, in order to preserve $\mu-e$ universality.

Masses in the theory arise from spontaneous symmetry breaking. By Inserting mass terms in the Lagrangian -- these take the form of Interactions between $e_{L}$ and $e_{R}$, since a massive electron is not an elgenstate of helicity -- it is possible to derive masses for the $W$ and $z$ flelds in terms of the Weinberg angle.

The model in this form does not have alectron and muon flelds mixing in any way, 1.e. there is no interaction written down involving both e and $\mu$. This leads to an additive muon conservaton number. However, the additive number does not correspond to any kind of gauge Invariance; the only gauge symmetries are charge conservation and rightand left-handed lepton number conservation (helicity conservation). This means that if interactions which violated an additive muon number were observed, as in the decay $\mu \rightarrow e \gamma$, such an interaction term could be added. This involves putting $e$ and $\mu$ in the same multiplet. There also has been no mention of the tau lepton. 27 It can be added in exactly the 
save maner as the muon and its neutrino, and this procedure clearly generalizes to any number of leptons, each with its own neutrino. A generalization of this theory which leads to a aultiplicatively conserved lepton number will be discussed below.

C. THEORETICAL CONSIDERATIONS OF $\bar{y}_{e}$ FROM $\mu+$ DECAY

\section{Multiplicative Law}

The need for a muon number conservation law in addition to a lepton number congervation law was flrst suggegted by the absence of neutrinoless $\mu-e$ transitions, such as $\mu \rightarrow e \gamma, \mu \rightarrow 3 e$, and $\mu Z+Z^{\prime}$. Current 1imits on these transitions are as follows:

$$
\begin{aligned}
& \mu^{-}+e^{-} e^{-} e^{+}<1.9 \times 10^{-9}(\text { Ref } \cdot 28) \\
& \mu^{-} \text {er }<1.9 \times 10^{-10}(\text { Ref } \cdot 29) \\
& \mu z \rightarrow e z^{\circ}<4 \times 10^{-10} \text { (Ref. 30) }
\end{aligned}
$$

These processes are allowed by an additive lepton number congervation law -- one which assigns lepton number as Indicated in Table $I$ and then demands that the total number of leptons be conserved. An additive law of muon number conservation can be formulated in a corresponding way, with quantum number assignments as in Table II. This does not replace lepton number conservation, but supplements ft. This conservation law requires the existence of two neutrinos, one with nonzero muon number.

28. S. M. Korenchenko et al., Soviet Physics JETP 43, 1 (1976). 29. J. D. Bowman et al., Phys. Rev. Lett. 42, 556 (1979).

30. A. Badertscher et al., Phys. Rev- Lett. 39, 1385 (1977). 
TABLE I

LEPTON NUTBER ASS IGATEN

Particle

$e^{-}, \mu^{-}, v$

$e^{+}, \mu^{+}, \bar{v}$

al1 others
Lepton Nuber

$+1$

$-1$

0

TABLE II

MUON NUMBER ASS IGNMENT

\section{Particle}

$\mu^{-}, v_{\mu}$

$\mu^{+}, \bar{v}_{\mu}$

all others
Yuon Number

$+1$

$-1$

3

An alternative formulation of the additive law would be to have electron and muon number conserved separately; this avtomatically conserves total lepton number, which is the sum of electron and muon numbers.

Because all the unobserved reactions Ilsted above involve odd numbers of muons and electrons, it is possible to forbid them with a multiplicative conservation law as wel1.31 This law involves a suon "parity", assigned agaln as in Table II, but which is conserved in the followling manner:

31. G. Felnberg and S. Weinberg, Phys. Rev. Lett. 6, 381 (1961). 


$$
\pi(-1)^{L_{\mu}}=\text { constant }
$$

(this discussion concerns itself only with the wuon and electron; generalizations which include the tau will be considered later.) Th1s law allows all reactlons allowed by the additive law, forblds the reactions listed above, but allows reactions involving even numbers of muons and electrons which are forbidden by the additive law, such as

$$
\mu^{+}+e^{+} \bar{\nu}_{e^{\nu}}, \mu^{+} e^{--}+\mu^{-} e^{+}, \quad \text { and } e^{-} e^{-}+\mu^{-} \mu^{-} \text {. }
$$

A multiplicative conservation law can be a consequence of $\mu-e$ tontversality. If the unfversality derives from a permutation symetry of two primitive leptons $e^{\circ}$ and $\mu^{\circ}$, which can make transtitions $e^{\prime}-\mu^{\prime}$, the observed particles will not be $e^{\prime}$ and $\mu^{\prime}$ but $e=\left(e^{\prime}+\mu^{\prime}\right) / \sqrt{2}$ and $\mu$ $=\left(\mu^{\prime}-e^{\prime}\right) / \sqrt{2}$. The transition $e^{\prime} \rightarrow \mu^{\prime}$ then corresponds to $e \rightarrow e$ and $\mu$ $\rightarrow-\mu$, which leads to a muon "parity" which is multiplicatively conserved as described above. In the absence of a spectflc model, no coupling constant for the decays allowed only by the multiplicative law can be assumed. It has been shown by Cabibbo and Gatto 32 that this formulation leads tc two neutrinos whtch obey the same conservation law.

Other forms of lepton conservation laws have also been proposed. One, ${ }^{\prime}$ g Konoplneikt and Mahmoud ${ }^{33}$ assigns $L=+1$ to $e^{-}, \mu^{+}, \nu_{e}$, and $\bar{\nu}_{\mu}$, and $L=-1$ to $e^{+}, \mu^{-}, \bar{\nu}_{e}$, and $\nu_{\mu}$. This forbids reactions of the form $\mu^{+} e^{-}+\mu^{-} e^{+}$and $\mu^{-} z+e^{-} Z^{\prime}$, but allows $\mu^{-} Z+e^{+}(Z-2)$ and $k^{+}+\pi^{-} e^{+} \mu^{+}$, although these reactions are forbldden dynamically. The rate expected

32. N. Cabibbo and R. Gatto, Phys. Rev. Lett. 5, 114 (1960).

33. Konopinakt and Mahmoud, Phys. Rev. 92, 1045 (1953). 
in this wodel for these tateractions has been calculated by Prinkoff and Rosen ${ }^{34}$ who find

$$
R\left[\frac{\mu^{-} z+e^{+}(z-2)}{\mu^{-} z+\nu_{\mu}(z-1)}\right]=R\left[\frac{R^{+}+e^{+} \mu^{+} \pi^{-}}{R^{+}+\mu^{+} \nu_{\mu} \pi^{0}}\right] \leqslant 3.2 \times 10^{-14} .
$$

Present experimental limits for $\mathrm{H}^{-}$to $\mathrm{e}^{+}$conversion on a nucleus sre $R<$ $1.5 \times 10^{-9}, 35$ and for the kaon decay, $R=1.4 \times 10^{-8} .36$ Pontecorvo $^{37}$ In a general survey considers all the aborl formulations as vell as one where $L=+1$ for $e^{-}$and $\nu_{e}$ and +2 for $\mu^{-}$and $\nu_{\mu}$; this 18 Indistinguishable from the additive law if there are only two leptons. It is not obvious how to incorporate the $T$ here.

A recent paper by Derman ${ }^{38}$ extends the permutation symetry 1dea above to include the tau and its aeutr1no, and explores the consequences of this symmetry in terms of the Weinberg-Salam model. This symetry leads to the existence of Higgs bosons which carry lepton flavor. Derman shows that in order to have an $a-\mu-\tau$ mass watrix which is completely nondegenerate, one is forced to a unique selection of a vacuum state. This vacuum preserves a two-fold permutation symetry, which leads to a multiplicatively conserved cuantum number, $\pi$, where $\pi=$ \pm 1 . This number is aifferent for $e$ and $\mu$, but the $\tau$ mist have the same quantum number as elther $e$ or $\mu$; the model therefore has important

34. H. Primakoff and S. P. Roser, Phys. Rev. D5, 1784 (1972).

35. A. Badertscher et al. Phys. Lett. $19 \mathrm{~B}, 371$ (1978).

36. E. W. Beter et al., Phys. Rev. Lett. 29, 678 (1972).

37. B. Pontecorvo, Soviet Phys. JETP 26, 984 (1968).

38. Emanuel Derran, Phys. Rev. D19, 317 (1979). 
consequences for $\tau$ decay sodes. The following is a compilation of some of the experimental concequences of Derman's theory, using his aasigneent $\pi_{\tau}=\pi_{\mu}--\pi_{e}$ (The other possibilitg $18 \pi_{\tau}=\pi_{e}=-\pi_{\mu}$ this reaults in the replacenent of $\mu$ by $e$ in the $\tau$ decays.)

$\tau+\mu \gamma:$ branching rat1o $\sim 4 \times 10^{-4}$ assuming $\mathrm{K}_{\mathrm{H} 1 \mathrm{ggs}} \sim 5 \mathrm{GeV}$. Lower 11mit: $>2 \times 10^{-9}$ since at least one H1gge boson must have mass $<250 \mathrm{GeV}$

$\tau+3 \mu$ : branching ratio $\sim 7 \times 10^{-4}$, but could be wuch smaller if al1 $\pi=1$ H1gg8 bosons are very heavy (unlike $\tau+\mu \mathrm{Y}, \tau+$ $3 \mu$ goes through $\pi *+1$ boson exchange only) $\tau+$ Hee: branching ratio $\sim 7 \times 10^{-4}$. Lower $11 m i t: 210^{-10}$. Similar to $T \rightarrow \mu \gamma$.

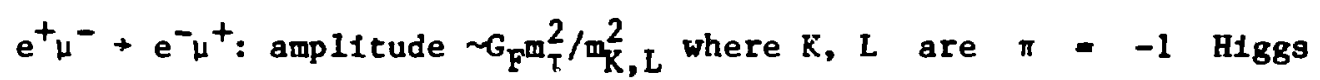
bosons. Present upper $11 \mathrm{mlt}$ on this $1 \mathrm{~s}<5800 \mathrm{G}_{\mathrm{F}} \cdot{ }^{39}$ $\mu^{+} \rightarrow e^{+} \bar{\nu}_{e} \nu_{\mu}:$ amplitude $\sim G_{F} m_{\tau}^{2} / m_{H}^{2}$ where $H$ is a $\pi=-1$ Higgs boson. For $m_{H} \sim 5 \mathrm{GeV}$, amplitude $\sim 0.13 \mathrm{G}_{\mathrm{F}}$, branching rat10 $\sim 2 \%$.

Derman extends this model to the quark sector, which has consequences for $b$ quark decay and for the Higgs boson masses. Since the $\pi=+1$ bosons mediate the Interactions $\pi^{+} \rightarrow \mathrm{e}^{+} \nu_{e}, \mathrm{k}^{\circ} \rightarrow \overline{\mathrm{k}}^{\circ}$, and $\mathrm{k}^{\circ} \rightarrow$ $\mathrm{e}^{+} \mathrm{e}^{-}$, their masses must be large to be consistent with the experimentally observed rates of these processes. This means that these masses must be at least several hundred GeV. No such limit exists, however, on the masses of the $\pi=-1$ bosons, since the interactions they mediate are not subject to very stringent experimental 1imits. Note that this model is valld for the three-lepton case only. If more heavy

39. R. Amato et a1., Phys. Rev. Lett. 21, 1709 (1968). 
leptons are ilscovered, the model would have to be completely reformulated.

\section{Neutrino Osctilations and Decay}

In the presence of a muon number violating interaction and different $v_{e}$ and $v_{\mu}$ uasses, it is possible to have neutrino oscillations and muon neutrino decay (assuming $m_{v_{\mu}}>m_{v}$ ). Decay could occur via the reaction $v_{\mu} \rightarrow v_{e} \gamma$, and w11l be discussed below. Oscillations could occur if the physical neutrinos $v_{e}$ and $v_{\mu}$ are superpositions of two fundamental neutrinos $v_{1}$ and $v_{2}$ with definite masses $m_{1}$ and $m_{2}$. Then $v_{e}$ and $\nu_{\mu}$ are not stationary states and do not have definite masses. To be specific about the formulation of the mixing, $\nu_{e}=\cos \theta \nu_{1}+\sin \theta v_{2}$ and $v_{\mu}=-\sin \theta v_{1}+\cos \theta v_{2}$. The angle $\theta$ describes the strength of the mixing; the oscillation length will depend on $\theta$ and on $\left|m_{1}-m_{2}\right|$. Note that this may superficially resemble the $e^{\prime}-\mu^{\prime}$ mixing described at the beginning of this section. The difference is that $e^{\prime}$ and $\mu^{\prime}$ were assumed to have identical masses, because of universality. This led to a strict separation between the physical $e$ and $\mu$, sther than the mixing one gets with different masses.

The theory of neutrino oscillations will. now be formulated in more deta11.40 The state vectors of the electron and muon neutrinos are $\left|\nu_{e}\right\rangle$ and $\left|v_{\mu}\right\rangle$; of the fundamental neutrinos $\left|v_{1}\right\rangle$ and $\left|v_{2}\right\rangle_{*}$. Then

$$
\left|\nu_{\ell}\right\rangle=\sum_{\sigma=1,2} U_{\ell \sigma}\left|\nu_{\sigma}\right\rangle \quad \ell=e, \mu,
$$

where

40. S. M. B1lenky and B. Pontecorvo, Phys. Reports $4 \underline{4}, 225$ (1978). 
$-38-$

$$
U_{\ell, \sigma}=\left(\begin{array}{cc}
\cos \theta & \sin \theta \\
-\sin \theta & \cos \theta
\end{array}\right)
$$

Since $\left|\nu_{\sigma}\right\rangle$ are the elgenstates of the Handitontan,

$$
\mathrm{H}\left|\nu_{\sigma}\right\rangle=\mathrm{E}_{\sigma}\left|\nu_{\sigma}\right\rangle
$$

A beam of neutrinos created at the $t=0$ will be pure $\left[\nu_{\ell}>(l\right.$ as $e, \mu)$.

At tIme $t$ the state vector of the beam will be

$$
\begin{aligned}
& \left|v_{\ell}\right\rangle_{t}=e^{-1 H t}\left|v_{\ell}\right\rangle=\sum_{\sigma=1,2} v_{\ell \sigma} e^{-1 E_{\sigma} \varepsilon^{2}}\left|v_{\sigma}\right\rangle \\
& \left.=\sum_{\ell^{\prime}=e, \mu} a_{\ell^{\prime}, v_{\ell}}(t) \mid v_{\ell}{ }^{\circ}\right)
\end{aligned}
$$

where

$$
a_{v_{\ell}, v_{\ell}}(t)=\sum_{\sigma=1,2} U_{\ell \sigma^{e}}{ }^{-1 E_{\sigma} t} U_{\ell^{*} \sigma} .
$$

The transition probability is then

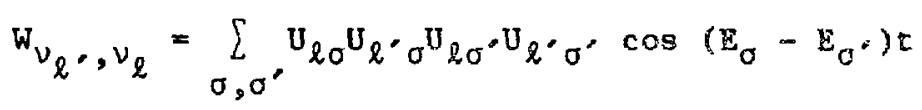

If $\mathrm{p}_{v} \gg \mathrm{m}_{1}, \mathrm{~m}_{2}$, then

$$
\mathrm{E}_{1}-\mathrm{E}_{2} \simeq \frac{\mathrm{m}_{1}^{2}-\mathrm{m}_{2}^{2}}{2 \mathrm{~F}_{v}}
$$

Then 


$$
\begin{aligned}
& W_{v_{\ell, v_{\ell}}}=1-\frac{1}{2} \sin ^{2} 2 \theta\left(1-\cos \frac{2 \pi R}{L}\right) \\
& W_{v_{\ell, \nu^{+}}}=\frac{1}{2} \sin ^{2} 2 \theta\left(1-\cos \frac{2 \pi R}{L}\right) ; \ell+\ell^{\prime},
\end{aligned}
$$

which is the probability of finding $\nu_{\ell}$, at a distance $R$ from a sousce of $v_{\ell}$; the oscillation length $\mathrm{L}$ Is defined as follows:

$$
L=\frac{4 \pi p_{\nu}}{\left|m_{1}^{2}-m_{2}^{2}\right|}
$$

Application to the present experiment requires averaging over the finite detector size and the neutrino energy spectrum; this will be discussed with the results.

The subject of neutrino decay is also discussed by Bilenky and Pontecorvo; ${ }^{41}$ they derive for the probability of the decay $v_{1}+v_{2} \gamma$ the expression

$$
\Gamma\left(\nu_{1}+\nu_{2} \gamma\right)=\frac{9}{16} \frac{G^{2} \alpha}{128 \pi^{4}} m_{1}^{5} \sin ^{2} \theta \cos ^{2} \theta\left(M_{\mu}^{2} / M_{w}^{2}\right)^{2}
$$

Using the extreme values $\theta=\frac{1}{4}, m_{1}=1 \mathrm{MeV}$, evaluation of this expression yields a lifetime in the $\nu_{1}$ rest frame of $\tau_{1} \cong 5 \times 10^{11}$ years. Neutrinos of momentum 20 to $60 \mathrm{MeV} / \mathrm{c}$, as in this experiment, will have $B \cong 1$; the time dilation effect in the lab will make the apparent lifetime even longer. From this we conclude that neutrino decay may be neglected here.

41. Ibid., p. 238 . 
CHAPTER 2

\section{EXPERIMENTAL APPARATUS}

\section{A. CERERKOV COUNTER}

We begin with a brief discussion of Cerenkov radiation. 42 A charged particle moving with constant velocity through a matr $=1$ al can radiate if its velocity is highe: than the velocity of light in the medium. The radiaticn will be emitted at an angle given by

$$
\cos \theta_{c}=c^{\prime} / v
$$

where $c^{\prime}$ is the velocity of light in the medium. It can then be shown that the total energy radiated per unit frequency interval per unit path length by a particle will be

$$
\frac{d I(\omega)}{d x}=\frac{e^{2} \omega}{c^{2}}\left(1-\frac{1}{\beta^{2} \varepsilon}\right),
$$

where $\beta=v / c$ and $\varepsilon$ is the dielectric constant, assumed to be a function of w. Assuming that $\varepsilon$ is constant through the visible and near-ultraviolet regions, which are of interest here, the amount of radiation is directly proportional to $\omega$. Also, since $d I / d x$ is not a function of $x$, the amount of light emitted will depend on the distance New York, 1962, pp. 494-499. 
traveled. If the size of the detector ls large compared to the range of the particle, the total amount of light will be proportional to the energy of the particle, assuming that $\beta$ stays coristant. In our case, we wish to measure electron energtes of 20 to $60 \mathrm{MeV}$; $\beta$ for electrons will be constant down to energies of the order of $1 \mathrm{MeV}$, so this is a good assumption.

The Cerenkov counter, 1llustrated in $\mathrm{Fig}$. 1, was a cube $1.8 \mathrm{~m}$ on a side, with cast epoxy talls. Nonmetal construction was chosen to minimize electron-neutrino interactions in the walls, since electron-neutrino cross sections are known to be small on carbon and oxygen (as detalled in the theoretical section). The cubical shape, while it was not ideal hydrostatically, maximized the active water volume inside a box of flat scintillators used as an active cosmic-ray shield.

The Cerenkov counter was fabricated by the Formcrete Company. 43 The walls were made from EPON 828 resin, 44 with curing agent $z^{45}$ and $10 \%$ woodflour filler, and were $1.3-\mathrm{cm}$ thick. Each wall was cast Independently, then cured at $65^{\circ} \mathrm{C}$ for 14 hours. Five sides of the counter were then assembled Inside an aluminum L-bar frame, iiiustrated in Fig. 2, with 3-cm diagonal epoxy filling (EPON 872,46 curing agent $\mathrm{Z}$ ) completing the cube. The top of the counter was a removable lid bolted down over a rubber gasket; it was otherwise Identical to the other sides.

43. Formcrete Co., P. 0. Box 1153, Manchester, CT 06040.

44. Shell Chemfcal Co., One Shell Plaza, P. 0. Box 2463, Houston, TX 77001 .

45. Shell Chemical Co., as above.

46. Shell Chemical Co., as above. 


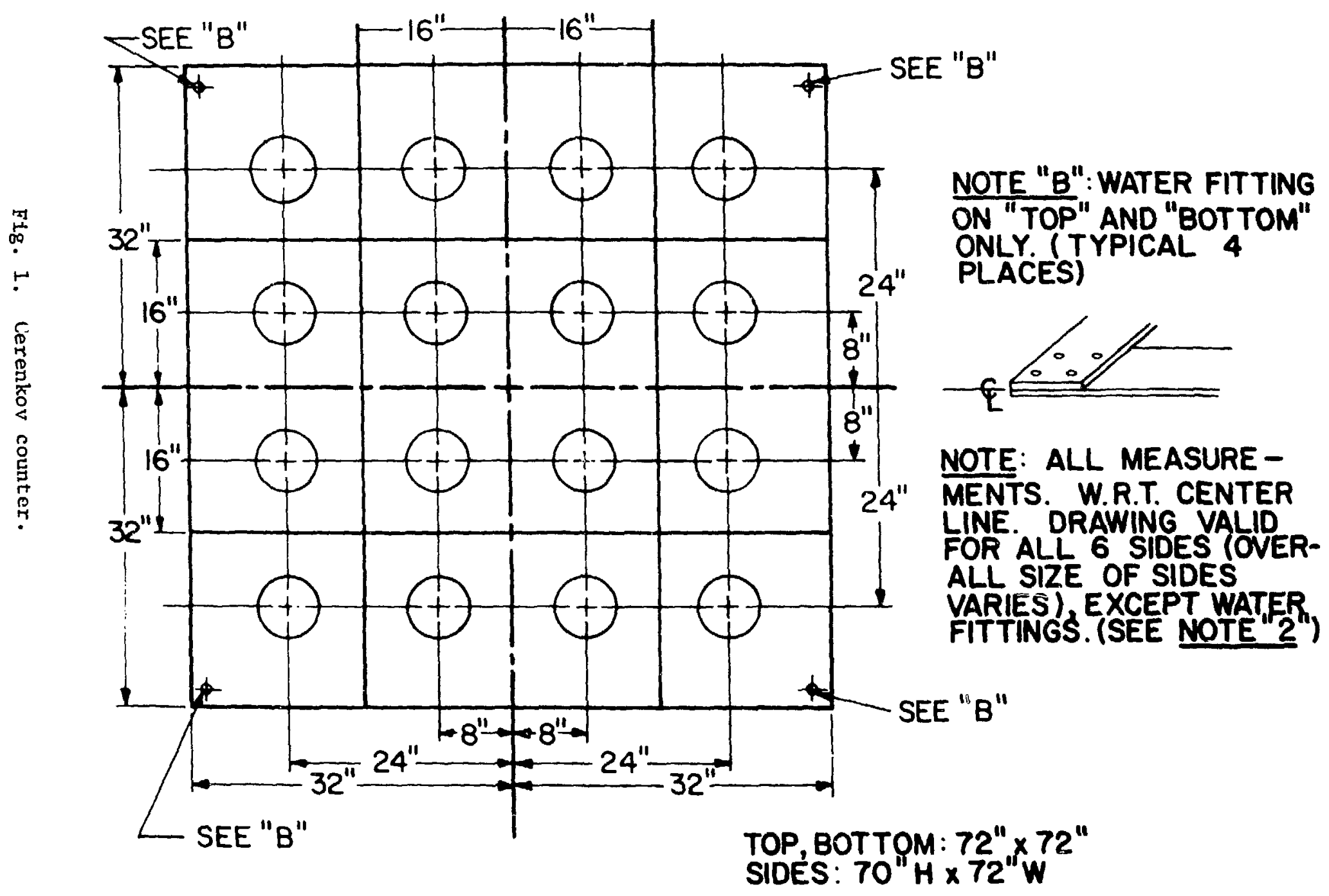




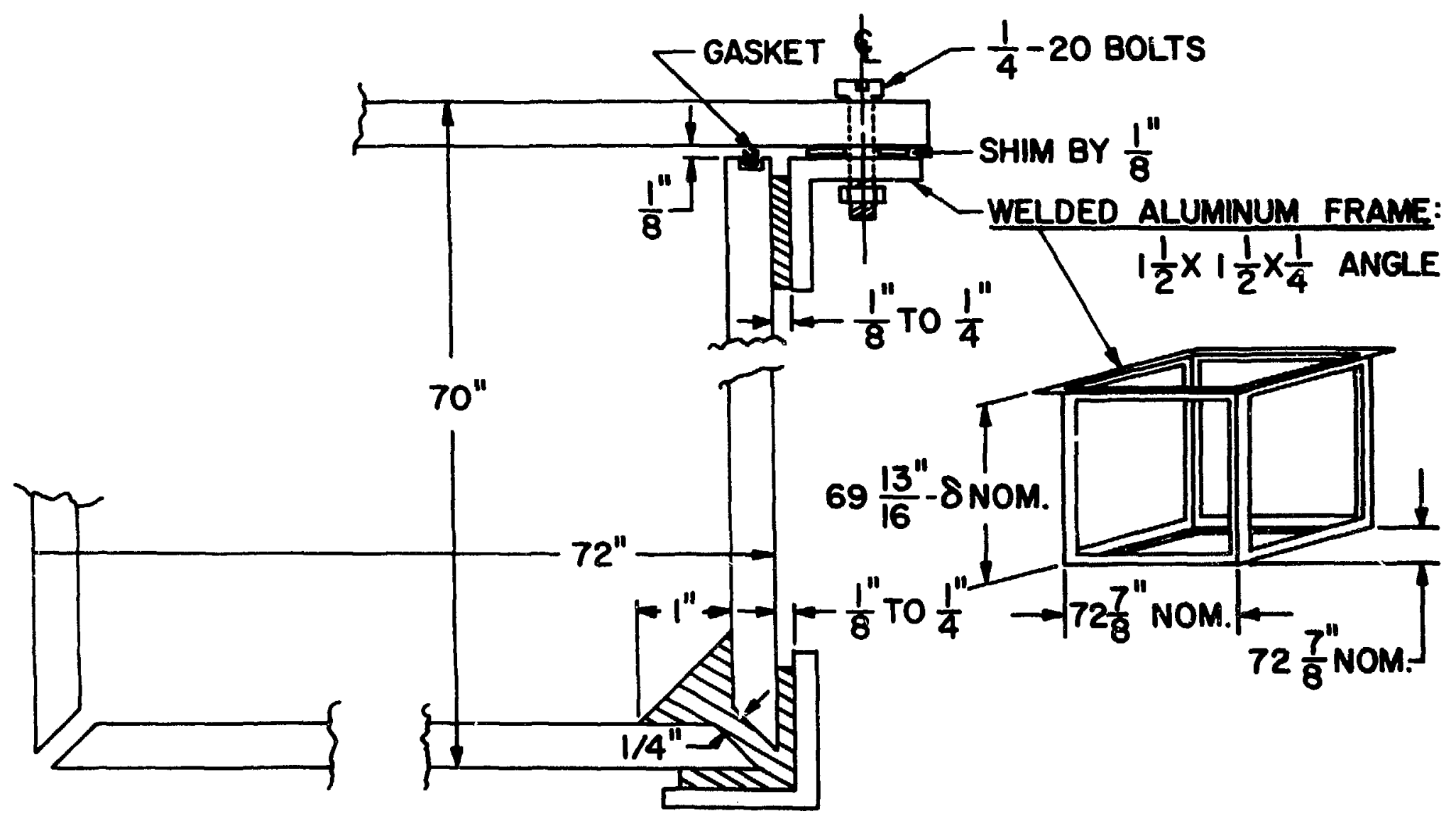

WI = CAST EPOXY EDGES 
The epoxy walls theinselves did not have sufficlent strength to withstand the hydrostatic pressure of the counter. A system of struts, 1lluecrated In Fig. 3, which were perpendicular to the walls of the counter, was used to provide mechanical integrity. The struts were cut from 1.3-cm-th1ck Benelex, 47 a cellulose lamlnate, chosen because of 1 ts exceptional stiffness (1ts modulus of elasticity $1 \mathrm{~s} 1.3 \times 10^{6} \mathrm{psi}$ ), and also because of the desire to use nonmetallic materials. The struts were slotted at their intersections, bonded to the epoxy walls, and bolted together at the corners. They held the deflection of the counter walls to legs than $3 \mathrm{~cm}$.

The portholes for the 96 phototubes were distributed in a square matrix, 16 on each of the 6 sides, in the spaces formed by the Benelex ribs. We did not use transmission windows, in order to minimize light losses; the water seal was formed directly by the $12.5-\mathrm{cm}$ tube faces. The precast epoxy porthole rings, fllustrated in Fig. 4, were cast into the epoxy walls. Each porthole ring contained an 0-ring groove and threaded inserts for attaching a matching epoxy follower ring. The follower rings are shown in Fig. 5. The phototubes were bonded into the follower rings with RTV 602 silicone rubber. ${ }^{48}$ A fiber-optics 1 ight guide, its tip bonded to the tube face, was incorporated into the assembly to allow individual testing of the tubes. The light guides went to green LEDs, three guldes per LED, which were mounted on the corners of the counter. The phototube assembly is shown in Fig. 6 .

47. Masonite Corp., $29 \mathrm{~N}$. Wacker Drive, Chicago, IL 60606.

48. General Electric Silicone Products Department, Waterford, NY 12188. 


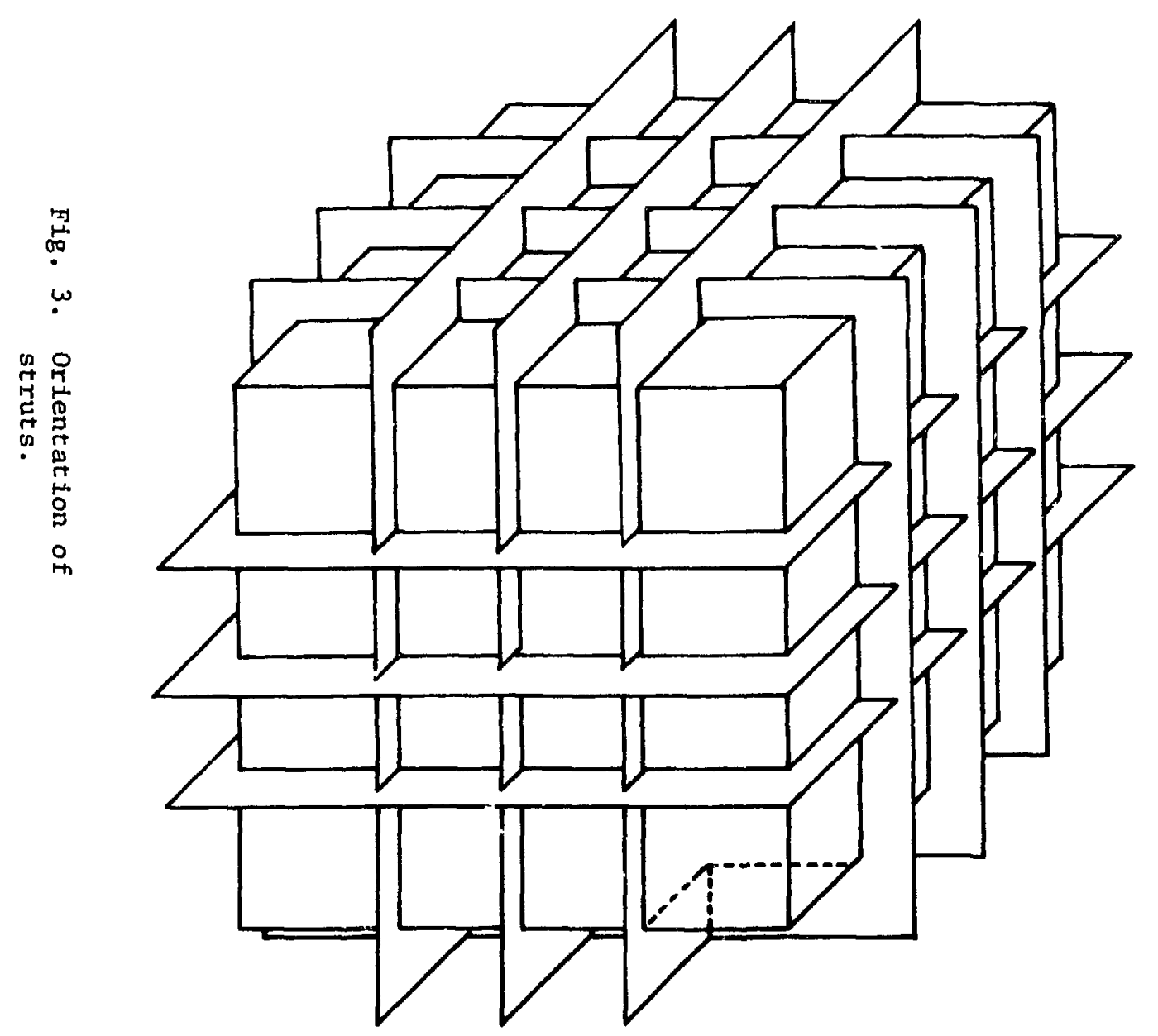

OVERALL: $90 " \times 90 " \times 88^{\prime \prime}$ HIGH ( $\left.\pm \frac{1}{4}\right)$

SIDES AND TOP: WIDE AND NARROW STRUTS.

BOTTOM: ALL WIDE STRUTS.

SIDES NOT SHOWN: SAME ORIENTATION AS FACING

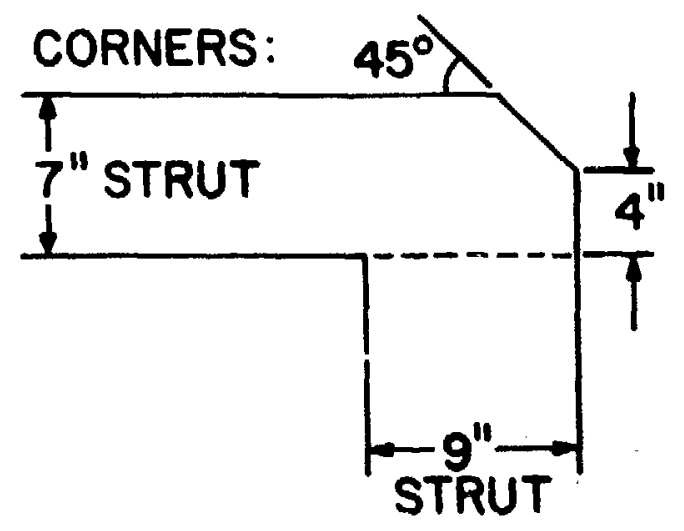



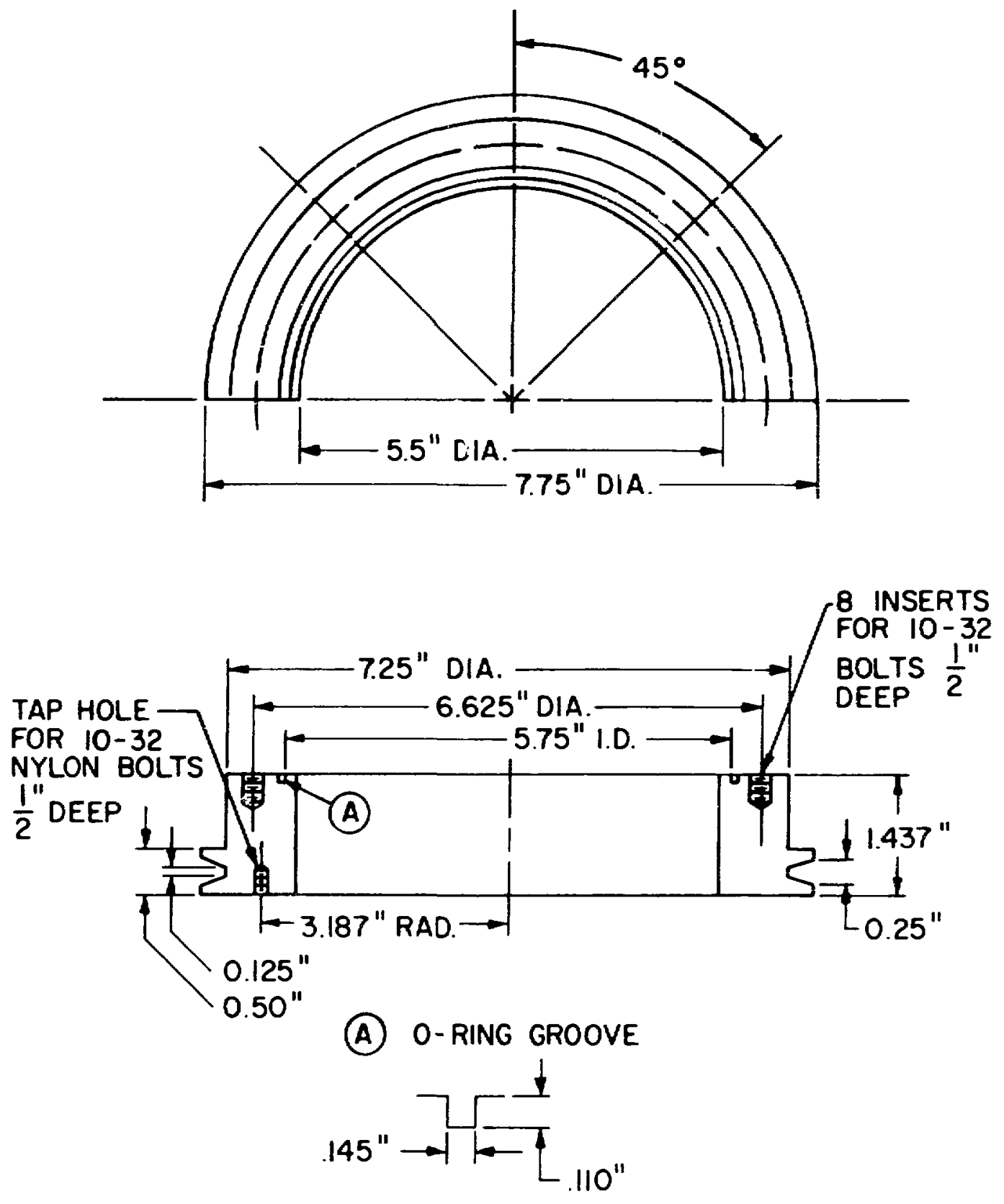

Fig. 4. Porthole ring. 

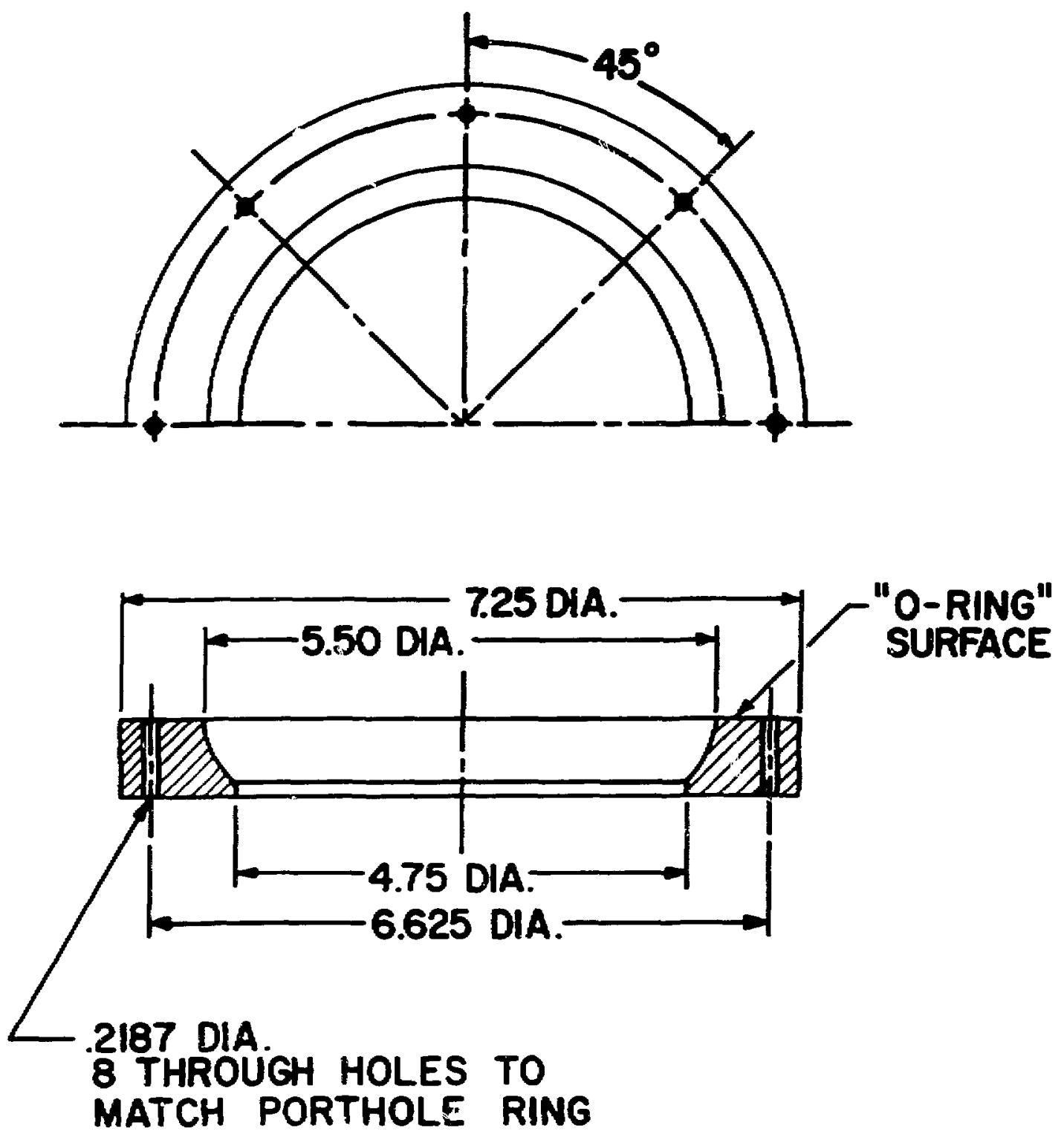

Fig. 5. Follower ring. 


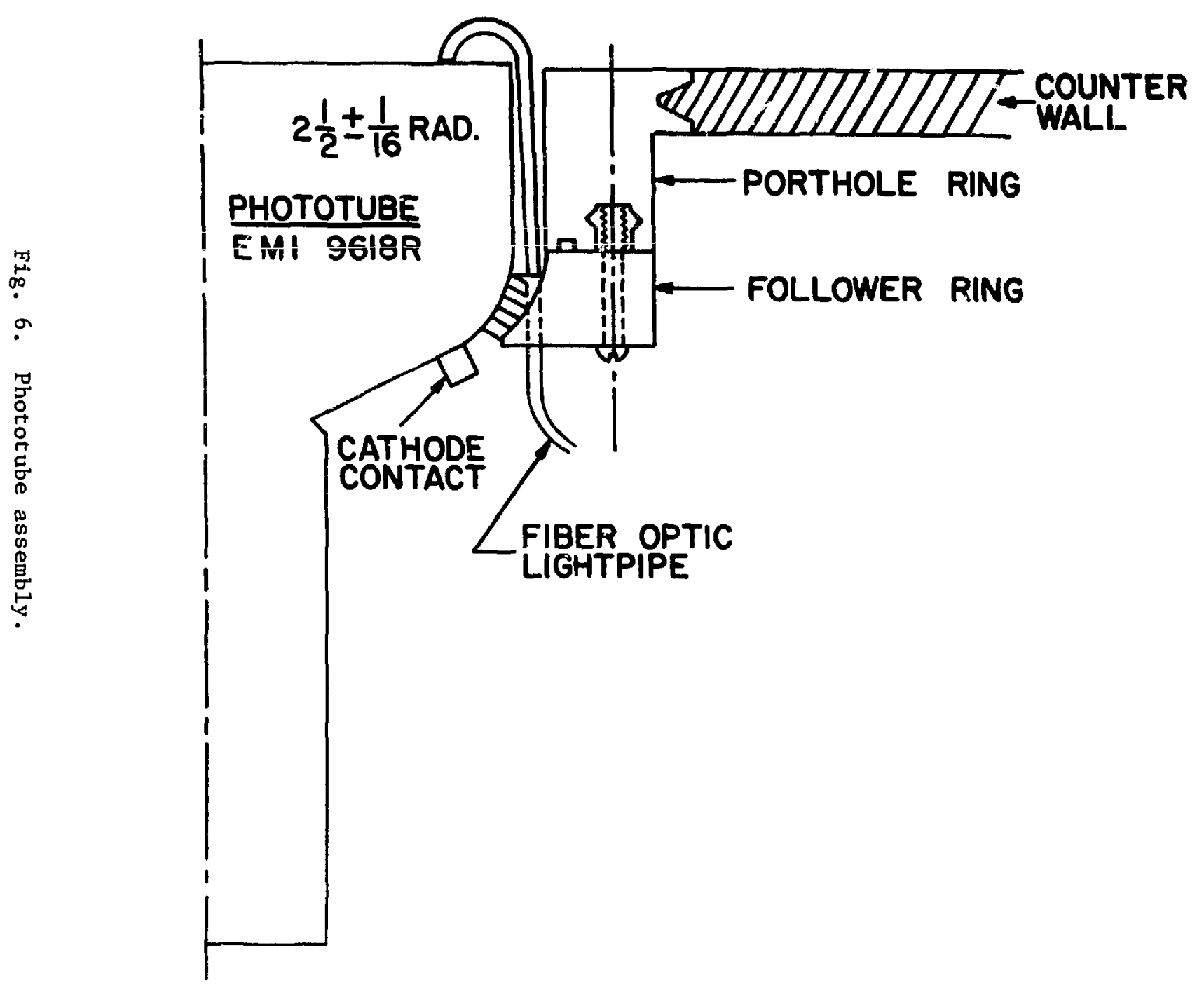


The counter was ifned with a diffuse whte reflector ande of $0.15-\mathrm{cm}^{-} \mathrm{th}$ ick Kyojex sheets, 49 with holes cut for the phototubes, and attached to the counter walls with nylon scress. Tests vere made resulting in the selection of the Rydex, using the same water and wavelength shifter solution as was used in the counter, 111uminated by an ultraviolet light, and vieved indirectly by a single phototube. The setup if Illustrated in Fig. 7. Note that the phototube was not at the angle if reflection for a smioth surface -- this was because we were Interested in measuring diffuse reflection. Table III showg the results from valious sainples, normalized to bortc actd $=100$. Boric scte and magnesium cxide are powders, and were judged to be too difficult to uae successfully underwater. The Kydex was sfmtlar to the teflon in diffuse reflectivity, and was much less expensive. The top and boctom ofdes of the cube had 2-cm-diam water fittings cast into their corners; theix location is indicated in F1g. 1. Each of the 16 porthole rings on the top had a $0.3-c r a n s 1 d e$ diameter Tygon tube 50 attached to prevent the formation of air bubbles under the phototubes. Tygon tubes also connected all the counter fittings to plastlc manlfolds with PVC valves (see Fig. 8), located outside the scintillator shield. These manifolda allowed for filling, emptying, and circulating while the counter was inaccessible behind the cosmic-ray anticoincidence shield. The opening to the air aliowed for changes in the water level due to leaks or thermal expansion or contraction, and also for alr intake and outgo while the counter was being emptied or filled.

49. Rohm and Haas Co., Johnson and Thomas St., Philadelphia, PA 19105. 50. Norton Plastics and Synthetics Division, Akron, OR 44383. 


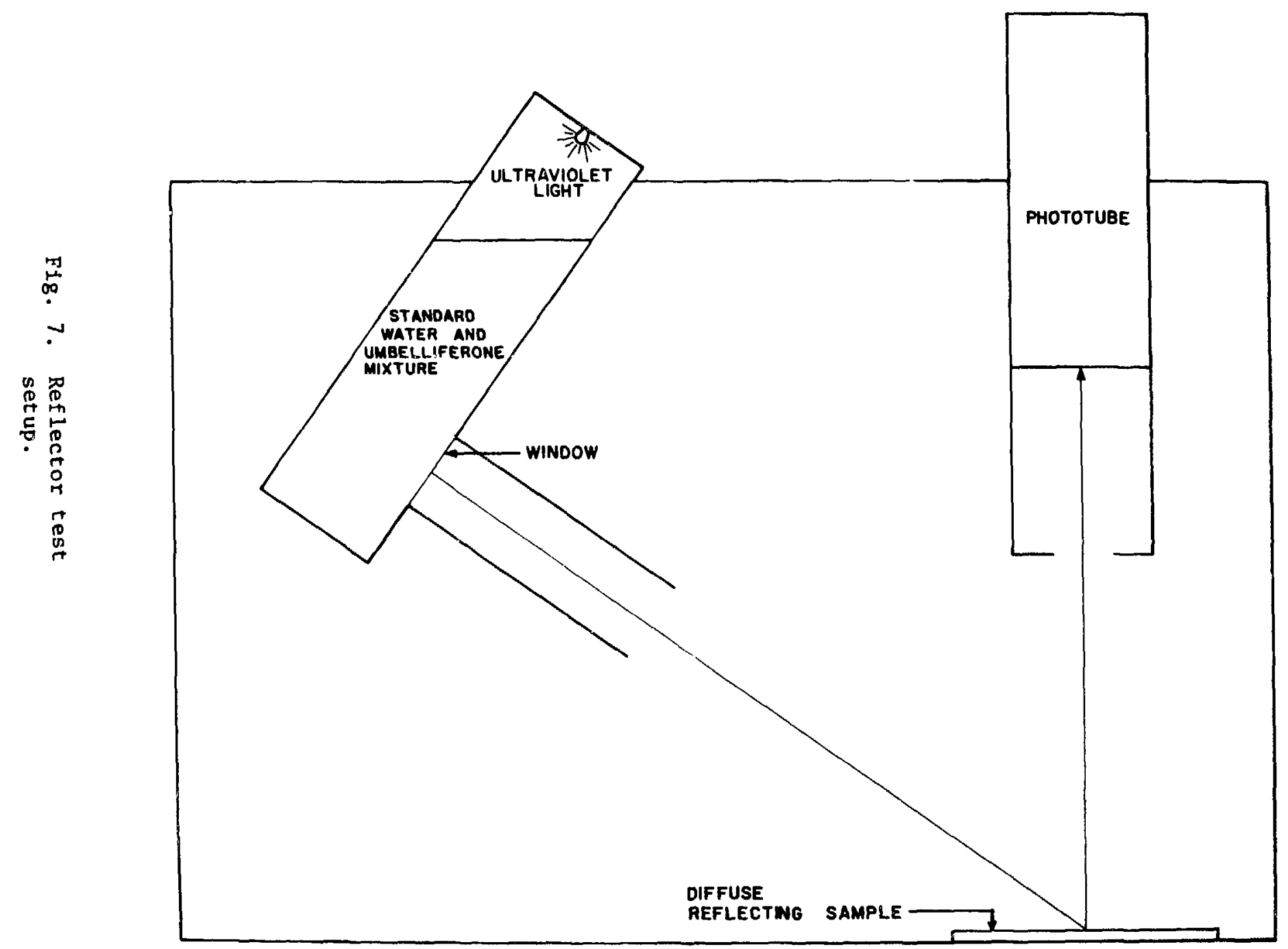

离 
TABLE III

\section{DIFFUSE REFLRCTIVITY OF VARIOUS SAMPLES}

\section{Material}

Borlc acid

Magnesium oxide

Teflon 51

Kydex

$\mathrm{ABS}^{52}$

Crumpled aluminum foll

Black sample

\section{Reflectivity}

100

92

84

82

75

77

$<3$

We always kept the upper manifold above the top of the counter, and kept a visible water level in it so we could be sure the counter was full.

In order to provide a light seal, and to trap any leaking water, the counter was wrapped in a Sears heavy-duty tarp.53 The tarp had a slot cut in it at the level of the bottom of the counter, and a plpe attached to the slot for drainage in case of a leak. The tarp was tied to the top of the counter and lald as flat as possible, so as not to interfere with the positioning of the scintillators. A sheet of black plastic covered the top and sides of the counter, where the tarp did not reach. The counter was light-tight with these coverings, even before the scintillators were moved in place.

51. E. I. du Pont de Nemours and Company, Inc., 1007 Market St., Wilmington, DE 19898.

52. Cadillac Plastic, 823 Hindsor St., Hartford, CT.

53. Catalog No. 6K75009L, Sears, Roebuck and Company, Sears Tower, Chicago, IL 60684. 

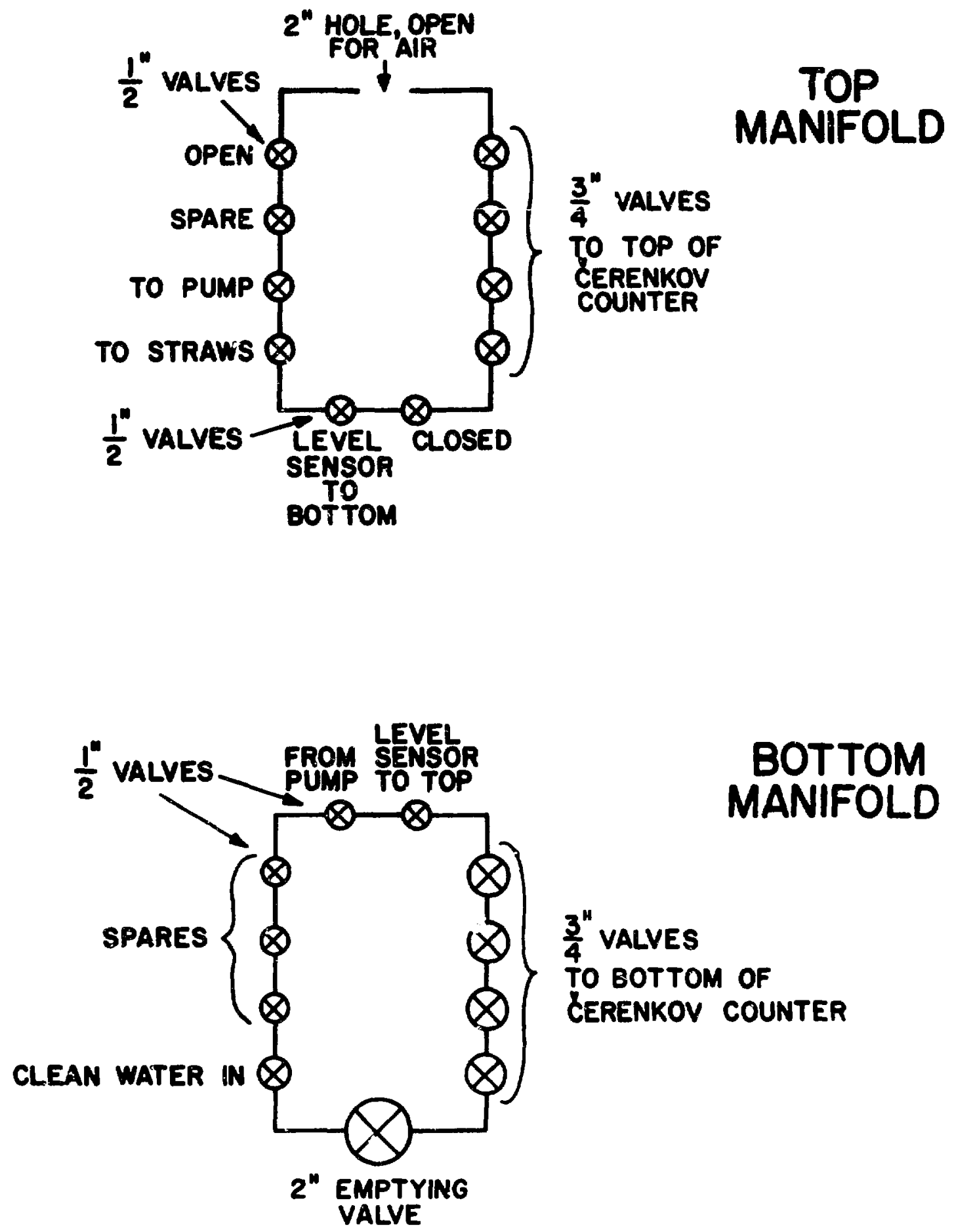

Fig. 8. Water manifolds. 
The counter was filled with water, with occasional drainings, over a perlod of four and a half years, and with heavy water for five wonths. During this period, various weaknesses in the construction showed up, in the form of cracks and breaks. The main source of problems was the Benelex rib system. Under the hydrostatic load of $200 \mathrm{~cm}$ of water $(0.2 \mathrm{~atm})$, cracks began to develop in the struts. The first cracks appeared in the narrow struts where they crossed the wide ones, gince the struts were notched In these places. Small aluminum L-bars were Installed at these crossings, for reinforcement. Then the wide struts began cracking at the crossings, and both types began cracking sit the corners. We then installed 1.6-mm-thick aluminum strips along the length of the wide struts, and also across the corners. The counter was in this condition for about one year, with no further strut problems. These splints did add about $100 \mathrm{~kg}$ of aluminum and steel to the material Inside the anticoincidence shield; the effect of this material on the observed neutrino rates is discussed in the chapter on backgrounds.

Partly as a result of strut fallure and partly from casting stresses around the porthole rings, cracks appeared in the epoxy walls themselves. These cracks were repalred from the outside of the counter, using fiberglass tape and epoxy patches. Fortunately, none of these cracks was sertous enough to cause a major water leak. Leaks around the phototubes developed as the counter was filled and emptied; these were generally around the screws or 0 -rings, and were repatred as they developed. 
Due to the relatively long path lengths involved in light transmission through the counter, we required a high degree of transparency of the water. Tap water was run through two lon-exchange resing 54 1r. series, and then through a particulate filter. The principle behind the operation of the ion-exchange resins is that they replace heavy positive lons in the water by $\mathrm{H}^{+}$, and heavy negative Ions by $\mathrm{OH}^{-}$; these then combine to $\mathrm{H}_{2} \mathrm{O}$. Most impurities in water exist in Ionic form. The resistivity was found to be more than $10^{7} \Omega \mathrm{cm}$. Measurements of throughgoing cosmic-ray muons Indicated that transmission of light from one side of the counter to the other, sn average distance of $150 \mathrm{~cm}$, was $55 \%$. Th1s gave an absorption length $(1 / \mathrm{e})$ of $250 \mathrm{~cm}$.

In order to maximize the number of photoelectrong emitted by the phototubes, we used a wavelength shifter in the vater. We chose 4-methyl-umbelliferone, with an absorption peak at $360 \mathrm{~nm}$ and an emission peak at $450 \mathrm{~nm} .55 \mathrm{Th} 1 \mathrm{~s}$ chemical will not fluouresce unless it is In a solution whose $\mathrm{pH}$ is at least 8.0 . To achleve this, we ured $87.5 \mathrm{mg} / \ell$ of ammontum phosphate $\left.\left[\left(\mathrm{NH}_{4}\right)_{2} \mathrm{HPO}_{4}\right)\right]$ and $16.7 \mu \ell / \ell$ of 587 ammonia solution $\left(\mathrm{NH}_{3}+\mathrm{H}_{2} \mathrm{O}\right)$. This amount of additive had no effect on the transparency of the water. Figure 9 is a curve showing the gain of the counter, In arbitrary units, vs the umbelliferone concentration. We used $0.5 \mathrm{mg} / \mathrm{l}$ as our standard concentration.

54. Cul11gan USA, 1 Cu111 gan Parkway, Northbrook, IL 60062.

55. D. H. Leaback and P. G. Walker, Blochemical Jour. 78, 151 (1961);

N. A. Porter, Nuovo CImento 5, 526 (1957). 


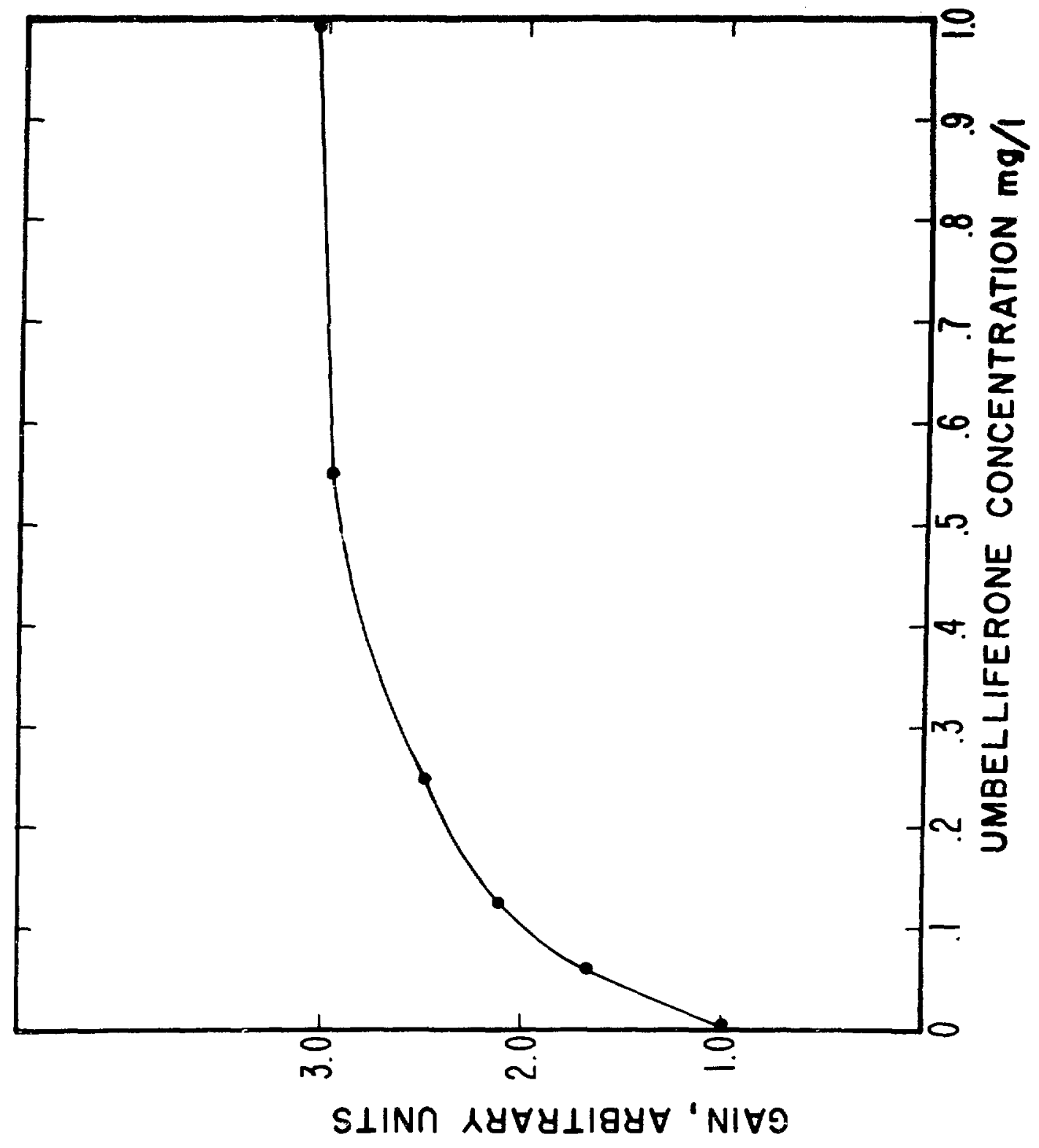

Fig. 9. Umbelliferone concentration curve. 
During the months of June through September 1978, we ran the counter filled with $\mathrm{D}_{2} \mathrm{O}$, rather than $\mathrm{H}_{2} \mathrm{O}$. The heavy water was borrowed from Savannah River, 56 and we wanted to winimize the amount lost in transportation, storage, and use, so as to minimize the cost of replacing 1t. Because of the Cerenkov counter's long history of repairs, we decided we needed a passive recovery system in the case of any leak, and also an alarm system 80 positive action could be taken to reduce water 1088 in the event of a spill. A tank large enough to hold all the heavy water wes installed in the hill south of the neutrino house, and a pipe installed connecting it to the outlet of the tarp enclosing the counter. Two water-sensitive detectors were placed in the plpe and connected to two alarms; in the presence of water, the detectors would close a clrcult and set off the alarms. A level sensor was placed in the overflow bottle and set to close a circuit if the water level dropped more than $5 \mathrm{~cm}$ below 1 ts nomfnal level; this c1rcuft was hooked up to one of the two alarms. The alarm with both the water-sensitive detector and the level sensor was placed in the electronics traller; it sounded an audible alarm and also brosdcast a tone on the frequency of a pocket pager, which we carrled when we were not in the traller. The second alarm was in the accelerator control room; it set off an audible alarm, and the accelerator operators had Instructions to page a second pocket pager, which we also carried around, and also to announce over the public address system if the alarm had gone off. The 5-cm emptying hose from the bottom manifold was also connected to the outside tank, so we had the option of emptying the

56. E. I. du Pont de Nemours and Co., Savannah RIver Plant, Alken, SC 29801 . 
counter if a leak occurred. Fortunately, no leaks did occur, and the alarm system never gave a false alarm. We purified the heavy yater through a set of lon-exchange resins, 57 a carbon filter, and a particulate filter, as we were filling the counter. We used the same concentrations of umbellfferone, ammonium phosphate, and ammonia as we used in light water, and filtered the heavy water again on emptying to remove the chemicals we had added.

The phototubes we used on the Cerenkov conter were $12.5-\mathrm{cm}$ EMI 9618R tubes.58 The base circult is shown in Fig. 10. The focus was adjusted Individually for each tube; for the best focussing the rise time of the anode signal was $30 \mathrm{~ns}$ and the fall time $70 \mathrm{~ns}$. The gain for high voltages around $1500 \mathrm{~V}$ was such that one photoelectron corresponded to a 4-mV pulse helght into $50 \Omega$. We measured both the gain of each phototube and the relative quantum efficiency of 1ts photocathode. To do this, we used two different 11ght sources: an alpha source, ${ }^{241} \mathrm{Am}$, mounted on a plastic scintillator, and a light-emitting diode. Both Ifght sources were mounted in a mechanfcal device ensuring a well-defined position with respect to the phototube under measurement. The high voltage of each tube was adjusted to yleld a given pulse height for the alpha source. The LED was then adjusted to give the same pulse helght. Assuming that the width of the LED peak was essentially due to photostatistics, one could then find the average number of photoelectrons at the alpha peak:

57. Catalog No. COMB-012-04, Mil11pore Corp., Bedford, MA 07130.

58. EMI Gencom, 80 Express St。, Plalnview, NY 11803. 


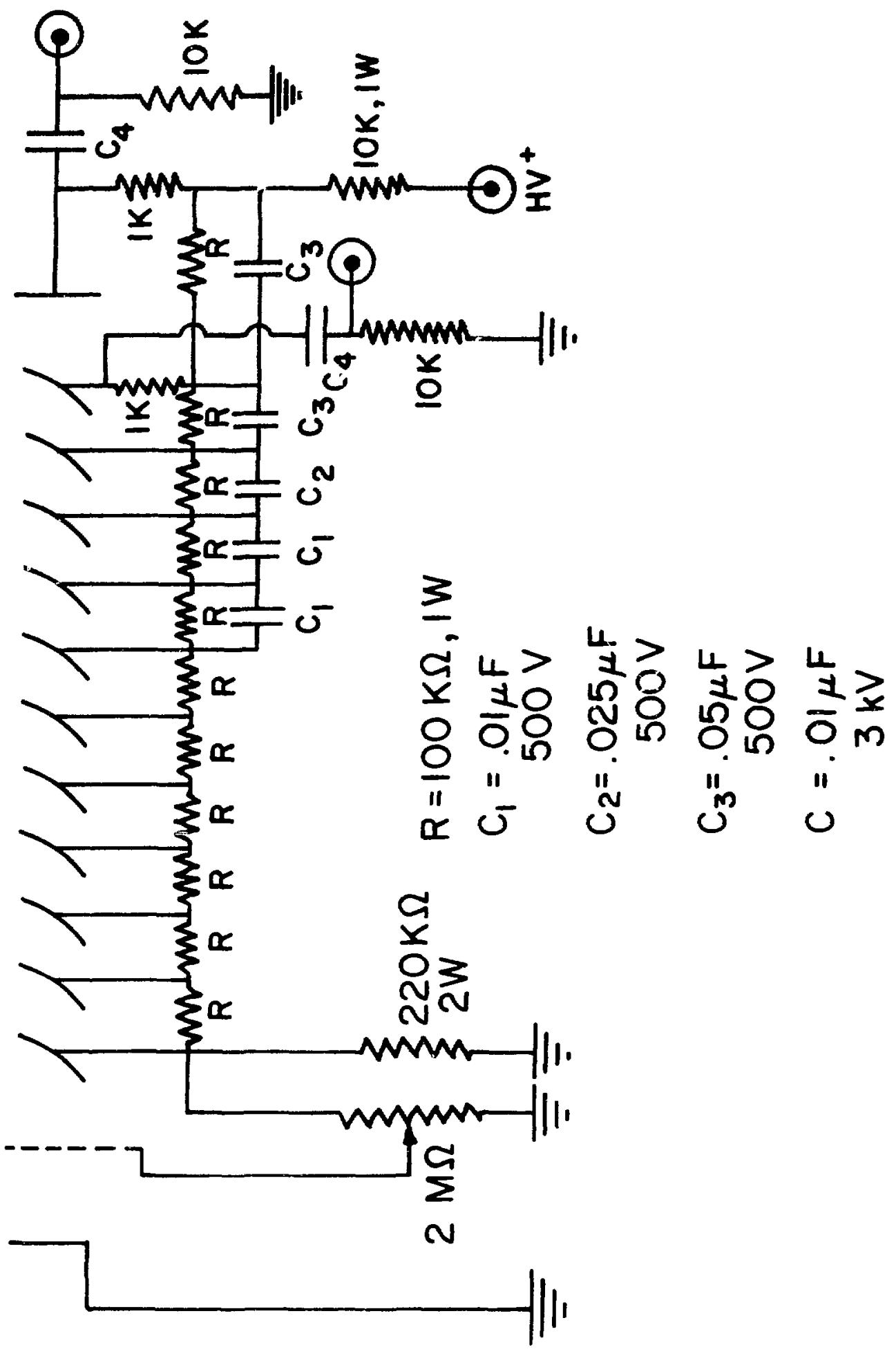

Fig. 10. Circuit diagram, EMI 9618R phototube base. 


$$
\mathrm{N}_{\mathrm{pe}}=1, \mathrm{\sigma}^{2}
$$

where $\sigma=$ FWHM/2.3. Once th1s number 18 known, it was possible to calibrate the gain of the Cerenkov counter in photoelectrona/MeV. Table IV shows this gain for the 16 top tubes, the 16 bottom tubes, and the 96 tubes, as a function of reflector and wavelength shifter. The top/bottom ratio measured the isotropy of the light (these measurements were done with throughgolng vertical cosmic rays) and was increased, as expected, by adding the reflector and the wavelength shifter. The total number of 5.3 photoelectrons per MeV for the final setup gave a satisfactory resolution. Around $30 \mathrm{MeV}$ the photostatistics were comparable to the edge effects and radiation losses. The Monte Car1o spectrum in Fig. 37 (see p.118) Includes a resolution of $12 \%$, as well as edge effects and radiation 1osses, which was found to be the begt fit to the measured muon decay spectrum.

TABLE IV

GAIN OF THE CERENKOV COUNTER AS A FUNCTION OF REFLECTOR AND WAVELENGTH SHIFTER

Conditions

Reflector Shifter

No

No

No

Yes
Photoelectrons/MeV

Top Bottom Total

$\begin{array}{lll}<0.1 & 0.6 & 1.1\end{array}$

$\begin{array}{lll}0.3 & 0.7 & 1.8\end{array}$

$\begin{array}{lll}1.1 & 1.5 & 5.3\end{array}$ 


\section{B. SCINTILIATORS}

The Cerenkov counter was surrounded on all six sides by a cosmlc-ray anticolncidence shield, consisting of both active and pasaive elements. The innermost layer consisted of scintillators, overlapped so that particles could not get between then. The scintiliators thenselves were sheets of Pilot $Y$, manufactured by Nuclear Enterprises.59 Each sheet was $2.5-\mathrm{cm}$ thick, 60-cm wide, and $260-\mathrm{cm}$ long. There vere trapezoldal light pipes on each end, also 2.5-cm thick. Half of the scintillators had their light pipes mounted at a $90^{\circ}$ angle to the scintillator; the other half were flat. A 12.5-cm-square, 1.3-cm-thick plastic plate was glued to the end of each lightpipe, and a 5-1n.-diam phototube mounted on each plate. Figure 11 shows the scintillator sheets, 11ghtpipes, and $90^{\circ}$ bend prisms. Half of the bent scintillators had the longer $90^{\circ}$ bend plece indicated.

The side scintillators were mounted on steel support stands which held them in position and also held the lead which was mounted outside them. The bottom scintillators sat on the table directly under the Cerenkov counter, and the top scintillators sat on the support struts on top of the counter. Figures 12 and 1.3 show the horizontal (bent; east and west sides) and vertical (flat; north and south sides) scintillators, their stands, and a partial view of the lead mounted behind them. Figure 14 is a top view showing how the stands fit together. The top lead did not rest on the top scintillators, but was placed in a framework which was supported at its corners by the side stands. There was a second layer of scintillators on the top, which was Identical to the first layer, and which rested directly on the top lead. 


\section{SCINTILLATOR}

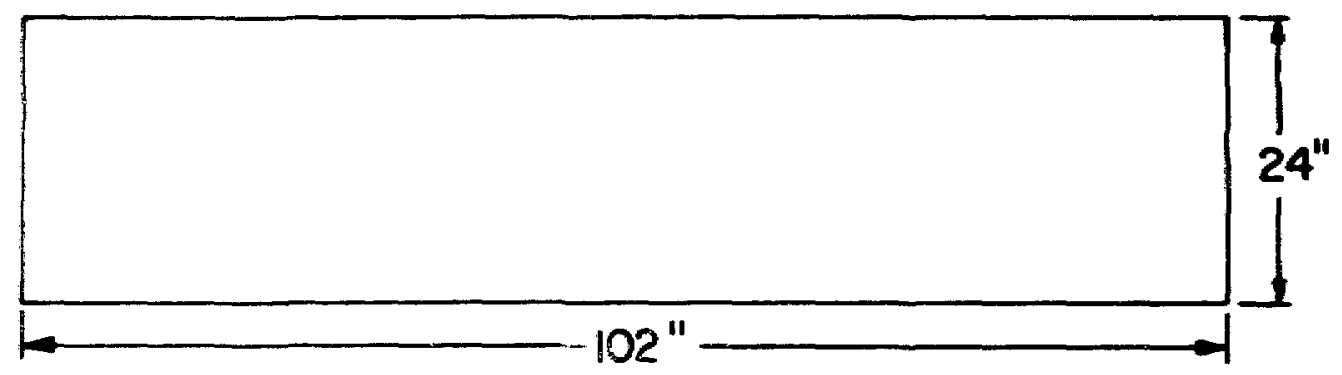

LIGHT PIPES
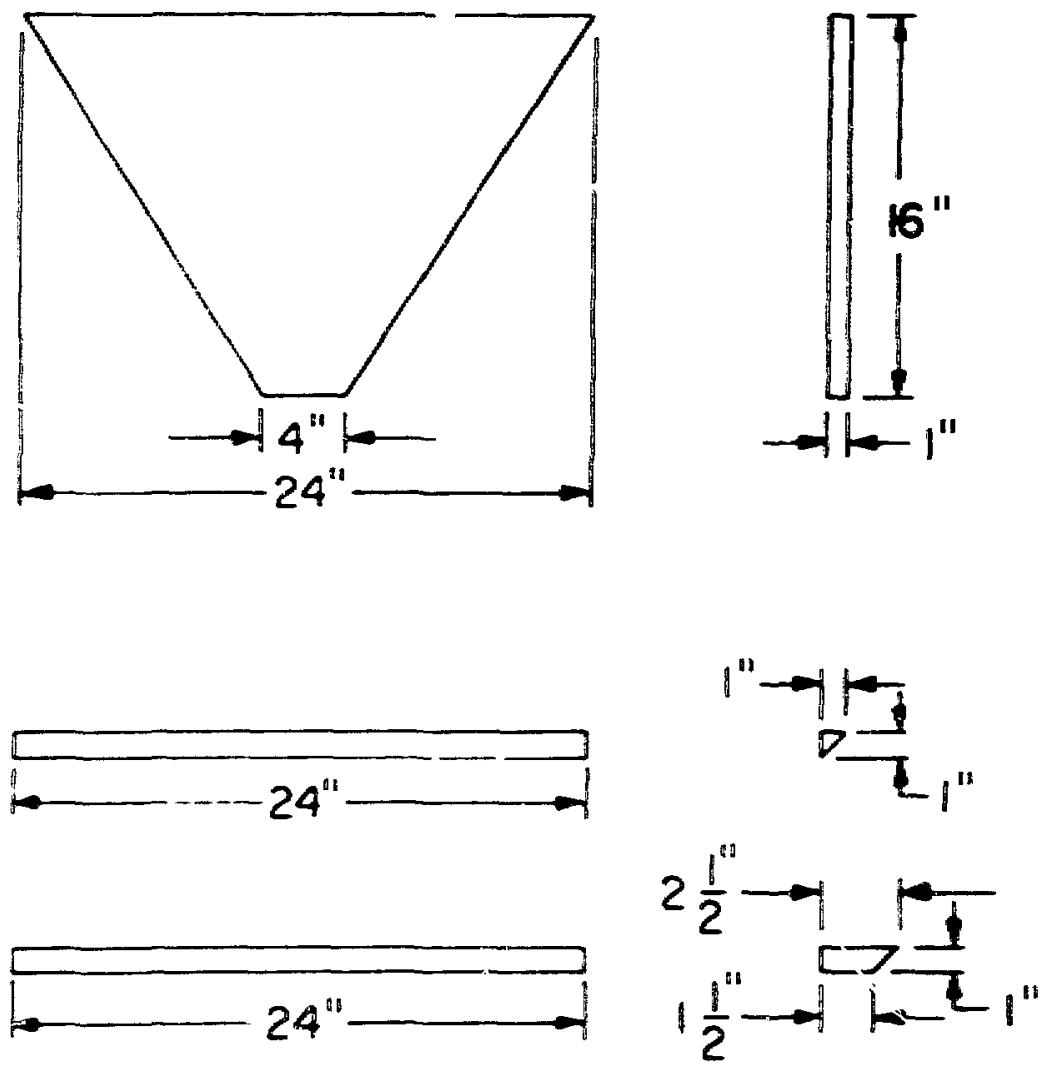

Fig. 11. Scintillatoz, Iight

pise, and $90^{\circ}$ bend 


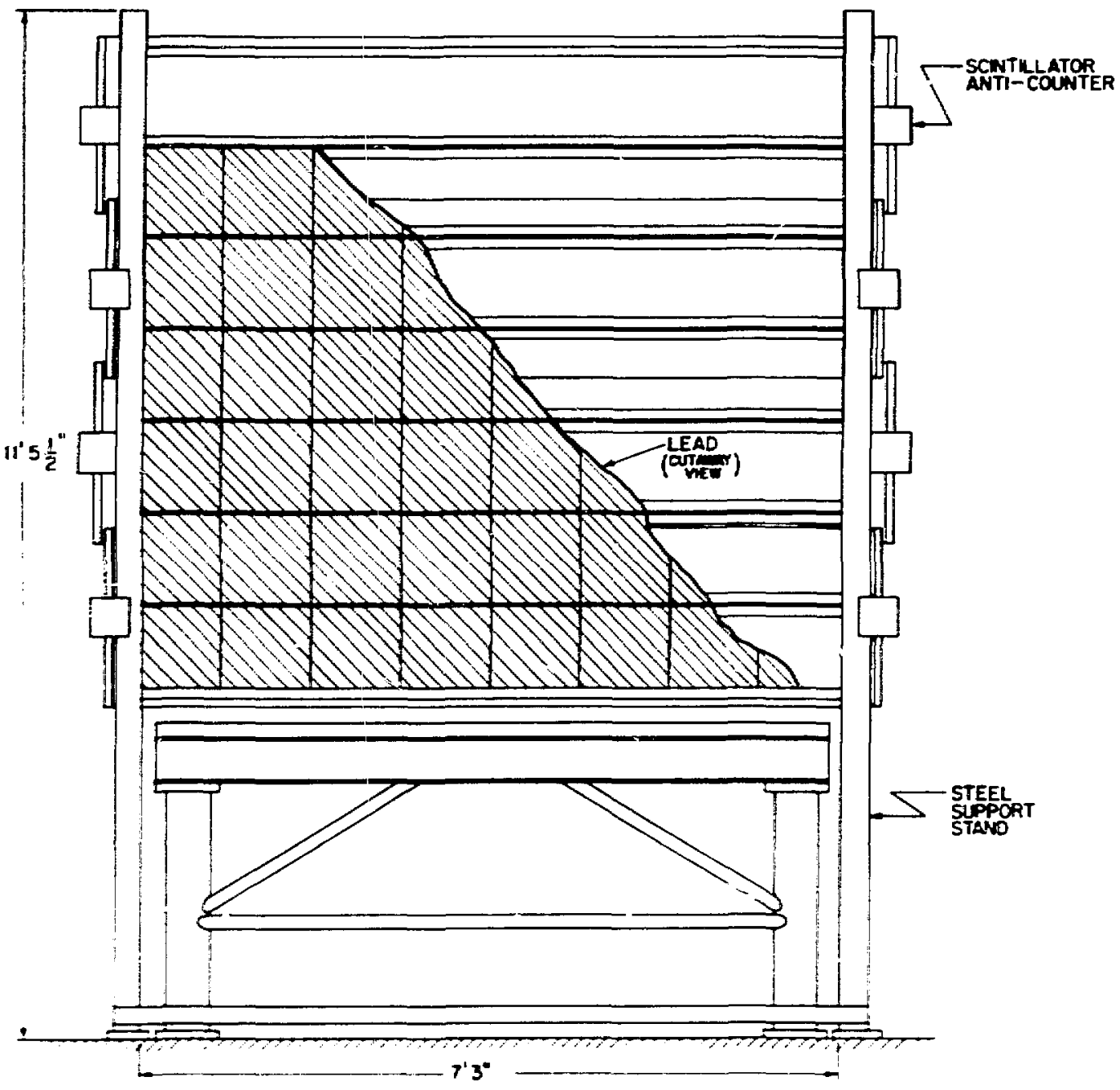

$=$

Fig. 12. Side scintiliators, horizontal. 


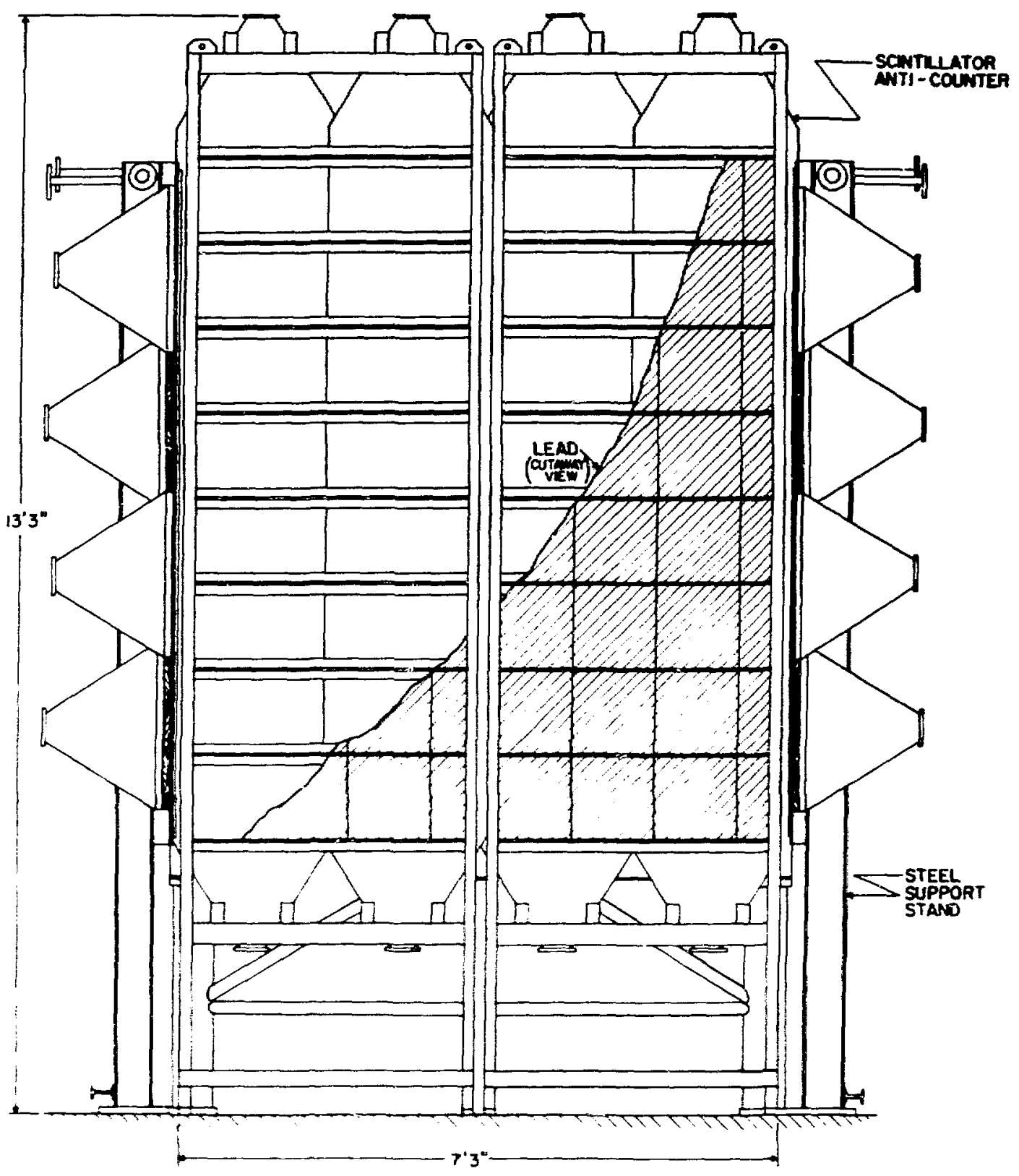

Fig. 13. Side scintillators, vertical. 


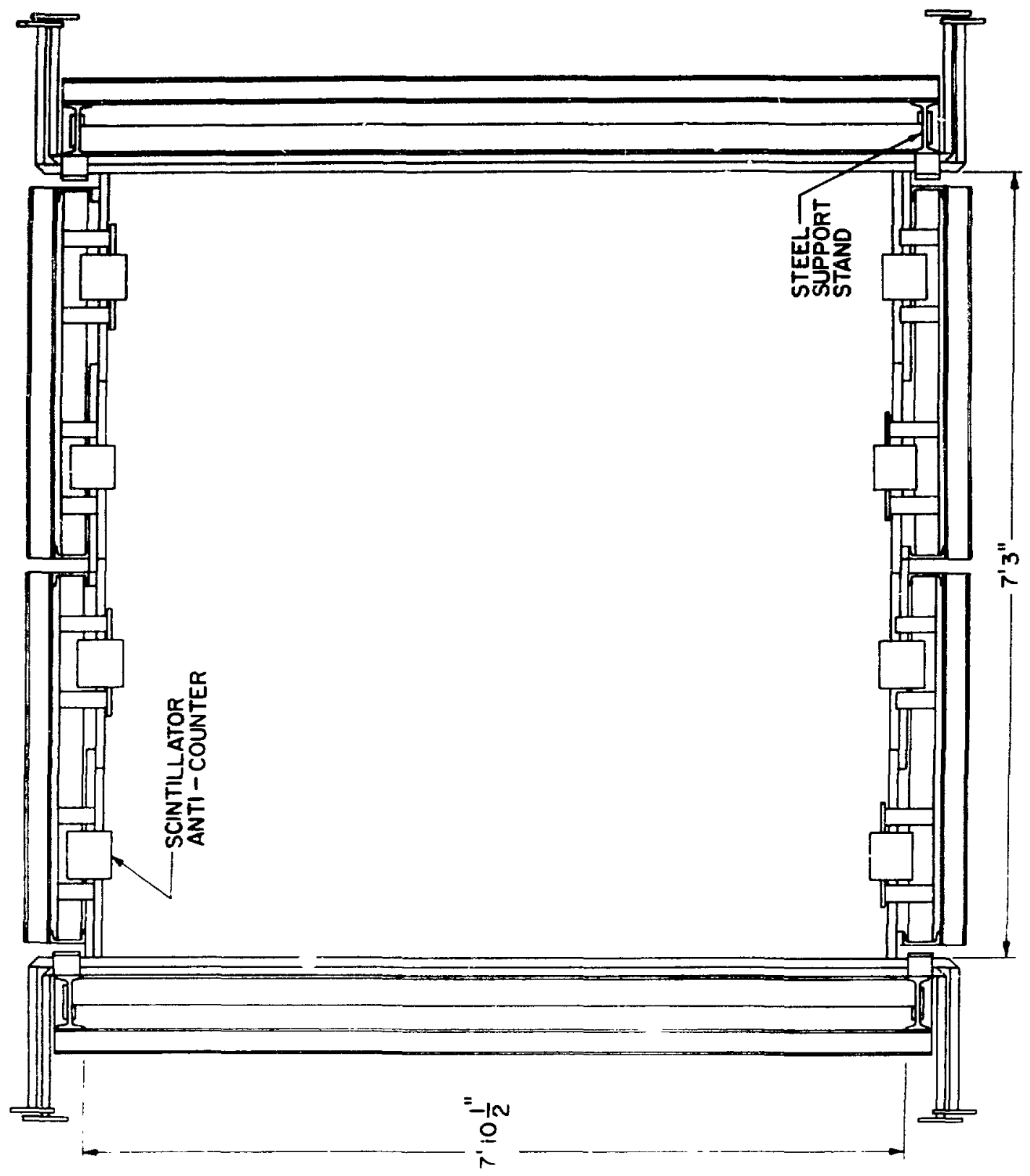

Fig. 14. Top view, scintillator stands. 
Figure 15 shows the top scintillators and their position relative to the side scintillators. Table V lists each side of scintillators with its designation.

The phototubes used on the scintillators were RCA 4525, 12.5-ca tubes. 60 These tubes had only ten stages, and the signals needed amplification; an amplifier was therefore bullt Into each base. 61 Figure 16 is a diagram of the base. The rest of the electronics for the scintillators is described in the electronics section. Each tube had an LED mounted on tha lightplpe near 1t, which allowed for testing of all the tubes.

\section{TABLE V}

NOMENCLATURE OF SCINTILLATORS

$A_{1}$ Inner layer of top scintillators

$\mathrm{A}_{2}$ North side

$\hat{A}_{3}$ West side

A4 South side

A 5 East side

A6 Bottom

A Second layer of top scintillators

60. Radio Corp. of America, Electro-Optics and Devices, New Holland Ave., Lancaster, PA 17604

61. Amplifier design by J. Studebaker, Los Alamos Scientific Laboratory report LA-5749-MS (1974). 


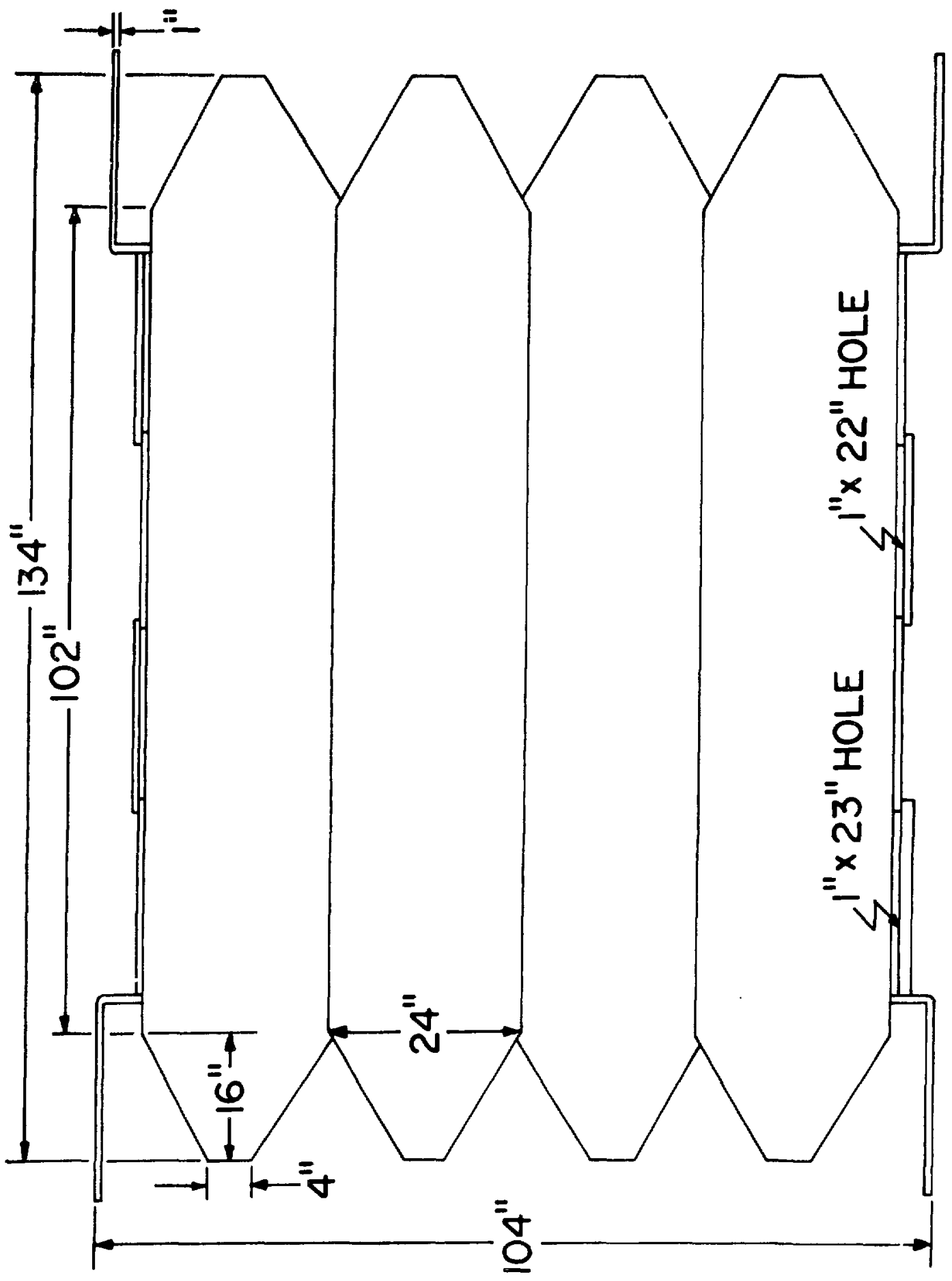

Fig. 15. Top scintillators. 


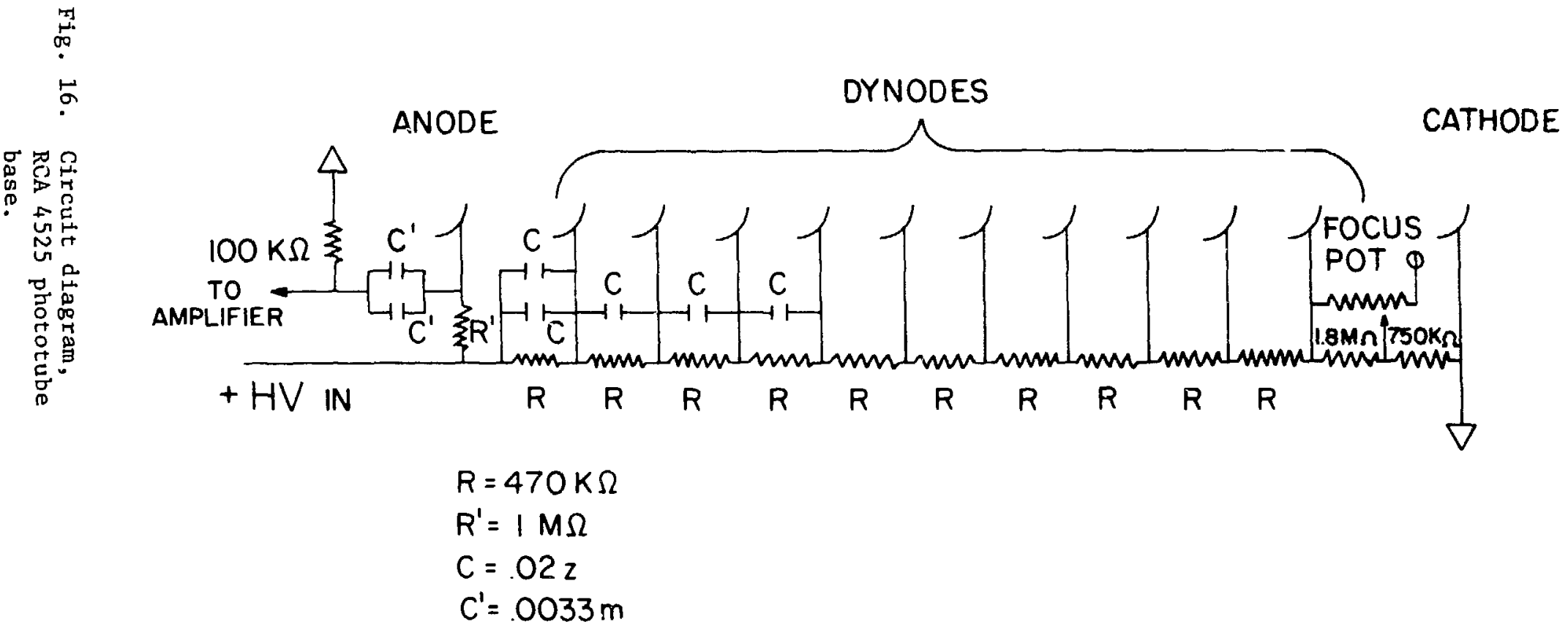




\section{DRIFT CHAMBERS}

The drift chambers were approximately 240-cm square, with two of the chambers alightly larger in one dimension. The larger ones were on the south and north sides, and the smaller ones on the east and weist. The wire spacing in all chambers was $10 \mathrm{~cm}$; the larger chambers had 25 wires, the smaller 24. The wires were 3.9-mil molybdenum with 67 gold bond. There were also fleld shaping wires between the sense wires. We ran with $+4800 \mathrm{~V}$ on the sense wires and $-1000 \mathrm{~V}$ on the field wires. The gas mixture was $80 \%$ methane and $20 \%$ argon, and wis kept an output pressure of $6 \mathrm{~mm}$ of ofl. Drift times were on the order of $1 \mu \mathrm{s}$; we were not sensitive to their exact magnitude.

The chambers were mounted on the north, east, and west sides directly outslde the lead; the south chamber was at an angle, as described in the chapter on backgrounds. They were supported on brackets bolted to the scintillator stands. Figure 17 is an exploded dlagram of the entire experimental setup with everything in place.

We measured the efficlency of the drift chambers using some small scintillators as a trigger, and found it to be at least 99\%. The singles rates in each chamber, measured in the neutrino house with the beam off, were around $800 / \mathrm{s}$. Unfortunately, we frequently had trouble with radioactive gas from the beam stop getting into the neutrino house. This affected the drift chambers most serlously, with rates as $h f_{b} \hat{i}$ as $50 \mathrm{k} / \mathrm{s}$ in a single chamber. Since we created an $11-\mu \mathrm{s}$ anticoincidence pulse from the drift chamber output, these high rates led to enormous dead-time problems. We attempted to seal the neutrino house to keep the gas out, as described in the next section; we also made live-time cuts on the data, as described In the section on data analysis. 
SCINTILLATOR

ANTI-COUNTERS

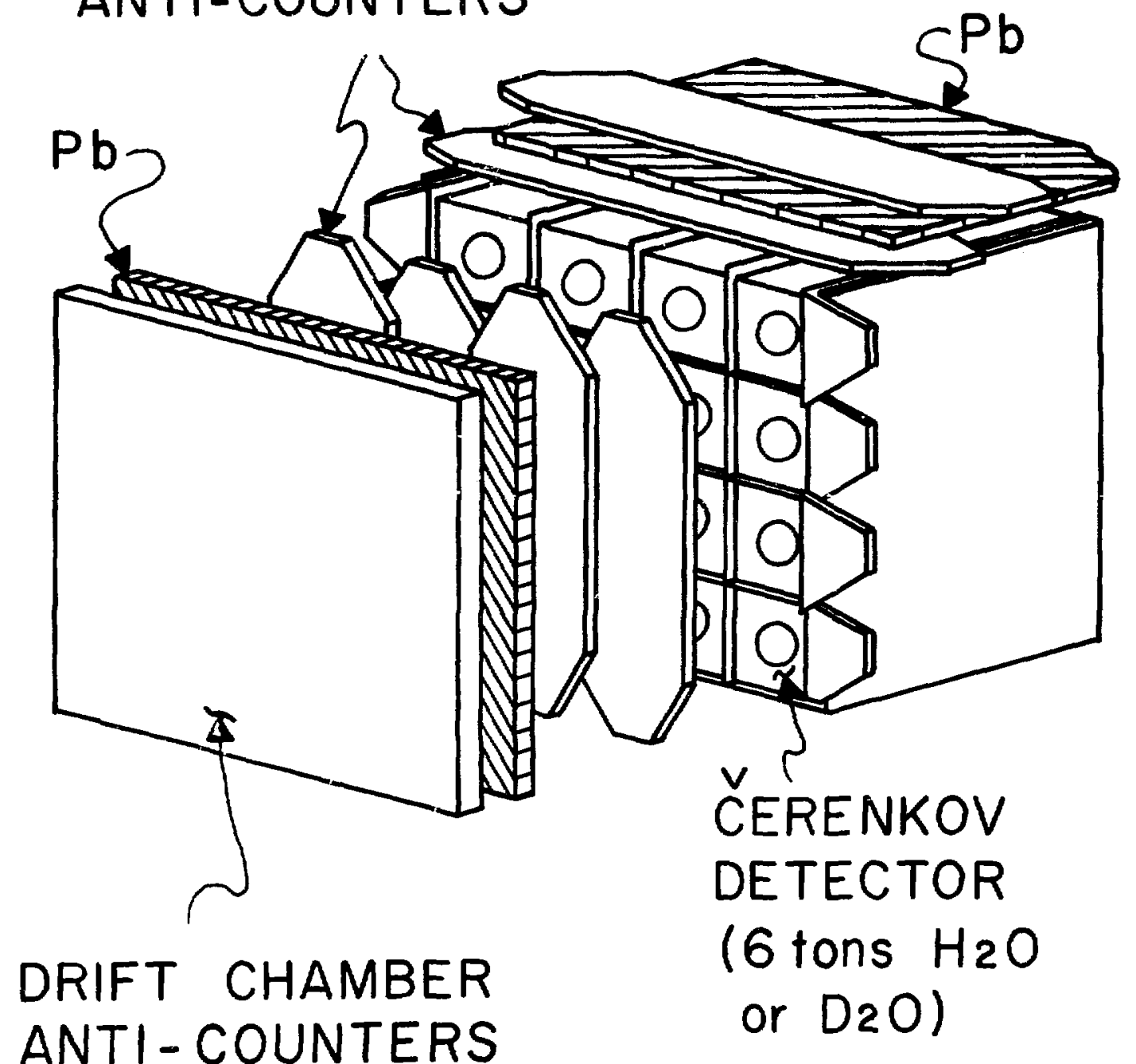

Fig. 17. Exploded view of experimental setup. 
CHAPTER 3

THE LAMPF ACCELERATOR AND THE NEUTRINO HOUSE

\section{A. THE LAMPF ACCELERATOR}

Figure 18 shows the LAMPF experimental areas, including the neutrino area. LAMPF $1 \mathrm{~s}$ an $800 \mathrm{MeV}$ linear proton accelerator, with a design intensity of $1 \mathrm{~mA}$ of $\mathrm{H}^{+} ; \mathrm{H}^{-}$lons are also accelerated simultaneously at a much lower intensity. The macroscopic duty factor Is 6\%; this consists of a 500-us macropulse at 120 cycles per second. Higher duty cycles are also possible, and are achieved by lengthening the macropulses. The highest duty cycle we ran at was $7.5 \%$, although plans are to go as high as 12\%. The switchyard at the end of the accelerator provides beam to the four main beam Iines: Line A gets most of the $\mathrm{H}^{+}$beam; Lines $\mathrm{B}$ and $\mathrm{C}$ use $\mathrm{H}^{-}$; Line $\mathrm{D}$ uses part of the $\mathrm{H}^{+}$beam, efthe: an entire macropulse ( 1 in 10,1 in 12 , and 1 in 120 are common) or the first portion ("front porch") of each macropulse. There are four production targets in Line A: a thin target used for Irradiation and nuclear-chemistry studies; Target A-1, 3-cm carbon, which provides pion beams for the Low-Energy Pion channel. (LEP) and the Energetic Pion Channel and Spectrometer (EPICS); Target A-2, 5-cm carbon, which provides a high-energy pion beam for the pion and particle physics channel $\left(\mathrm{P}^{3}\right)$ and a muon beam for the Stopped Muon Channel (SMC); and Target A-5, 8-cm carbon, whlch provides a pion beam used for blomedical research. Targets $A-1$ and $A-2$ are in the beam continuously (unless there 1s some problem); the blomedical channel, however, has no beam 


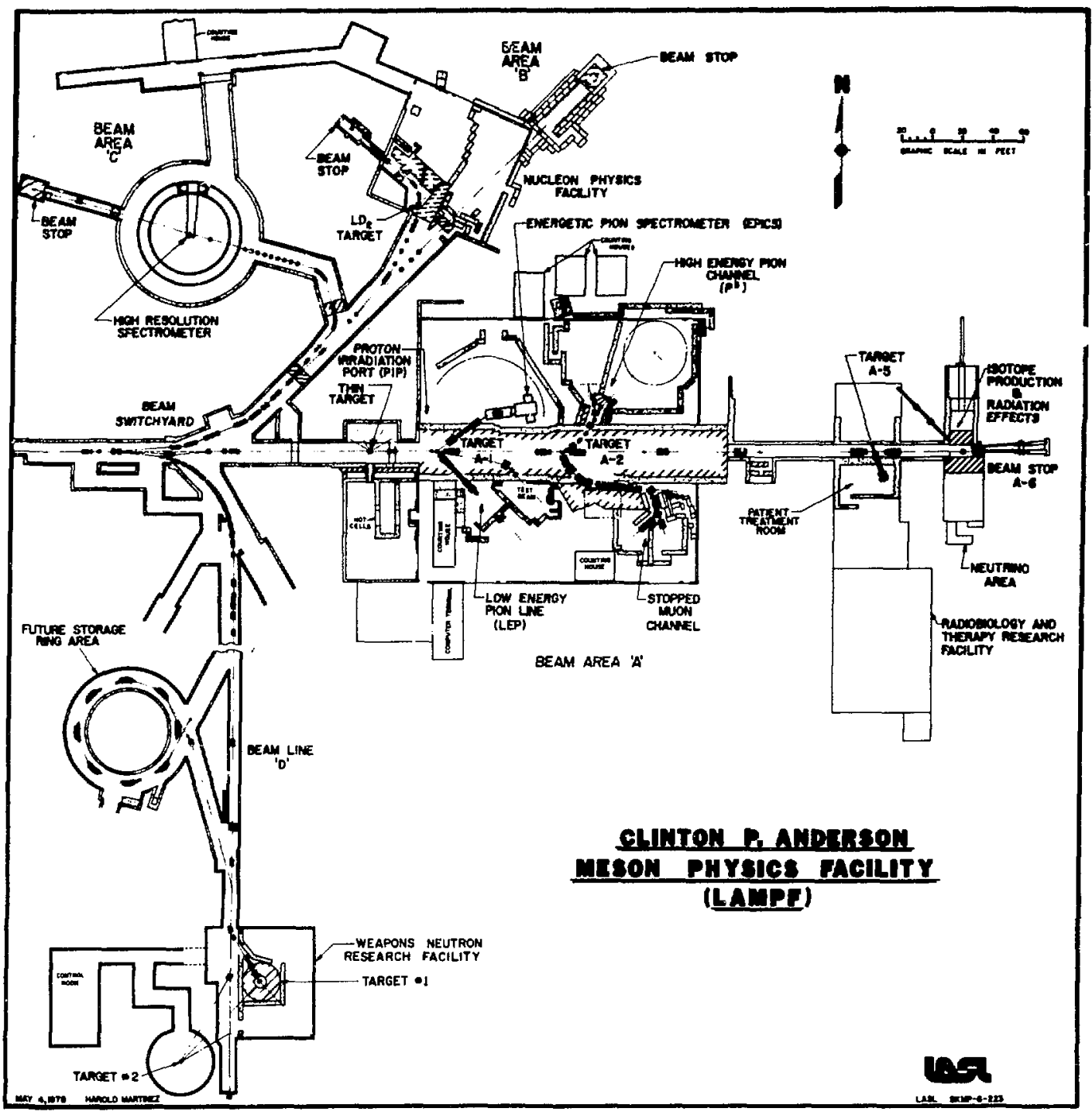

Fig. 18. LAMPF Experimental Area. 
plug, and the target must therefore be removed from the beam when access to the channel to desired. This target wos out of the beam about half the tine during all the tine that we were taking data.

The beam stop is copper and is water-cooled. It is a cylinder 8 in. In diameter and 50-in. 1ong. The 1sotope production experiments used targets placed directly in front of the beam stop, and much of our neutrino flux came from these targets rather than from the beam stop itself. The target holders, which were thicker than the targets themselves, were steel; each target plus holder was 4-cm thick. There were usually four of these targets in place.

The beam position and intensity are monitored by a series of wire profile monitors ${ }^{62}$ and toroidal current monitors. ${ }^{63}$ The profile monitors are multiwire counters placed in the beam; the secondary emission is monitored to give a profile of the beam. The toroids function as current transformers, the proton beam acting as a single-turn primary winding. They provide both analog and digital outputs of the beam intensity. We used the analog output as part of our triggering system, so we took data only when the beam was on. The digital output is proportional to the beam intensity in each macropulse; we scaled it as a measure of the total beam incident on the beam stop. A calibration pulse is provided continuously for calibrating the number of pulses per coulomb. This calibration was normally changed only when the beam Intensity changed (a history of the beam intensity is contained in the

62. E. W. Hoffman et al., Los Alamos Scientific Laboratory report LA-CK-79747 (1979).

63. Pau1 J. Tallerico, J. Vac. Sc1. Techno1., 12, 1200 (1975). 
final chapter); we checked the calibration weekly, and also when we had been notifled of a change.

\section{B. THE NEUTRINO HOUSE}

The neutrino house itself was opposite the beam stop, at $90^{\circ}$ to the beam direction. This minimized neutron-induced high energy photon events, since high-energy neutrons produced in the beam stop tended to go forward. There was $6.3 \mathrm{~m}$ of steel shielding in the direction of the beam stop (see the secton on backgrounds for the reason for this choice). The last $2.3 \mathrm{~m}$ mere not installed until after some background measurements had been made. Figure 19 shows the neutrino house and associated shfelding, and also the location. of our detector. There was, at the reduced shielding levels, a movable plug of shielding next to the beam stop; this plug was removed to yield a different shielding configuration quickly. The data taken with this plug removed proved difficult to interpret because the fluxes were very sensitive to the position of the beam stop (it could be moved about $60 \mathrm{~cm}$ along the beam direction). Because of this ambiguity of interpretation, the plug was removed and replaced with solid shielding aiter the fifth meter of steel had been installed. The east and west sides and the roof of the neutrino house were built of missile silo door counterweights, which were slabs of cast tron $6.1 \mathrm{~m}$ by $1 \mathrm{~m}$ by $30 \mathrm{~cm}$. The roof conststed of four layers of counterweights. The south wali was concrete with a stack of counterweights behind it. The floor was of concrete blocks.

Because of the problem with radioactive gas mentioned above In the section on the drift chambers), we attempted to completely seal the house, to keep the ga? out (this was not done, however, until March 1979). We then worried about a buildup of methane from the drift 


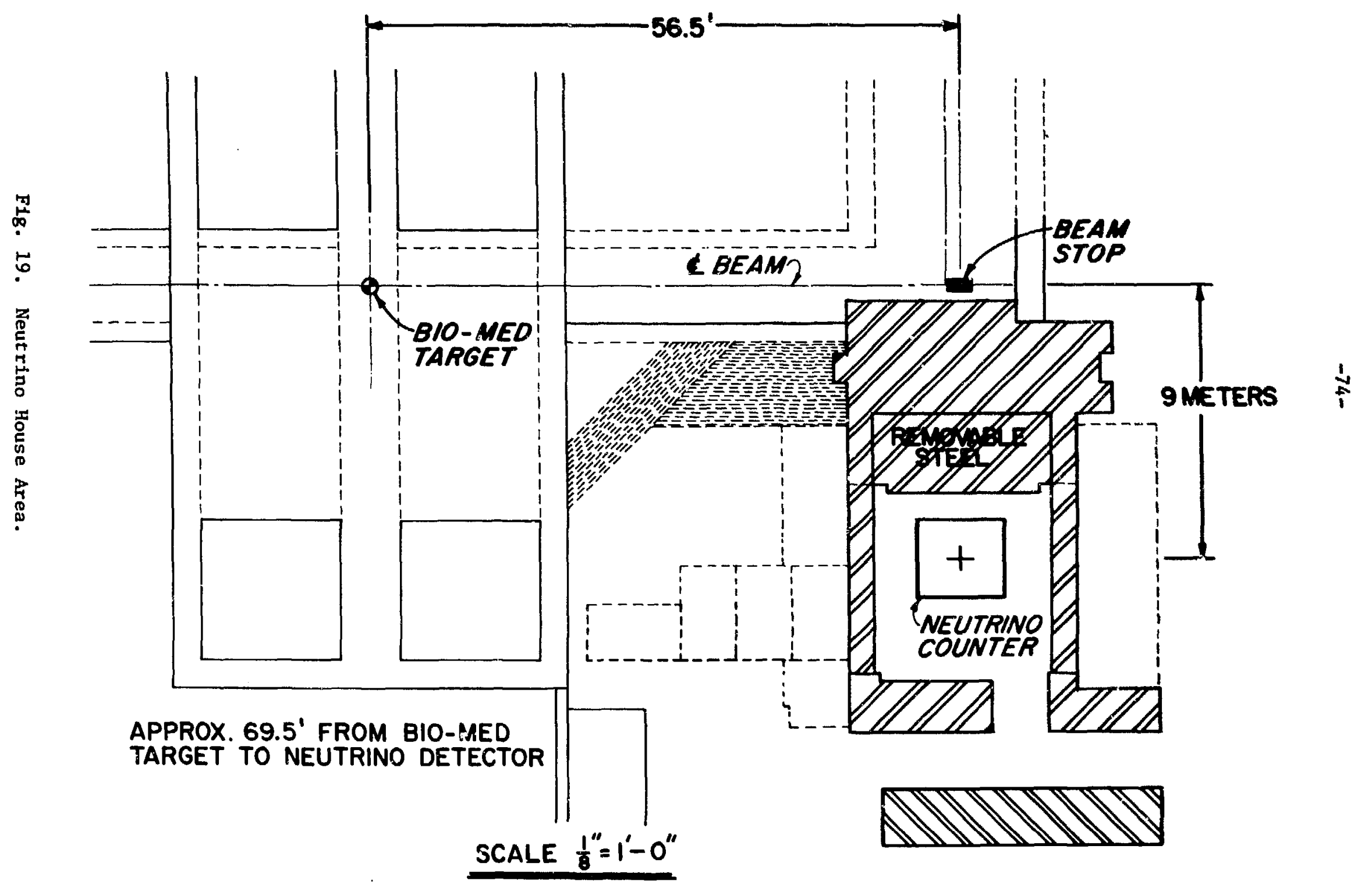




$$
-75-
$$

chamber exhauat, posalbly leadtug to an explosive axture. in exheart syoter was installed to peratt personnel access wen necesery. It turned out, horever, that the cesing was not tight, and wethane bulldup was not a problea. 


\section{CHAPTER 4}

\section{BACKGROUNDS}

There were two fundamentally different types of backgrounds for this experiment -- those which were beam--assoclated, and those which were not. Non-beam-assoclated backgrounds were the same whether the beam was on or not; they could therefore be measured between beam spills and subtracted. A low signal-to-nolse ratio sas tolerable with this type of background. Beam-1nduced backgrounds, on the other hund, could not be subtracted directly and attempts were therefore made to reduce these events to as small a fraction of the expected neutrino signal as possible. The cholce of a Cerenkov counter for the detector was made partly to reduce the backgrounds. The counter was not sensitive to recoll protons, as a scintillator or bubble chamber would have been.

\section{A. NON-BEAM-ASSOCIATED BACKGROUNDS}

The background events which were not assoclated with the beam came primarlly from cosmic rays, although tube noise also made a small contribation. The cosmic-ray anticolncidence shield was designed for the most efficient rejection of these events. Cosmic-ray-induced events In the energy range of $(20,60) \mathrm{MeV}$ were of the following types: charged events, Including delayed electrons from muon decay; gammas from muon bremsstrahlung; and gammas from muon decay with eiectron bremsstrahlung. We chose the energy region $(20,60) \mathrm{MeV}$ because this corresponded closely 
to the region over which we intended to analyze our data for actual neutrino-1nduced events. These events occur fron 0 to $60 \mathrm{Km}$, but the backgrounds were too high at the lower energlea for those data to be useful, and the neutrino spectrun fell off there enyway.

Charged events were vetoed priarily by the Inner layer of acintillators. The total charged cosic-ray rate in the $(20,60)$ weV window was $2.9 \times 10^{5} /$ Los Alamos day $(6 z$ of a 24-hour day, or 5184 seconds). Of these, $1.0 \times 10^{5}$ were muon jecags. Using the entre scintillator gystem with a $25-\mu s$ veto pulse, the residual rate in $(20,60)$ MeV was $2500 \pm 40 /$ LAday, a reduction of a factor of 100 . Most of these remaining events were true neutrals, rather than charged events missed by the scintillators, charged particles going through holes, or muon decays occuring after the gate. The inefficiency of the scintillator shield was monitored on-line using a coincidence between the bottom layer of scintillators $\left(\mathrm{A}_{6}\right)$ and a high-threshold (100-MeV) signal in the Cerenkov counter $\left(C_{H}\right)$. This provided a pure throughgoing muon trigger. An "or" of the other five sides of scintillators $\left(A_{1}, A_{2}, A_{3}, A_{4}, A_{5}\right)$ was then put in anticoincidence with $A_{6} \cdot C_{H}$. This inefficiency was usually near $2 \times 10^{-4}$. The $25-\mu s$ length of the veto pulse corresponded to 11.4 muon 11 fetimes, which gave a reduction in the number of mu-decay electrons of a factor of $10^{5}$. The inefficiency would therefore yield 20 events/LAday, assuming that we wer! measuring a real Ineffictency and not $\_$trigger inefficiency, and the gate would miss one mu-decay event/LAday. 
There vere also wone sall holea in the ocintillatoi shield, due to the way the scintillatora were stacked (see P18. 15). The contribution to the neutrals rate in $(20,60)$ Me vas measured by placing two sall scintillatorg over one of the holes, making a colncidence between thea, and asking how many of the neutralg came in colncidence wh that 1gnal. The result was that 20 events/LAday w111 come through those four holeg ca the top. There were similar holes on the sides, but the contribution from those was assumed to be negligible because the rate of near-hor izontal cosmlc rays 19 small compared to the vertical.

There ware two types of cosmlc-ray-induced neutral events which caused background events in the Cerenkov counter -- gammas from muon bremsstrahlung in the roof, and gammas from muon decay in the last section of the roof with electron bremsstrahlung. These two processes were 1ndependent and required different solutions; they w11 therefore be dealt with separately.

If the separation between the roof and the scintillator shield were large enough, a muon and a wide-angle bremsstrahlung gamma could become widely enough separated so the gamma entered the Cerenkov counter but the muon completely missed the scint1llators (see FIg. 20). The gamma would then convert in the water and cause a neutral event. The solution to tils problem was to effectively bring the roof very close to the counter, which was done by installing lead directly outside the scintillators (see F1g. 13). The desired thickness of lead was measured by first installing $1.8 \mathrm{~cm}$ of lead on the sides and then measuring the effect on the neutrals rate of no lead, $1.8 \mathrm{~cm}$ of lead, and $3.7 \mathrm{~cm}$ of lead on the top. From these measurements, we concluded that the attenuation length of gammas in lead $1 \mathrm{~s} 10.8 \mathrm{~g} / \mathrm{cm}^{2}( \pm 5 \%)$, and that the 


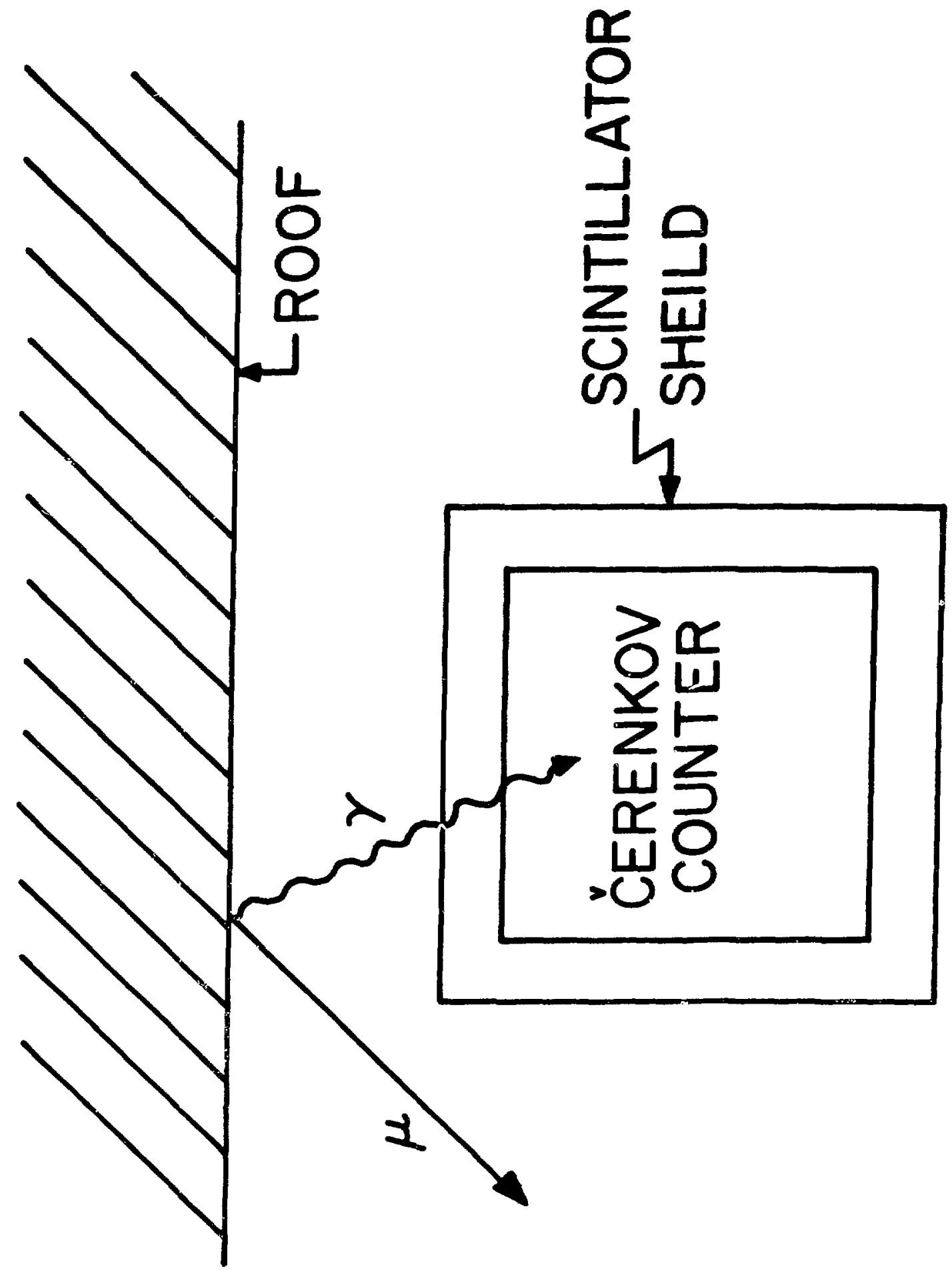

F1g. 20, Muon brensstrah'ung. 
3.7 ca of lead reducad the neutrals evente coning from mon bremetrahlung through the top of the counter by a factor of 50. The muon bremetrahlung sate through the top was $20 /$ Laday with $3.7 \mathrm{~cm}$ of 1ead. Subsequently, another $1.2 \mathrm{~cm}$ of lead was installed on the ides of the counter, alking a total of $3.0 \mathrm{~cm}$ on the sides. The rate of neutrals in $(20,60)$ MeV with this amount of lead was 650 eventa/LAday.

The other mor contribution to the cosicic ray-assoclated neutral events came from wons which stopped in the lead, decayed, and gave a bremgtrahlung Bamma frow the decay electron (see F1g. 21). Even if the electron did not escape from the lead to produce a pulse in the scintillators, the gamma could go into the counter and convert, causing a neutral event. Many of thege events were eliminated by Ingtalling detectors outside the lead, which detected the muon as it entered the lead and thus provided a veto. We used scintillators on the top and drift chambers on the sides (see F18. 17). We did not use timing or position Information from the drift chambers, but simply treated them as if they were scintiliators. W1th a $25-\mu s$ veto pulse for the scintillators and 11 us for the drift chambers, the rate of neutrals in $(20,60) \mathrm{MeV}$ was 450 events/LAday. Th1s was less of an effect than we had hoped for, and we decided that most of the residual events could st11l be of the muon bremsstrahlung type. The Cerenkov counter was quite close to the north, west, and east walls of the neutrino house, but it was not so clcse to the south wall (see F1g. 19). We decided to try ralsing the south drift chamber at an angle -- it would still catch muons entering the lead, since they were mostly vertical, but it rould also catch more wide-angle muons which might be associated with bremsstrahlung gammas in the counter (see F1g. 22). W1th the south 


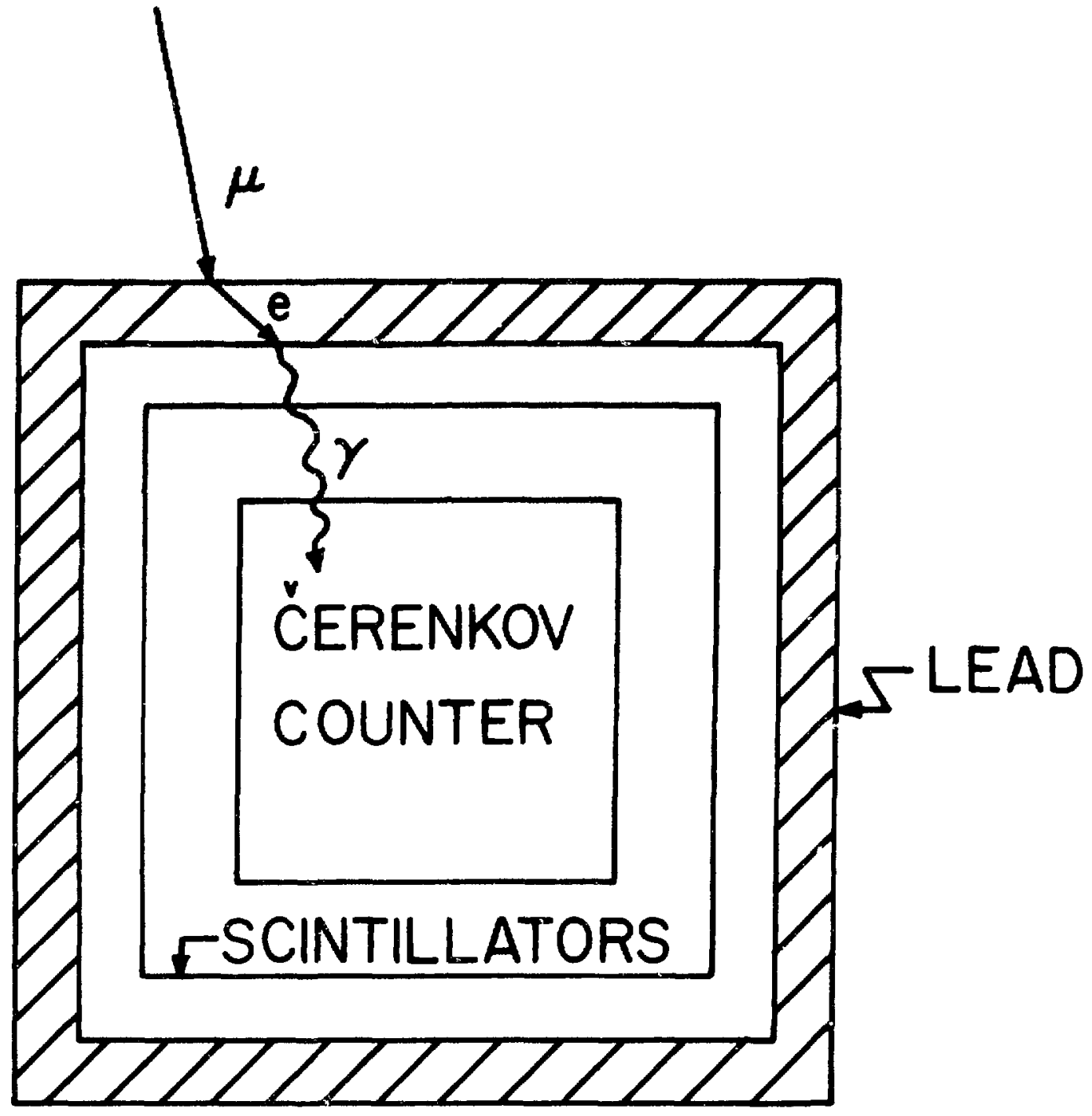

Fig. 21. Muon decay with

electron bremsstrah-

lung. 


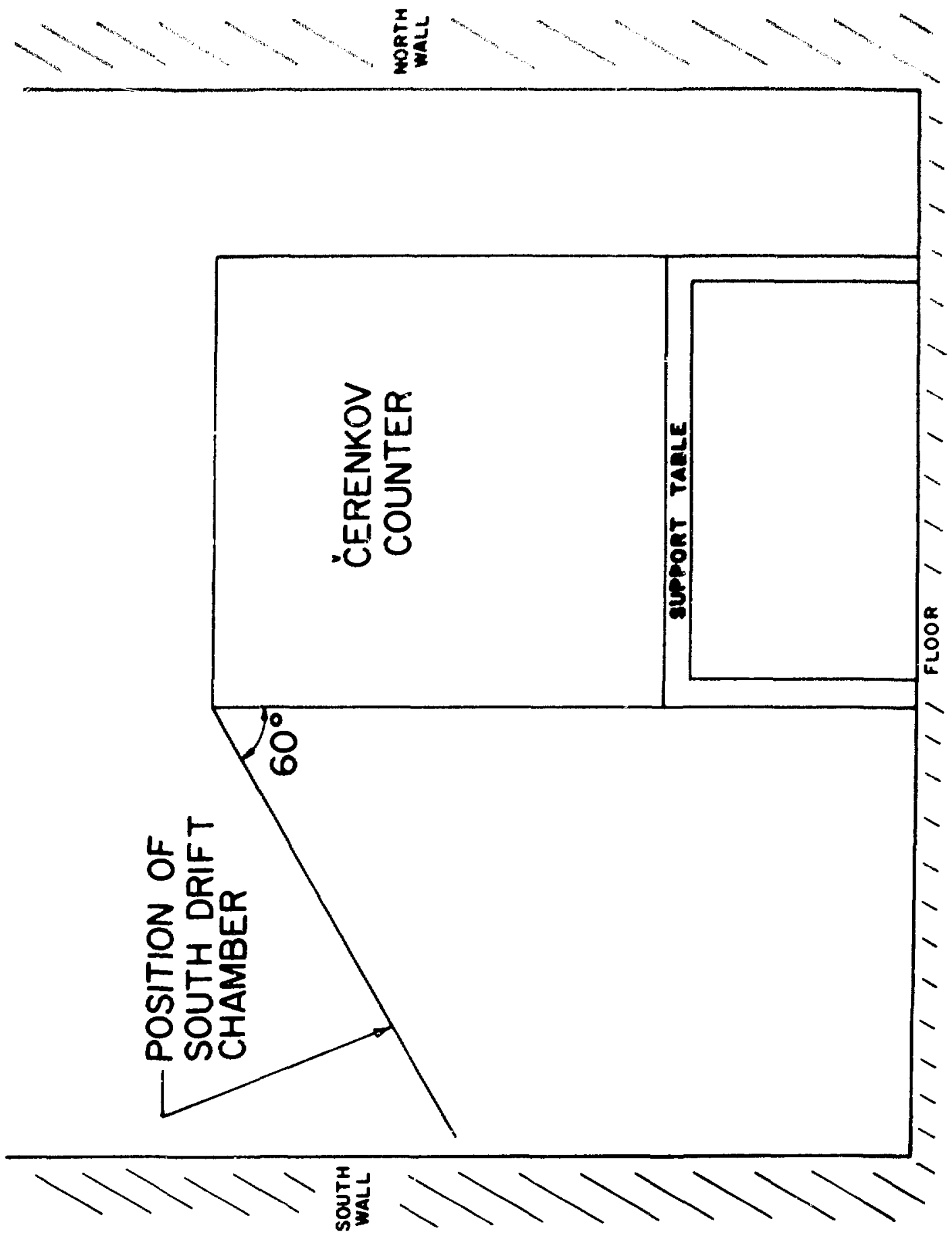

Fig. 22. Position of south drift chamber. 
drift chamber in this position, the neutrale rate we 325hadey. Indicuting that our guess bout wde-angle mont was correct. We left the chaber in this position for all further running. Figure 23 sowe the spectrum shape of the cosmic-tay events with no ant1, the ahort ant1, the long ant1 with ocintillators only, and the total ant1. Ml rates are per Los Alamos day.

A check was made to see that all of our neutrals events vere muon-assoclated. For a short time, an extra fool, of steel was added to the roof of the neutrino house. The ratio of the total cosalc-ray flux In the counter with the thlcker roof vs the normsl roof was 0.926 , whereas the ratio of neutral events was $0.913 \pm 0.012$. Fron this we concluded that thert was no evidence for a component of the neutrala events with an attenuation in steel different from that of the muons. Cosmic rays do have both electronic and hadronic components as well as the muonis; our roof eliminated the electrontc part. The Cerenkov counter was not sensitive to nucleons, except those at very high energy; this was the component that the above test would have shown if ir were present, since nucleons would attenuate 1 i the steel much faster than the muons.

Tube nolse could also look like a neutral event, since it would not be associated with a signal in the anticolncidence shield. One way to get an 1dea of whether this was a problem was to reduce the Intrinsic gain of the councer, so the tube nolse corresponded to a higher energy, and see if the neutrals rate increased. Th1s was done by looking at the counter after it had fust been filled, before the wavelength shifter was added. The gain was low by a factor of 2.6 compared to later, when the wavelength shifter had fust been added. We looked in $(30,60) \mathrm{MeV}$, and 


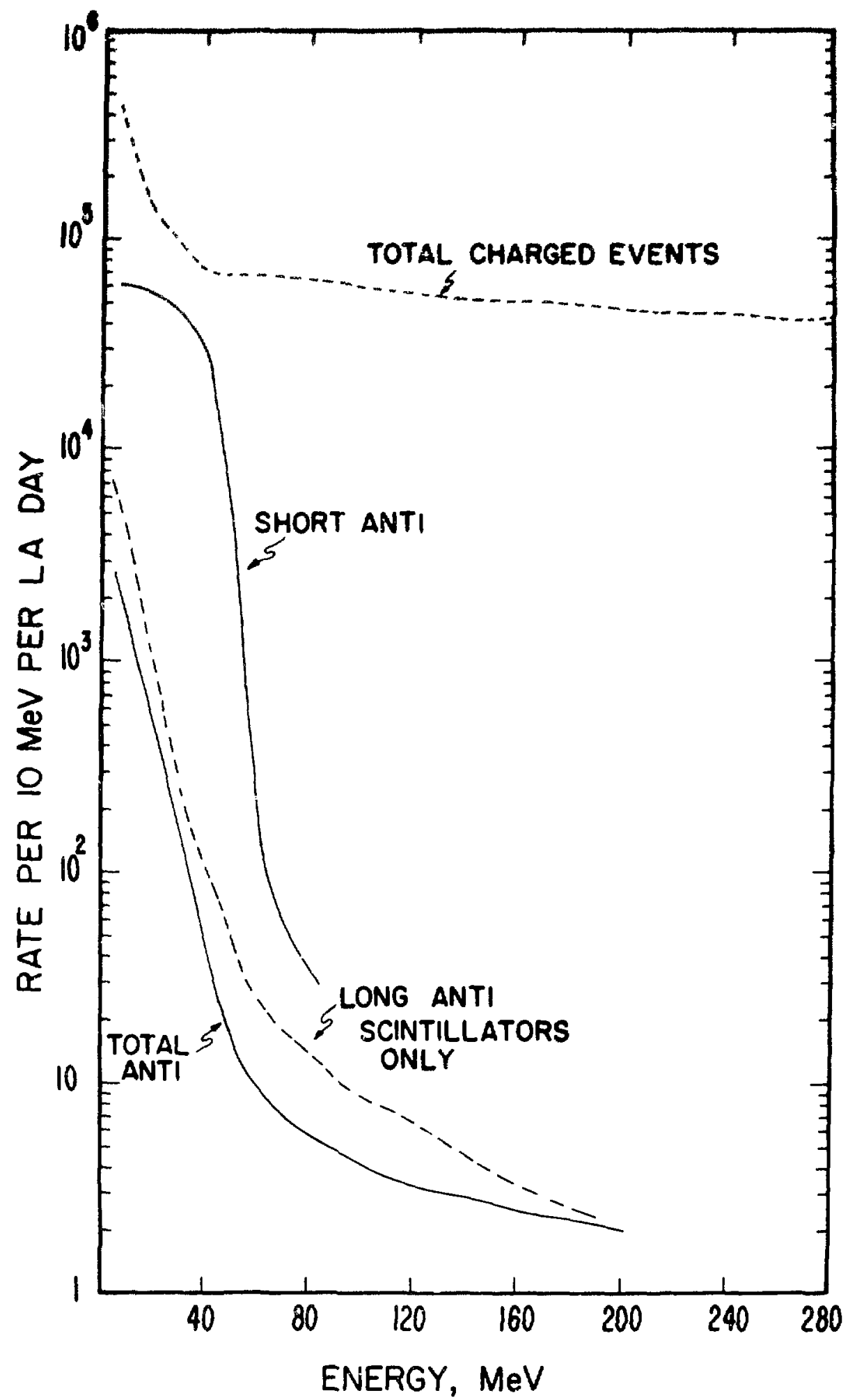

F1g. 23. Spect rum shape of cosmic-ray backgrounds. 
found, wth no wavelength ohifter, that, the neutral, rate was 325 haday. After the wavelength shifter was added and the gain readjusted; the neutrals rate in $(30,05 \%$ KeV was $100 /$ hadsy. We were, therefore, not far from the tube notse and 1rdeed wight st111 have contribution to it even at the higher gain. Ceriainly if the gain of the counter dropped because of clouding of the water, for instance, the tube notse contribution would increase.

Backgrounds with the $\mathrm{D}_{2} \mathrm{O}$ f 111 of the counter were messured only with the full ant 1 system in place and operational. They were the same as the $H_{2} \mathrm{O} 111 \mathrm{~s}, 350$ events/LAday in $(20,60) \mathrm{MeV}$. Th1s rate, through all our running, fluctuated by about $25 \%$, which we assumed was due to fluctuations in the primary cosmlc-ray flux, changes in tube nolse, and minor electronlcs problems.

\section{B. BEAM-ASSOCIATED BACKGROUNDS}

Beam-assoctated backgrounds in the Cerenkov counter were of several types. In principle, these were more insidious than non-beam-associated backgrounds, since they could not be subtracted on line. Thermal neutrons, which gave capture gamma rays, were numerous but low in energy; they got into the energy region which was interesting for neutrinos only through resolution and pileup effects. They could also contribute to the dead time of the anticoincidence shield. High-energy beam-assoclated neutral events have also been seen in the counter. These were probably due to neutral pions created by high-energy neutrons; the pion decay would produce two high-energy gammas, which could convert in the counter. These backgrounds were measured by running at a serles of different shielding conditions, and the final 


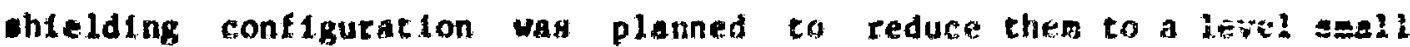
compared to our expected sensitivity. The thick (8-cs-graphite) blomedical target Jumt upstream of the beam stop created large amounts of background when $1 \mathrm{t}$ was in the beam. The shiclding in that direction was made as thick as posalble, but there was $8+111$ a considerable Increane in background compared to when that target was out. There was also undoubtedly an increased electron antineutrino flux due to negative-p1on decay in flight downstream of that target, but that should have been a small effect because of the small solid angle. There was also material other than hydrogen inside our scintillator shield: another source of background was from reactions of electron neutrinos from normal muon decay on these other materlals, princlpally carbon and oxygen. Differentlal shielding measurements were not done on $\mathrm{D}_{2} \mathrm{O}$, but the total beam-assoclated rate at low energles (where the thermal neutrons predominate) was measured and compared to the $\mathrm{H}_{2} \mathrm{O}$ data.

The fall-off of thermal neutron events in the Cerenkov counter was planned to be $10^{7}$ from 0 to $20 \mathrm{MeV}$. Figures 24 and 25 show this to have been true for both blomed target in and out, although the absolute rate was higher by about a factor of 35 for blomed target in (see Table VI). The assumption here was that all the low-energy events in the subtracted spectrum were thermal neutrons. Flgures 26 and 27 show the measured thermal neutron flux, assuming a continued exponential falloff, and the predicted neutrino spectrum for $R=1.0$. From this we see that above $25 \mathrm{MeV}$ the thermal neutrons did not contribute. 


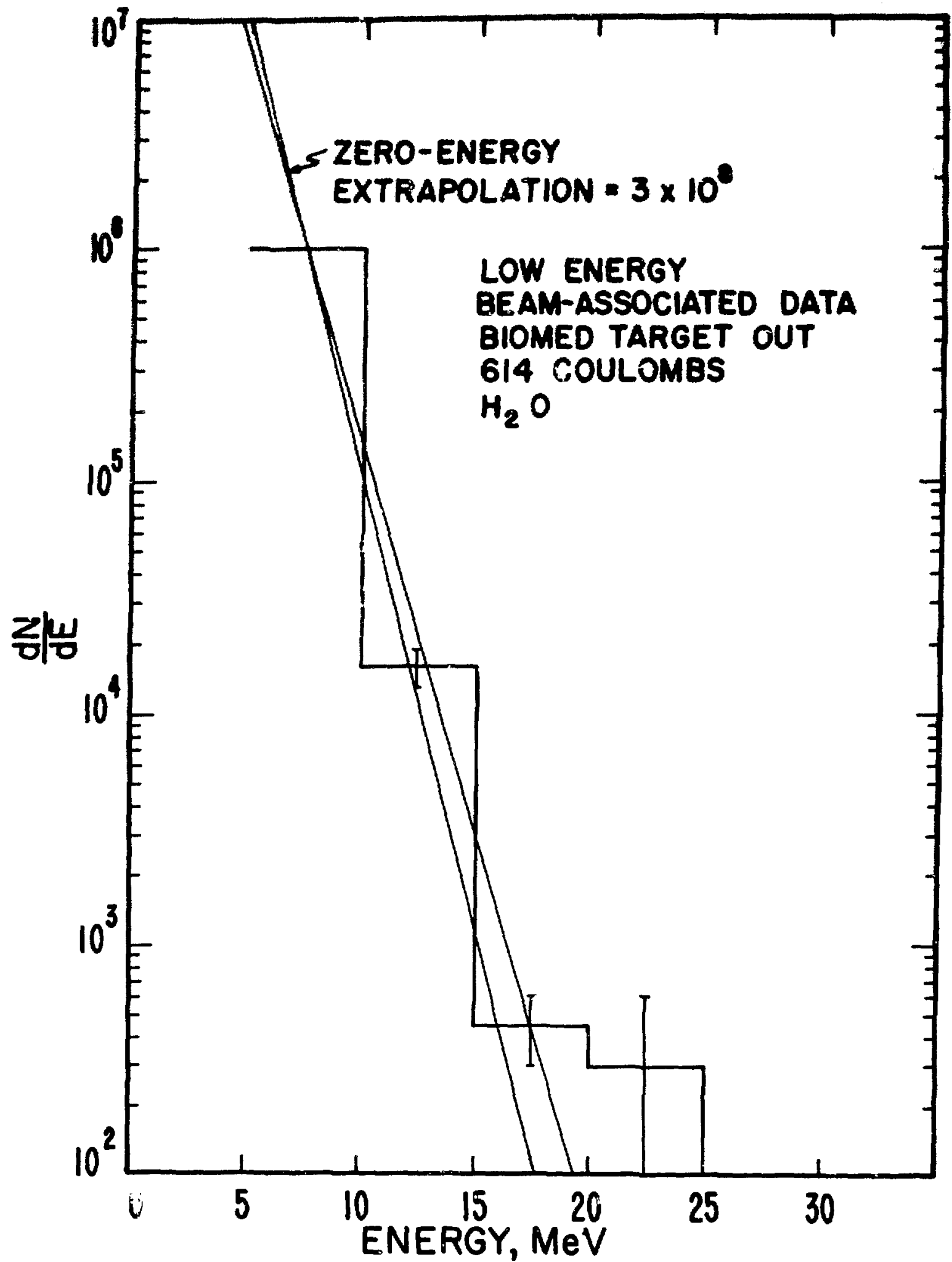

Fig. 24. Therma1 neutrons, Biomed target out, $\mathrm{H}_{2} \mathrm{O}$. 


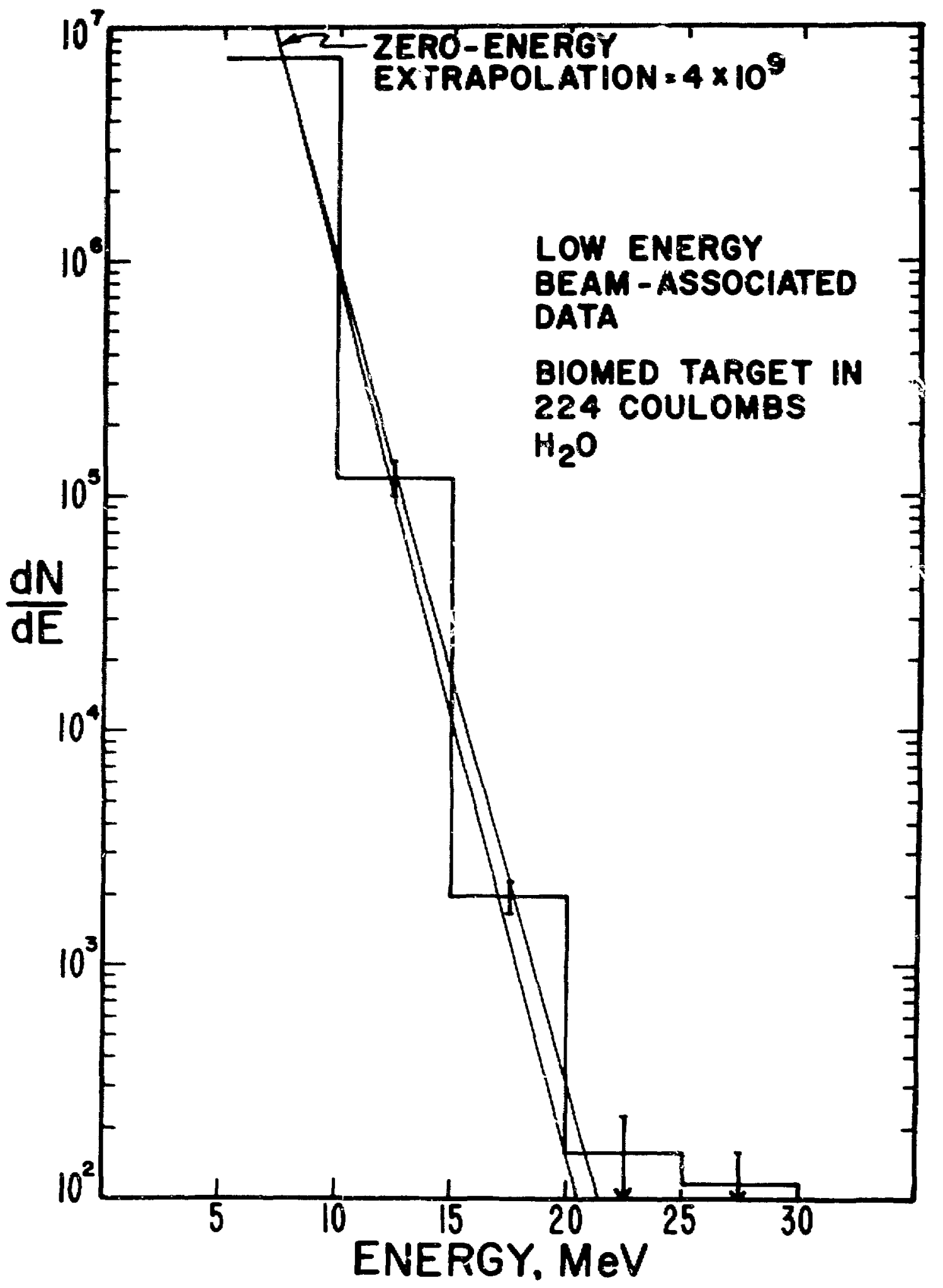

Fig. 25. Thernal neutrons,

Biomed target in, $\mathrm{H}_{2} \mathrm{O}$.

Sutmed data, July 1977-March 1978. 


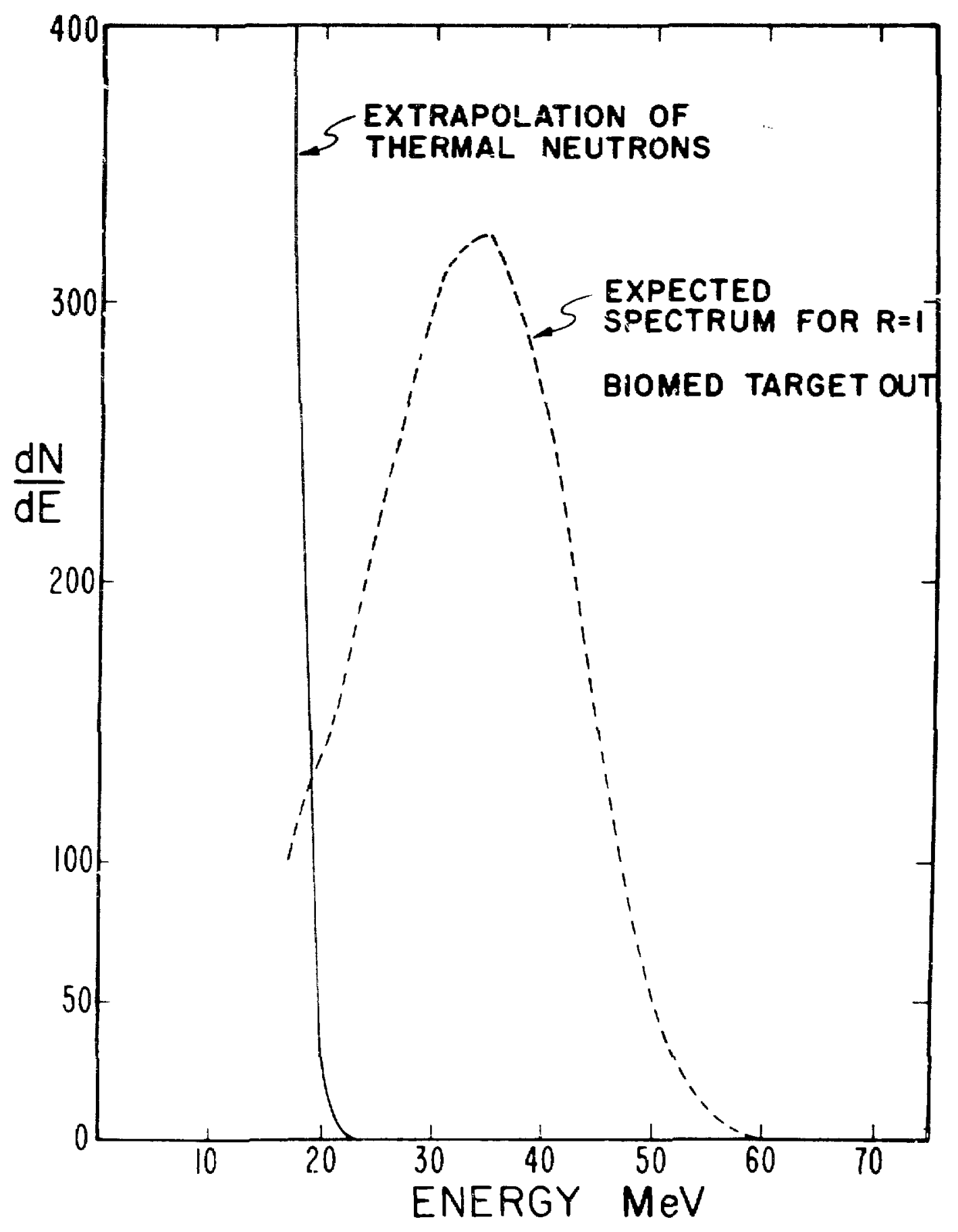

Fig. 26. Thermal neutrons, Biomed target out, $\mathrm{H}_{2} \mathrm{O}$, with calculated reutrino spectrum.

All expected sportra have $10 \%$ error. 


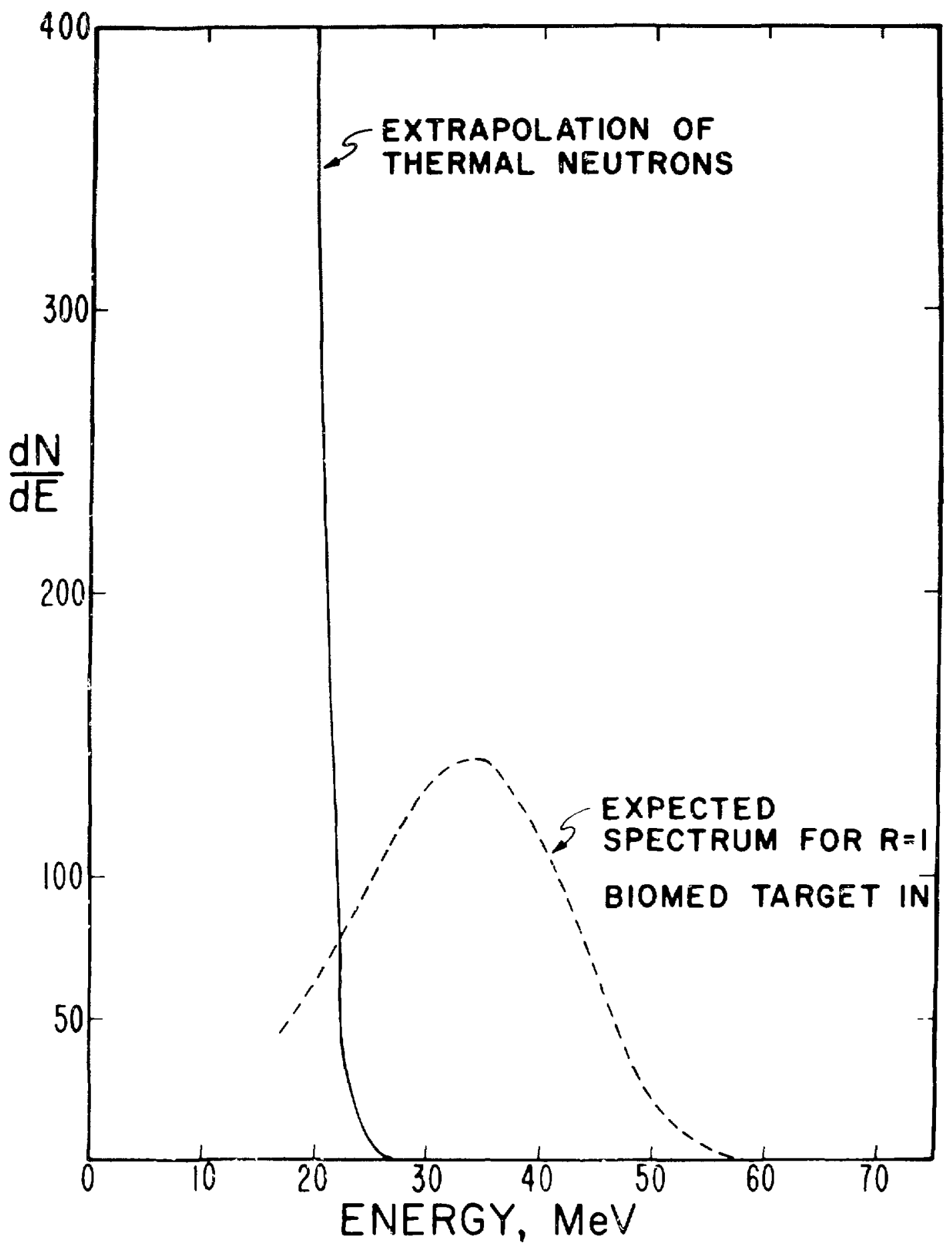

Fig. 27. Thermal neutrons, Biomed target in, $\mathrm{H}_{2} \mathrm{O}$, with calculated neutrino spectrum. 
High-energy neutral events fell off wuch wore olowly with energ; 1t was necessazy to attenuate them by installing a sufficient anount of shlelding in the direction of the beam stop to reduce the number of events in $(20,60)$ MeV to about one per day at full bean intensity. The last sections of shielding, as Indicated In F1g. 19, were not In place when the apparatus was originally installed. This allowed us to make beam-associated background measurements with the low bean intensity then avallable, at two shielding configurations other than the final one, namely $4 \mathrm{~m}$ and 5 m of iron shielding. Figure 28 shows the results of these measurements, plotting neutral events in $(20,60) \mathrm{MeV} /$ Coulomb vs shielding. The final shielding was $6.3 \mathrm{~m}$ of steel, which corresponded to less than about 2 events per day at $300 \mu \mathrm{A}$ of beam on the beam 8 top. This is to be compared to the expected neutrino event rate of about 70 events per day, for $R=1$. The shape of the background at 5 m of shielding is shown in Fig. 29. This shape was found to be the same at $4 \mathrm{~m}$ and $5 \mathrm{~m}$ of shielding, Justifying the exponential extrapolation to higher shielding thicknesses. This background, extrapolated to $6.3 \mathrm{~m}$ and wh the shape shown, was subtracted from the data, as described in the final chapter.

The blomedical target was also a source of neutron backgrounds. Figure 19 shows the relative position of that target to the beam stop and the neutrino detector. The angle between that target and the detector is quite forward, which contributed to the enhancement of the neutrons. The observed backgrounds were significantly higher with the target in than out at energies up to $20 \mathrm{MeV}$, as shown in Table VI. The target-in data, in fact, show a nonzero background up to $30 \mathrm{MeV}$.

The state of the biomed target was recorded on-1ine with each 


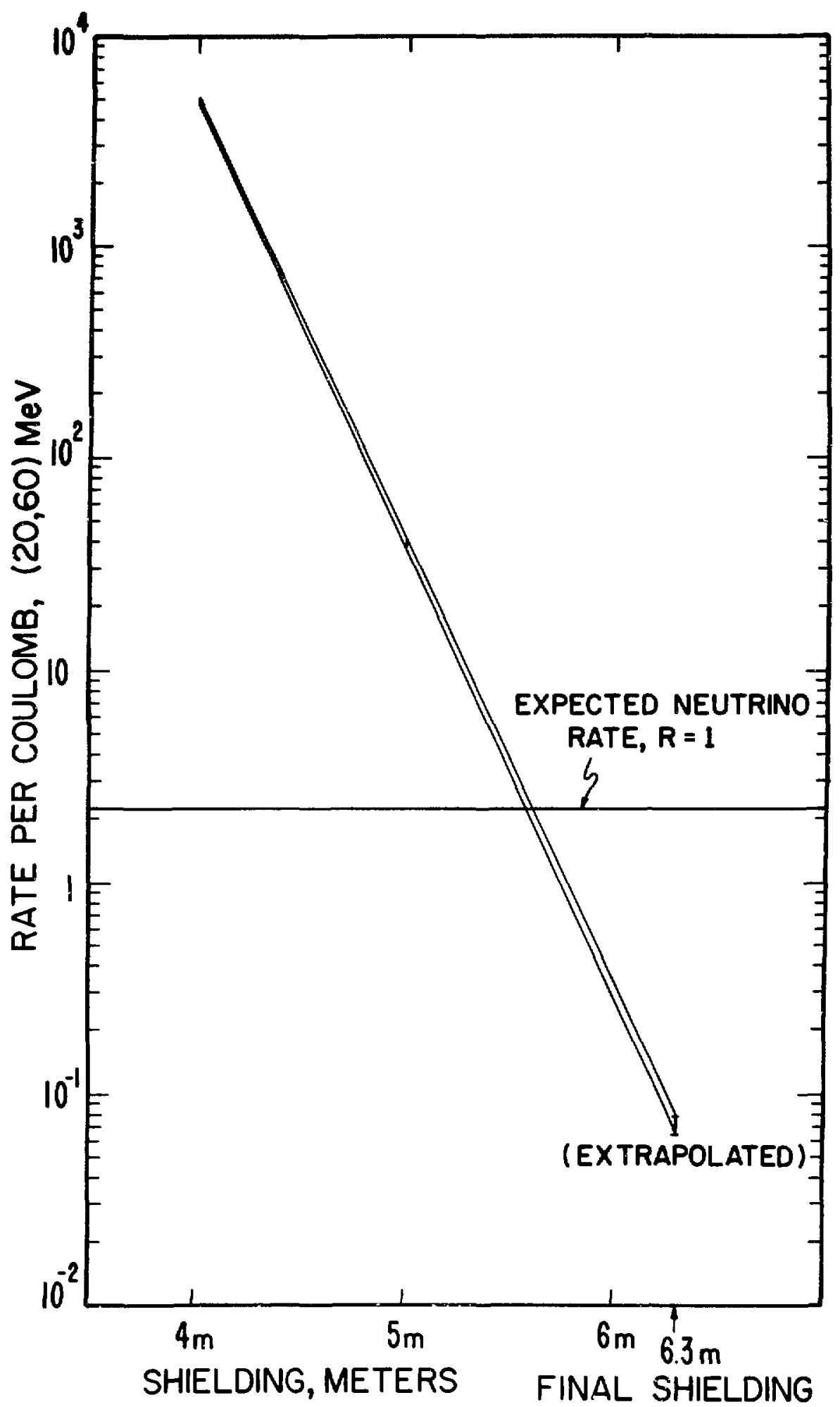

Fig. 28. Beam-associated 


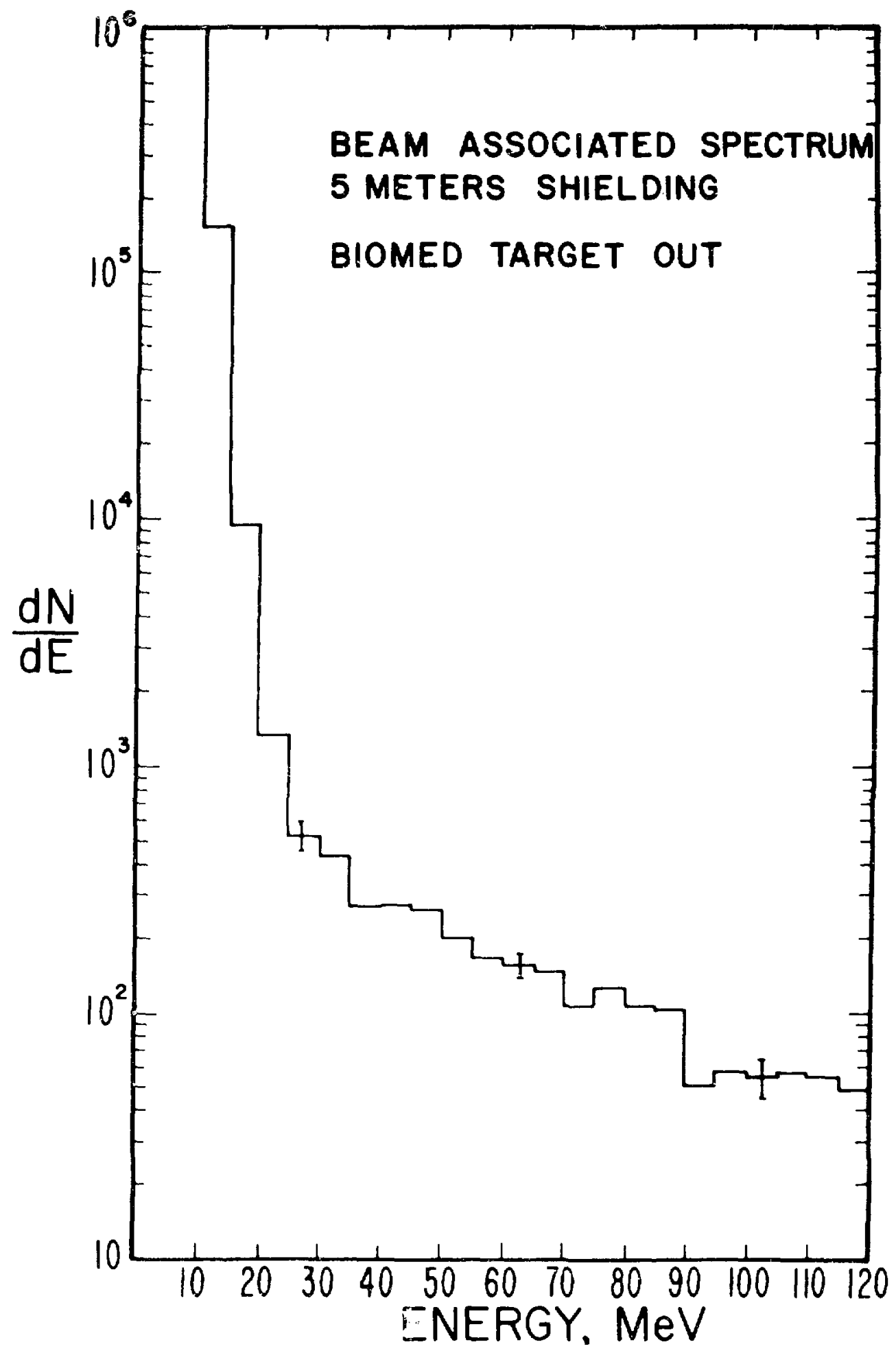

Fig. 29. Spectrum shape, beamassociated neutrals, 5-m shielding, Biomed target out. 
TABLE VI

RATIO OF NEUTRON BACKGROUNDS, BIOMED TARGET IN/TARGET OUT, AS A FUNCTION OF ENERGY

\author{
Energy, MeV \\ 0 (extrapolated) \\ 5-10 \\ 10-1:
}

$15-20$
R, In/Out (Neutrals Only)

35

20

15

10

event, and the two sets of data were treated completely separately in al1 analysis.

Cross-sections of electron neutrinos on carbon, oxygen, and aluminum have been calculated for the energy region $0-53 \mathrm{MeV}$, as discussed in the theoretical section. These cross sections are much suppressed due to nuclear effects and contributed very little to the background. Figure 30 shows the relative cross sections as a function of energy of $\bar{v}_{e} p, v_{e} d, v_{e}{ }^{12} \mathrm{C}$, and $v_{e}{ }^{16} 0$. Assuming $5800 \mathrm{~kg}$ of oxygen, $500 \mathrm{~kg}$ of carbon, and $100 \mathrm{~kg}$ of aluminum, the electron-neutrino reactions would contribute 0.06 events/Coulomb to the observed electron antineutrino signal over all energies.

The cross sections for carbon and oxygen were put through the Monte Carlo calculation mentioned above, to include the effects of radiation 1osses, resolution, and edge effects. The resulting spectra were then subtracted from the final spectrum, as described in the conclusions. The cross section as a function of energy for aluminum is not known, but the amount of aluminum was relatively small. The energy spectrum was assumed to be the same as that for oxygen. 


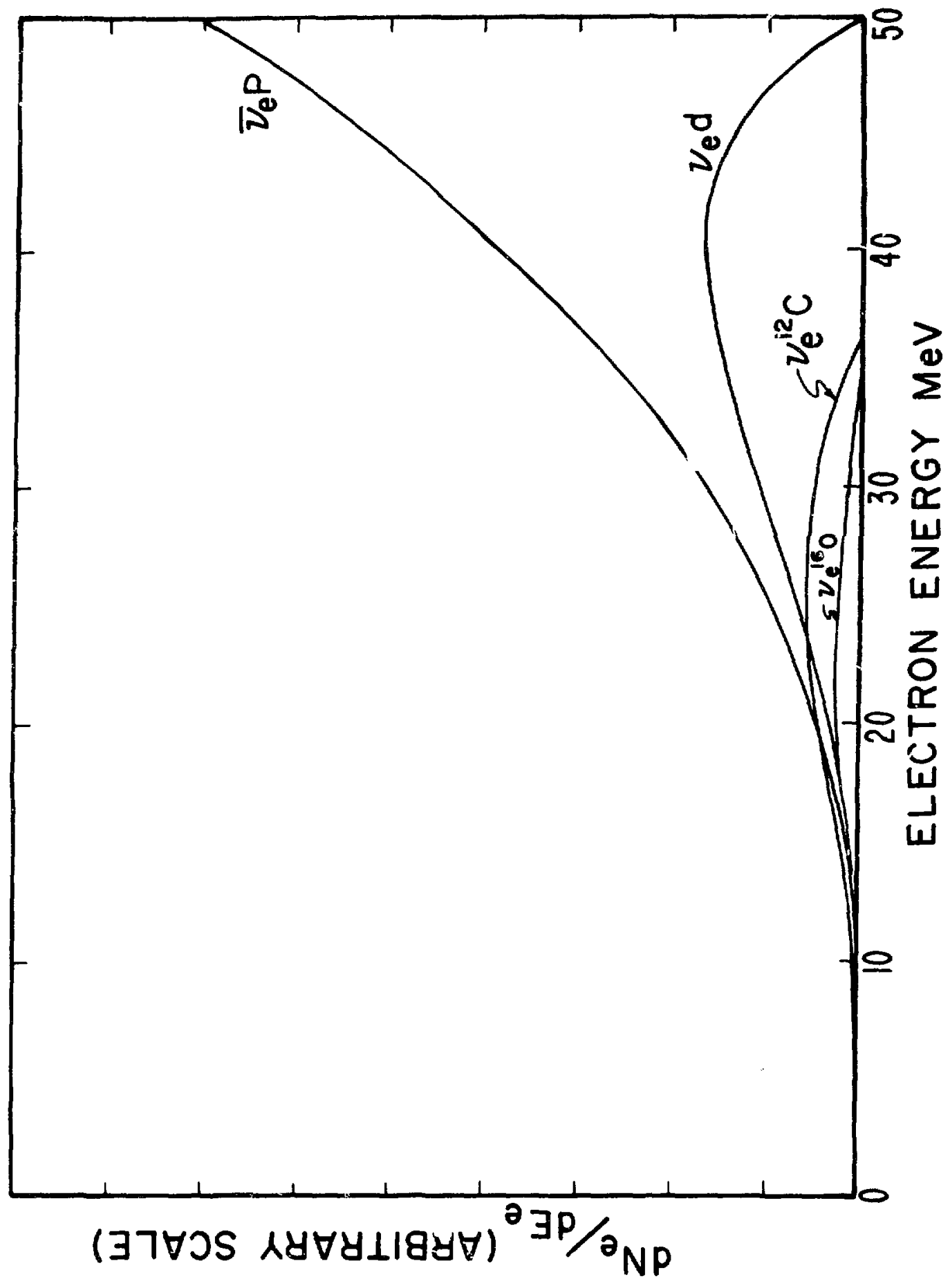

Fig. 30. Relative cross sections of $\bar{v}_{e p}, v_{e} d, v_{e}{ }^{12} c$, and $v_{e}{ }^{16} 0$ as a function of energy. 
Differential shfelding measurements were not done during the $D_{2} O$ f111, but the effect on the thermal neutrons could be seen by looking at the 1ow-energy portion of the subtracted spectrum. Figures 31 and 32 correspund to $\mathrm{F} 1 \mathrm{gs} .25$ and 26 for $\mathrm{H}_{2} \mathrm{O}$; 1 th can be seen that the zero-energy extrapolated rate was lower in $\mathrm{D}_{2} \mathrm{O}$ by a factor of 4 for both blomed target conditions, giving a ratio, $\mathbf{i r} /$ out, of 35 , as before. From this we can conclude that, since the thermal neutron capture cross section 1s lower In $\mathrm{D}_{2} \mathrm{O}$ by a factor of 550,64 not all of the zero-energy extrapolation consisted of thermal neutron captures in water.

64. Reactor Handbook, Vol. I, ed. C.R.Tipton, Jr., New York, Interscience (1960). pp.848-850. 


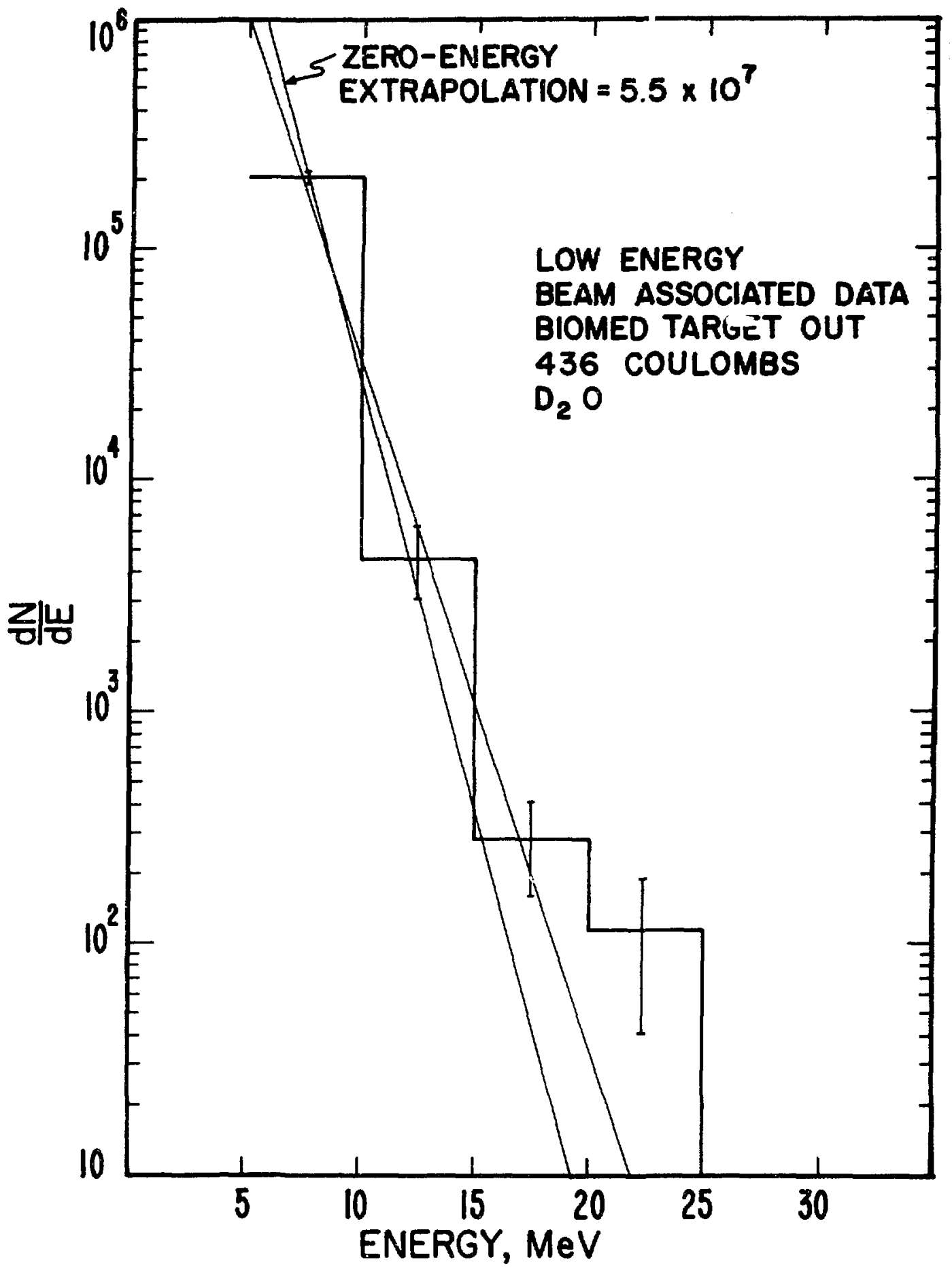

Fig. 31. Thermal neutrons, Biomed target out, $\mathrm{D}_{2} \mathrm{O}$.

Summed data, June-September 1978. 


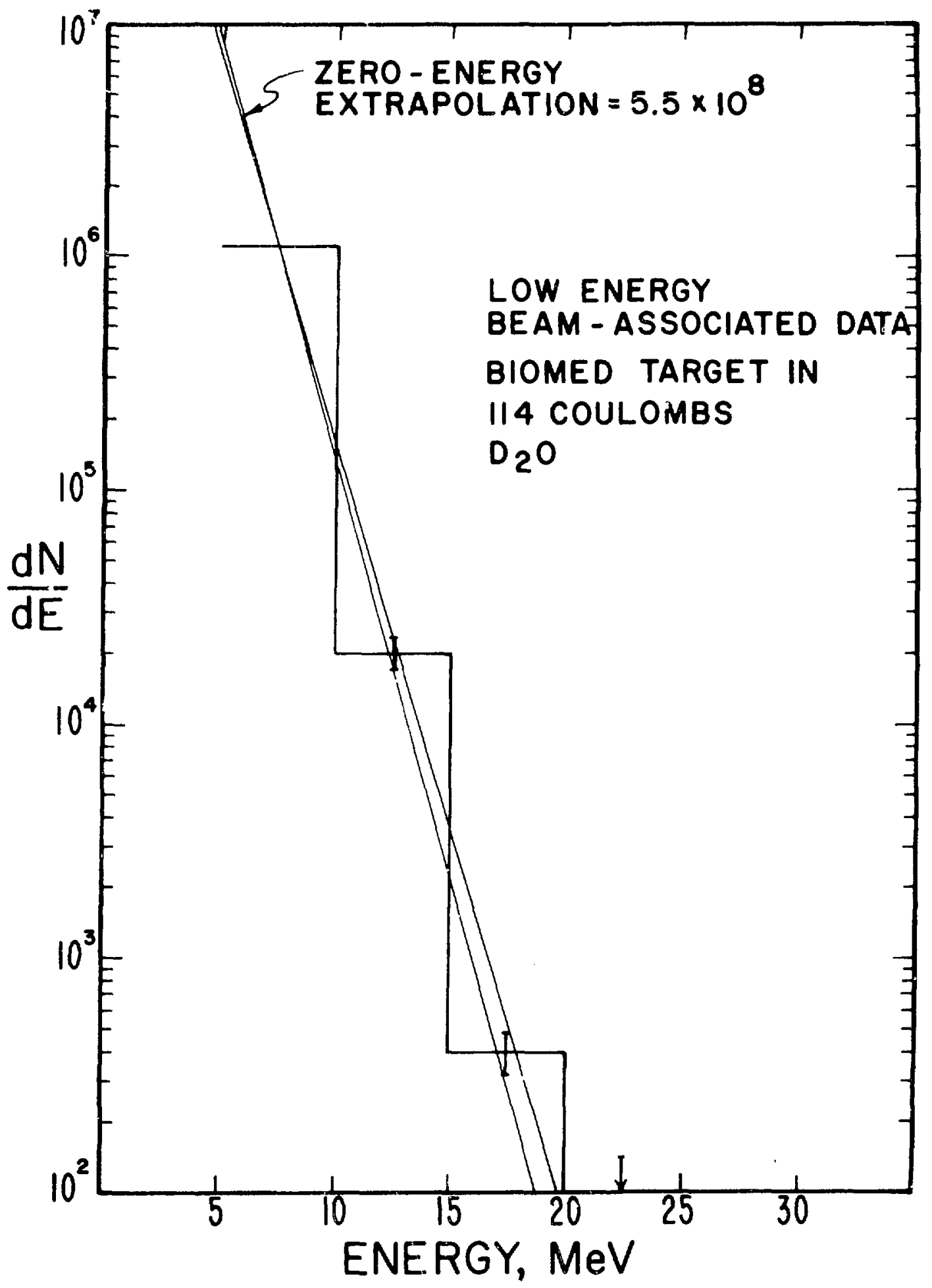

Fig. 32. Thermal neutrons,

Blomed target in, $\mathrm{D}_{2} \mathrm{O}$.

Summed data, June-July 1978. 
CHAPTER 5

ELECTRONICS

A block diagram of the event definition logic for the experiment appears in Fig. 33. Briefly, the electronics provided event definition and an event trigger to the computer for neutral events (neutrino candidates) and several other types of events, which were used for gain calibratiou and subtraction checks.

The phototube signals were bussed together in groups of four at the Cerenkov counter, giving us 24 signals in all, which we referred to as "supertubes." The supertube signals went directly to a CAMAC discriminator coincldence register, which was triggered by the event trigger and which gave a 1 for every supertube whose signal was above the discrimfnator threshold, and a 0 for the signals which were below -24 channels in a11. This was read in for each event. The gains were set so that half the discriminators were set for a typical muon decay event. The supertube signals also went into six 4-fold 11near mixers, the outputs of which were signais for a total side. The six side signals then went directly to stx 256-channel CAMAC ADCs, which were read in by the computer for each event; these side signals also went to a voter colncidence (preceded by discriminators), which we typlcally ran at $2 / 6$, to reduce contributions from tube nolse. Finally, the side signals went into a linear mixer and fanout to provide a total signal. This total signal went through a variable attenuator, to allow for fine 


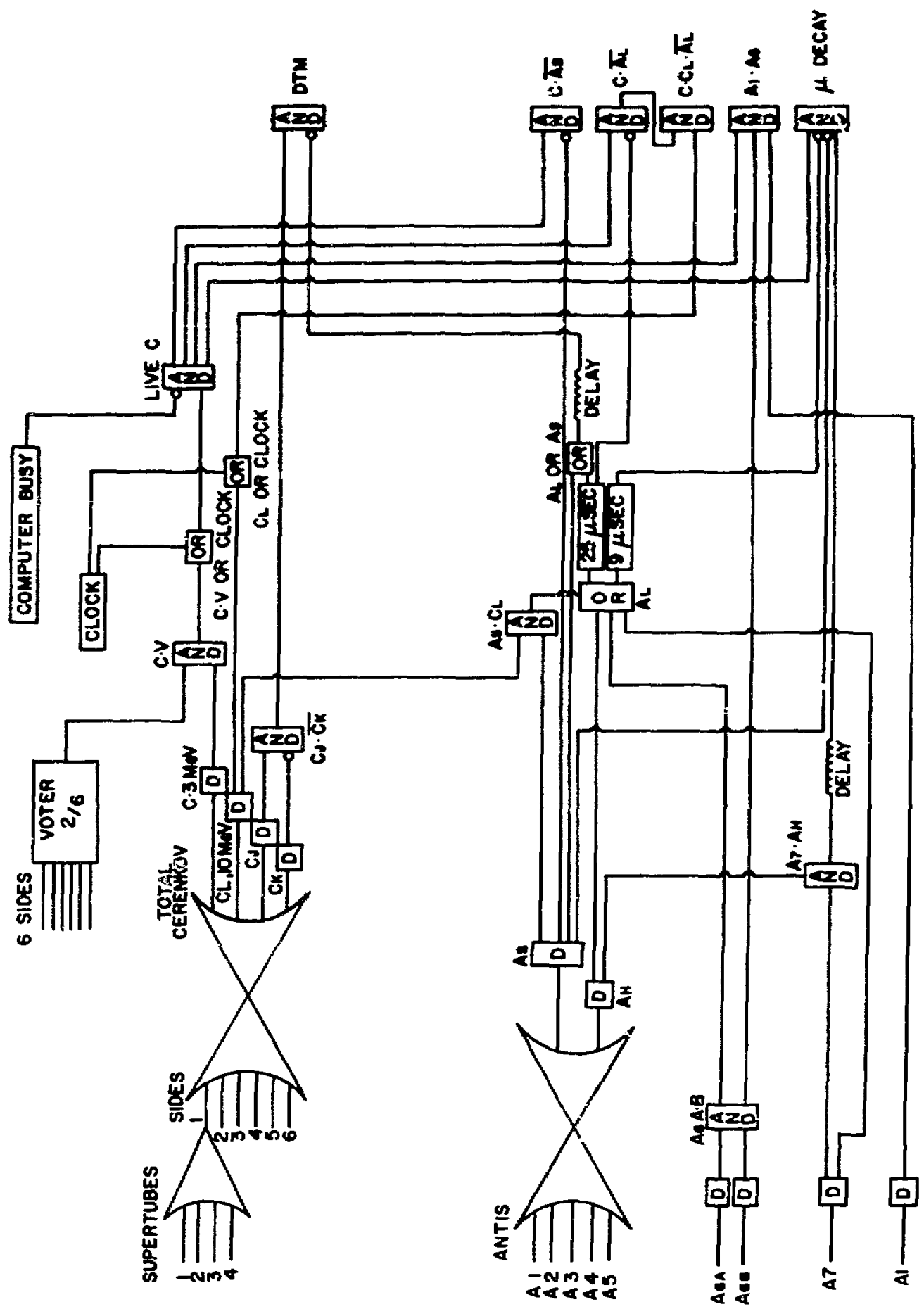

Fig. 33. Electronics block diagram. 
adjustiwents of the gain, and then Into two 256-chamel GaMc ADCs, ose at $1 \mathrm{MeV} / \mathrm{channel}$, and the other at $5 \mathrm{MeV} / \mathrm{channel}$. These were also read in for each event. The total signal \&lso went through several discrininators for event definition, as follows: C, 3-HeV, low-level threshold; $C_{L}, 10 \mathrm{MeV}$, the lower threshold for neutrino candidate events; $C_{M}, 30 \mathrm{MeV}$, or $1 \mathrm{gins} 11 \mathrm{y}$ used as an upper level for stopped mons;

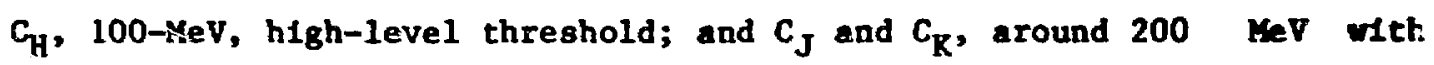
$C_{J}$ slightiy less than $C_{X}$, for dead-tive wontor events.

The signals from all eight tubeg on a side of scintillators vere bussr:d together at the scintilletors, and a total signal for each side was brought into the trailer. The exception to this was the bottom scintillator, $A_{6}$, where the four tubes on each end were bussed together, and a coincidence made between the two sigaals (labeled $A_{6} a$ and $A_{6} b$ ) to reduce nolse. Th1s was necessary because we had used the nolstest tubes on the bottow; very few (about 40/LAday) neutral events came through the bottom and nowhere else, so the efficiency did not need to be high. Scintillators 1-5 went through a linear mixer and then into two discriminators, $A_{S}$ (short), $300 \mathrm{~ns}$ and high rate, and $A_{B}$ (high), at a somewhat lower rat. The purpose of $A_{S}$ was to discrimlnate against prompt charged particles; a high rate could be tolerated here because of its short length. The higher threshold, $A_{H}$, was a primary component of $A_{L}$ (long), which was $25 \mu$ s in length and was intended to discriminate against $\mu$-decay electrons. The other components of $A_{L}$ were $A_{6}(a \cdot b)$, Inserted here because it was not a component of $A_{H}$, $A_{7}$ (the second layer of top scintillators), and $C_{H} \cdot A_{S}$, which was included to catch events missed by an inefficiency in $A_{H}$. The length of this gate meant that its rate had to be kept fairly low in order to keep the dead time at an 
acceptable level. Typical rates for $A_{L}$ were $5 \mathrm{~K} / \mathrm{s}$ giving a dead tine frow that one source of $12 \%$.

The drift chambers, because of the 10-cm wire spacing, had a tiae Jitter of about I $4 \mathrm{~g}$, making it Impossible to put thea into the fast electronics in a way corresponding to the scintillator antis. Sigtals from all che wires in a single chamber were mixed together to give a single signal from each chamber; the four signals were wixed in the tratler. This ylelded a gate which went into the CAMAC gated colncicience buffer used for the event latches, and any event (or clock) with that latch set was not analyzed but simply thrown out.

The event definitions started with a $C \cdot V$ colncidence, to incorporate the voter. A computer busy signal was also in this colnctdence as an anti; this signal was set by the event latch and terminated by the computer when processing of the event was complete. A random nolse generator was used as a clock to measure the live time; for maxlmum accuracy these clocks needed to look as much like events as possible. They were, therefore, mixed into the gates for $C \cdot V, C_{L}$, and $C_{J} \cdot \bar{C}_{R}$, went through the same event channels as normal events, and were separated only by a latch in the CAMAC reglster (see below).

The following is a list of all the event types that were used, wth a brief description of each:

$$
\begin{aligned}
& A_{1} \cdot A_{6}: \quad \text { consisted of } A_{1} \cdot A_{6} \cdot C \cdot V \text {; throughgoing cosmic rays, } \\
& \text { used as an energy calibration. } \\
& \text { DTM: } \\
& \text { conststed of }\left(C_{J} \cdot \overline{C_{K}}\right)\left(\overline{A_{L} \text { or } A_{S} \text { delayed }}\right) \text { these were } \\
& \text { high-energy charged events with no beam-associated } \\
& \text { component, but with the same live time as true }
\end{aligned}
$$


neutrals (the reason for including the delayed ant1); used to check sibtraction. Rate was adjueted by changing width of window.

$C \cdot \overline{A_{L}}: \quad$ consisted of $C \cdot V \cdot \overline{A_{L}} ;$ low-energy neutral events, separated from higher-energy events because of high rate.

$C \cdot C_{L} \cdot \overline{A_{L}}:$ consisted of $c \cdot v \cdot C_{L} \cdot \overline{A_{L}}$; best neutrals, these were the neutrino candidates.

$C \cdot C_{L} \cdot \overline{A_{S}}:$ consisted of $C \cdot V \cdot C_{i} \cdot \overline{A_{S}}$, later (after rua 933) of $c \cdot V \cdot \overline{A_{S}} ;$ mostly muon decays, used for energy calibration and checining effect of overshoot. (The overshoot problem is discussed below, under "Checks and calibrations.")

$\mu$ Decay: consisted of $\quad\left[\left(C \cdot V \cdot \overline{C_{M}} \cdot A_{H}\right)\right.$ delayed $2 \mu s)\} \cdot C \cdot V \cdot \overline{A_{S}} ;$ upper level was to avo1d overshoot problems, as was delay; used to set gala of counter since energy is the same as for neutziao events. Changed to $\left[\left(A_{7} \cdot A_{H}\right)\right.$ delayed $10 \mu \mathrm{s}] \cdot \mathrm{C} \cdot \mathrm{V} \cdot \overline{\mathrm{A}}_{\mathrm{S}} \cdot \overline{\mathrm{A}}_{\mathrm{L}}(9 \mu \mathrm{s})$ to give triggers throughout volume of counter and to reduce accidentals.

Each event gate went through a prescaler and into a common event mixer and fanout; the normal prescalings were as follows:

$$
\begin{array}{ll}
A_{1} \cdot A_{G} & \times 100 \\
\text { DTM } & \times 1(\text { to keep its live time the same as the }
\end{array}
$$


neutrels)

$$
\begin{array}{ll}
C \cdot \overline{A_{L}} & \times 100 \\
C \cdot C_{L} \cdot \overline{A_{L}} & \times 1 \\
C \cdot C_{L} \cdot \overline{A_{S}} & \times 100 \\
\mu \text { Decay } & \times 10 \text { (old definition) } \\
& \times I \text { (new definition) }
\end{array}
$$

The sole purpuse of the prescaling was to keep the event rate reasonable; with this set, the total event rate was about $6 / 11$ ve secoud. A separate latch for each event type went to a CAMAC gated colncidence register; this register also recelved iatches for beam on/off, biomed target In, and clock (this is how the clocks were distinguished from true events). The register was gated by the event mixer/fanout and read in for each event and clock. Table VII shows the CAMAC register word, with the definition of each bit. 
TABLE VII

CAMAC COINCIDENCE REGTSTER WORD

$\begin{array}{cl}\text { BIt } & \text { Definition (B1t }=1 \text { 1f true) } \\ 0 & \text { Beam Out } \\ 1 & \text { Bio In } \\ 3 & \text { Clock } \\ 4 & \text { A }_{1} \cdot A_{6} \\ 5 & \text { DTM } \\ 6 & \text { C } \cdot \mathrm{C}_{\mathrm{L}} \cdot \overline{A_{L}} \\ 7 & \text { C } \cdot \overline{A_{L}} \\ 8 & \text { C } \cdot\left(C_{L}\right) \cdot \overline{A_{S}} \\ 9 & \text { Mu Decay } \\ 18-15 & \text { Beam In } \\ 16 & \text { not used } \\ 17 & \text { Event } \\ 15 & \text { not used }\end{array}$


CHAPTER 6

DATA ACQUISITION

\section{A. DaTA ACQUISITION}

The on-line data acquisition computer was a Data General Supernova.65 Figure 34 is a simplifled flow chart of the data-acquisition program. The event mixer and fanout sent a signal to the enabling gate of the $\mathrm{C} 212$ gated colncidence register when an event was recelved; this then generated a LAM in the CAMAC crate. Upon recelpt of the LAM, the compster went into the event hariding routine outlined in the figure. If the "clock" bit was set, the event and Its ADC readings were accumulated; all non-clock events were written on tape with no further selection. The only exception to this were events where the drift chamber bit was set; all such events and clocks were thrown out without ever being stored.

Beam gates were produced from an analog signal produced by a current monitor just upstream of the $A 6$ beam stop. The length of this signal was 500 us foz a $6 \%$ dury factor, and $625 \mu \mathrm{s}$ for a $7.5 \%$ duty factor; the repetition rate was $120 \mathrm{~Hz}$, or 8.3 ms between beam spills. Two gates ware generated from this pulse: a 500- or 625-is prompt gate, and a 3- or 3.75-ms delayed pulse, which was used zo measure the cosmic-ray background. The time structure of these puises is shown in

65. Data General Corporation, 15 Turapike Rä., Westboro, KA 01581 . 

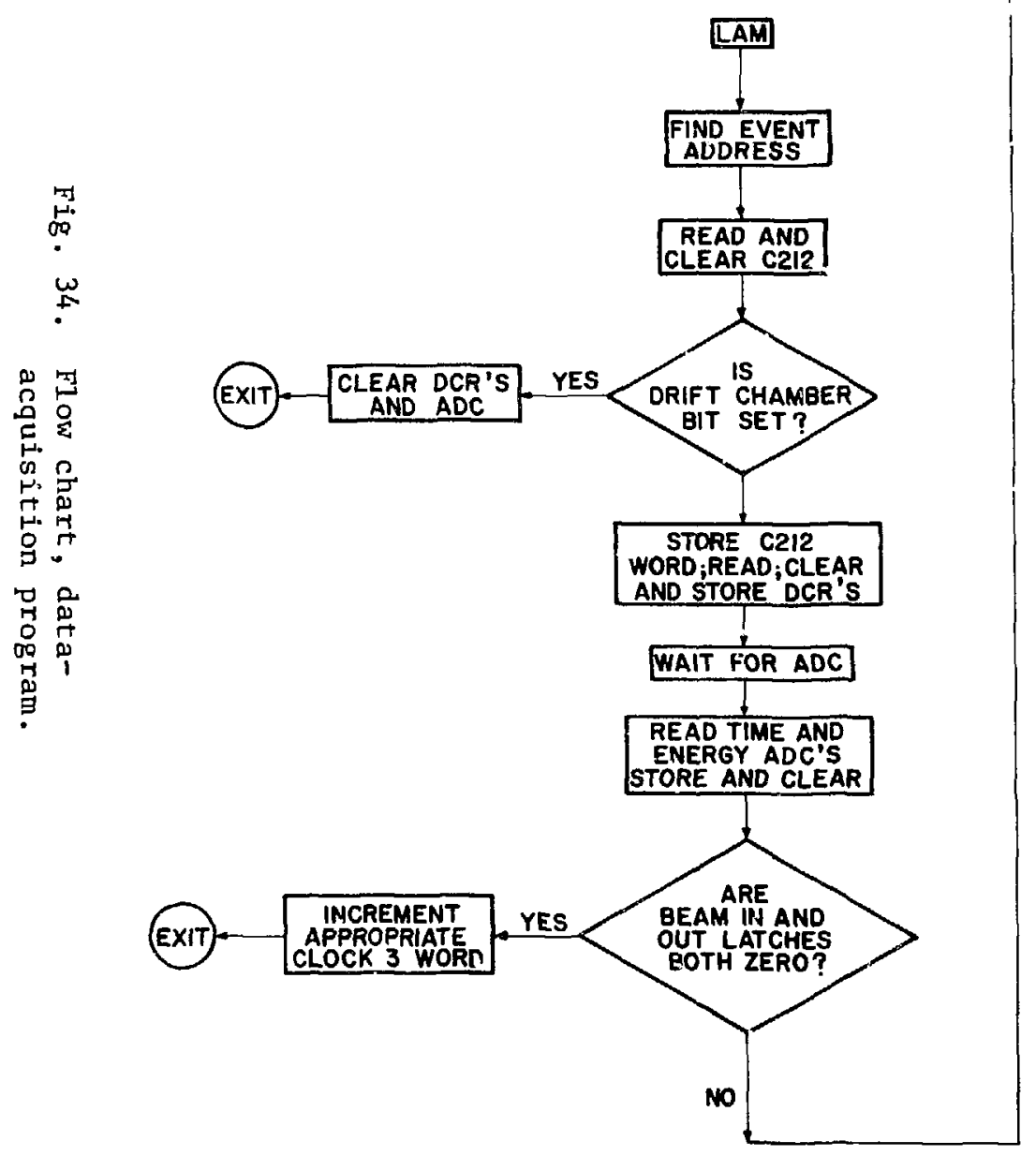

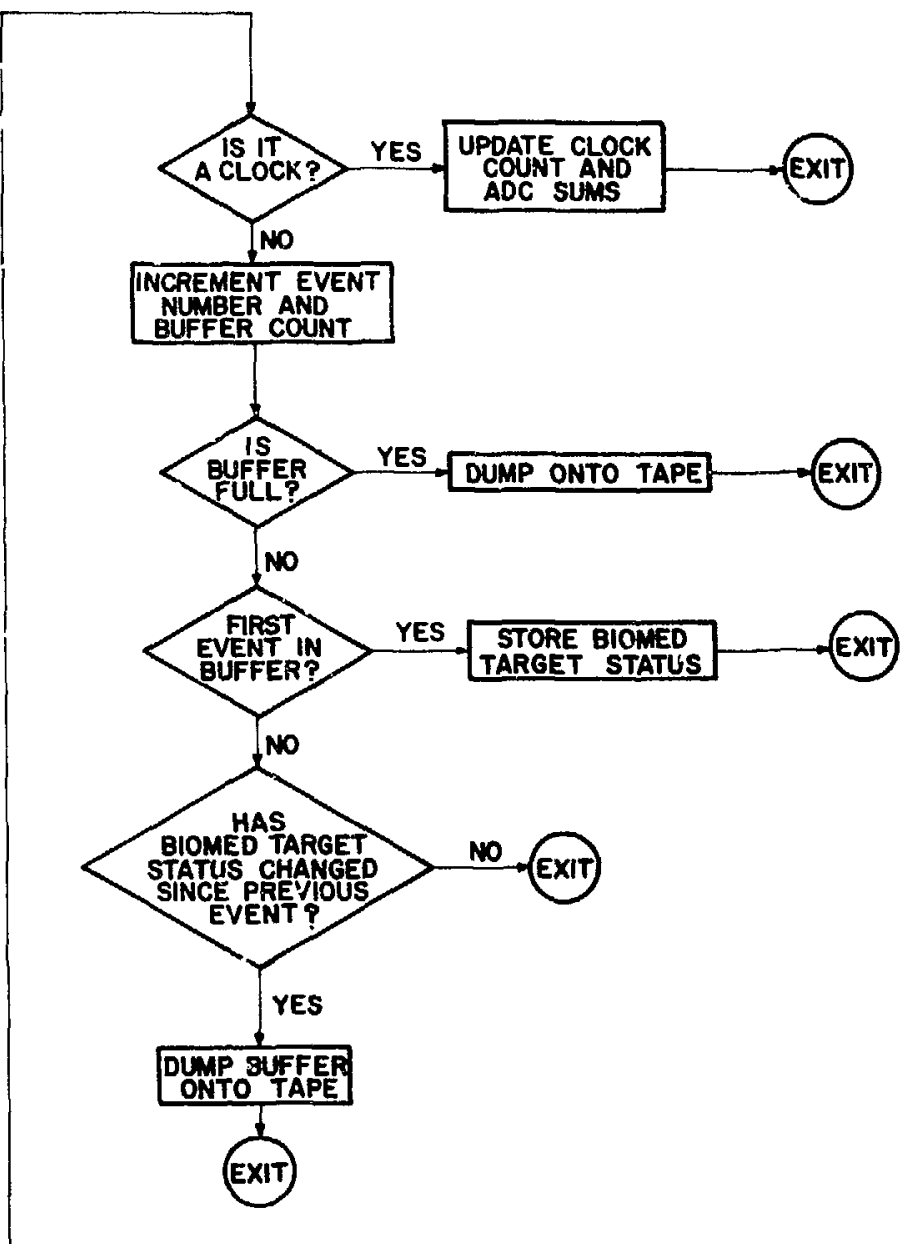

$\frac{1}{1}$ 

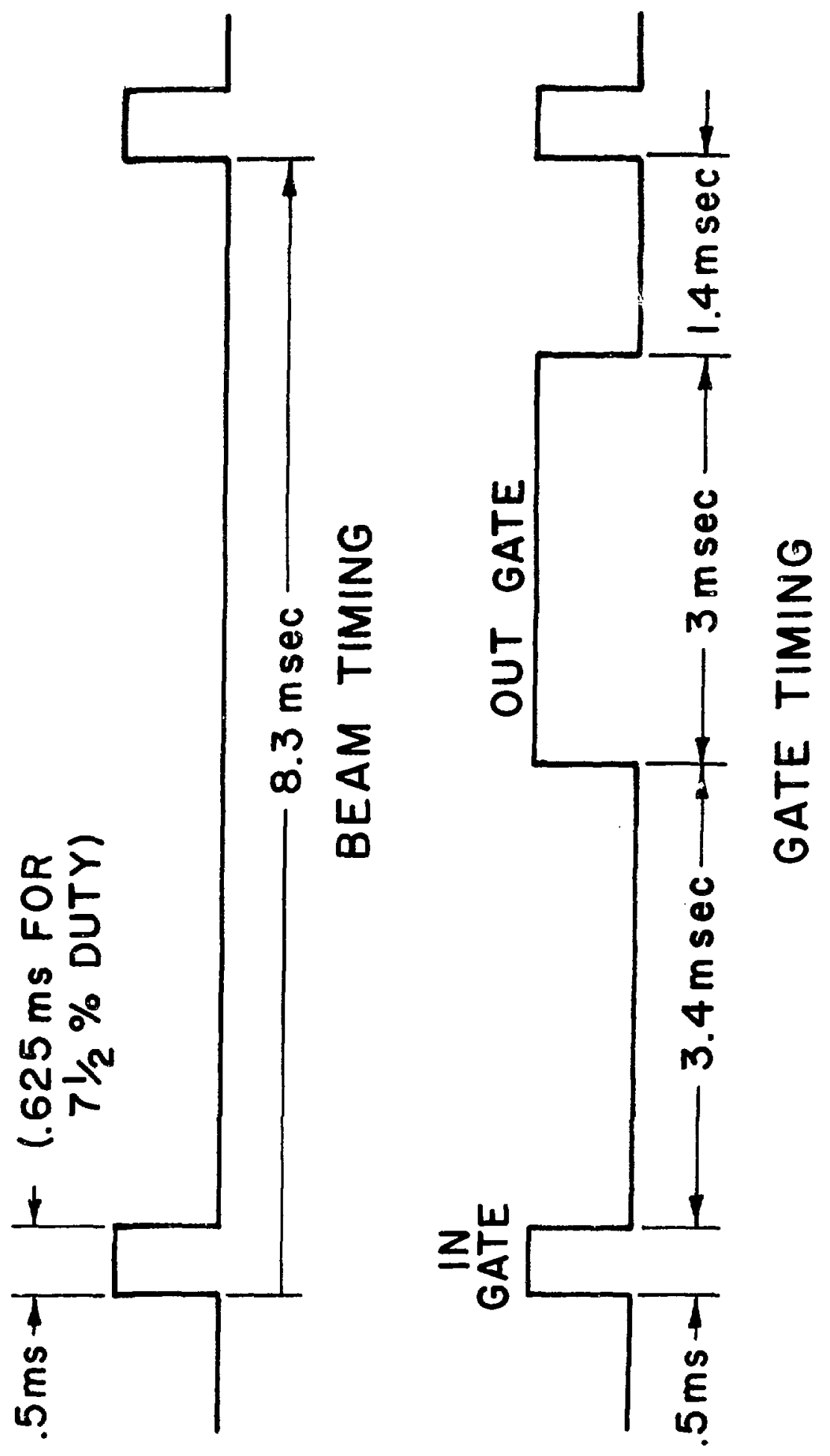

F1g. 35. Beam and gate timing. 
Fig. 35, along with the time structure of the actual beam opill. These "beam In" and "beam out" gates each had a separate latch in the C212, as shown In Table VII.

In order to make a subtraction of the cosmic-ray background as measured during the out gate, it was necessary to make an accurate measurement of the live time during each gate. This was done by having a random clock "or"ed Into the event logic, as described in the previous section. At the end of each run, a ratio would ba made of the clocks in the in and out gates, for each blomed target condition, and this number would be the factor by which the "out" events would be renormalized. The live time for the clocks should have been the same as the live time for the events, since the clocks were random in time, had the same sources of dead time as the events, and were not prescaled. The subtraction was checked frequently whenever the beam was off for several days; the results were not stacistically different from $z \in$ ro.

Table VIII shows a list of Inputs to the five hex CAMAC scalers. Two of these units were gaied "beam 1n", two were gated "beam out", and the fifth was ungated, as indicated in the table. "Live event" scaled the event fanfnout, vetoed by the live clock. " $\mathrm{C}_{\mathrm{H}}$ " was the high-level (100-MeV) Cerenkov total rate. "LIve clock" was a coincidence between the event faninout and the cilock. "Clock" scaled the clock directly. "A6 $\cdot \mathrm{C}_{\mathrm{H}}$ " gave a large signal in the Cerenkov counter in coincidence with a signal In the bottom scincillator; "A ${ }_{6} \cdot \mathrm{C}_{H} \cdot \bar{A}_{H}$ " was this signal witn nothing in the side or top scintillators. The ratio of these two measured the ant1 Inefficlency. " $C_{H} \cdot A_{7}$ " was a colncidence between a large signal in the Cerenkov counter and the second layer of top scint1llators. " $\mathrm{C}_{\mathrm{H}} \cdot \overline{\mathrm{A}}_{\mathrm{L}}$ " was a large signa1 in the Cerenkov counter 
with nothing in any of the antis. " $P_{6}$ " was an integrated pulse train from the current monitor just upstream of the beam stop; the number of pulses in each sp111 was proportional to the amount of beam in the spill. "C $C_{H}$ - DC" was a colncidence betweer a large signal in the Cerenkov counter and the drift chambers. "1.1 KHz" was a regular (nonrandom) clock whose function was to measure the total elapsed time. "Drift chamber" scaled the chambers directly. "A," scaled the second layer of top scintillators. "Voter" scaled the output of the voter colncidence unit. "A ${ }_{S}$ " was the low-threshold scintillator output, and "A ${ }_{H}$ " the high threshold. " $\mathrm{C} \cdot \mathrm{V}$ " was the colncidence between the 3-MeV Cerenkov discriminator and the voter. "Mu stop" was the coincidence $A_{7} \cdot A_{H}$ used to define a stopping muon for the $\mu$-decay event trigger. "Beam gates In" scaled the number of beam spills with beam. "T zero gates" scaled the master timer of the accelerator; this normally ran at $120 \mathrm{~Hz}$.

The scalers were bonitored on IIne by the computer, and certain ratios of the scalers were checked periodically to see if they fell within certain limits. If not, a warning containing the numbers of the scalers, the value of the ratio, and the time was printed out. Table IX 18 a list of these warnings. The first measured live events per second for beam In; this could have been wrong if the dead time were $h_{1}$ gh, or if something were wrong with the antis. The second measured the anti Inefficiency. The third measured the rate in the scintillators. The fourth measured the drift chamber rate. The fifth was the dead time, excluding dead time due to the drift chambers. The sixth, $1.1 \mathrm{KHz} / \mathrm{T}$ zero gates, would have been off if $\mathrm{T}$ zero were not at $120 \mathrm{~Hz}$, as sometimes occurred when the accelerator was having problems. The 
TABLE VIII

\section{CAMAC SCALERS}

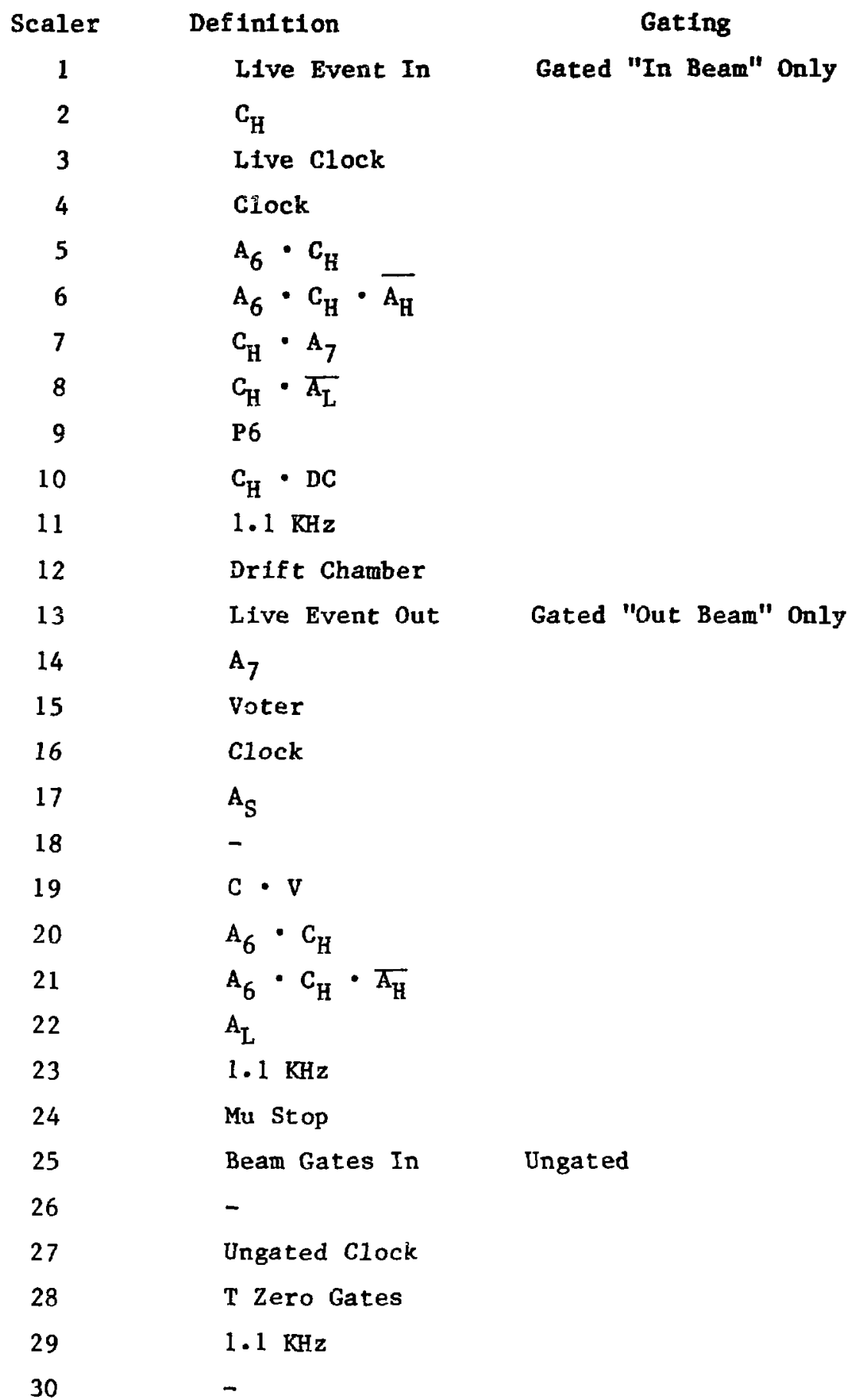


seventh was the $C \cdot V$ rate. The eighth was $A_{7}$, the second layer of top scintillators. The ninth was the event rate for beam out. The tenth measured the clock ratio, beam in/beam our; this could vary if the clock were not running at the same rate during each gate, which sometimes occurred via plckup from the line voltage.

Figure 36 is a copy of the run sheets that were filled out at the end of each run. This Involved checking the rates of all the scalers, the rates of each event type, the cosmic-ray neutrals rate, and the beam-associated rates oi the neutral events. This enabled us to keep close track of the condition of the entire experiment, and to fix things shortly after they went wrong. These sheets were not used for any part

TABLE IX

SCALER WARNINGS

$\begin{array}{ccll}\text { Number } & \text { Scalers } & \text { Minimum } & \text { Maximum } \\ 1 & 1,11 & 0.002 & 0.02 \\ 2 & 21,20 & 0 & 0.0005 \\ 3 & 22,23 & 3 & 9 \\ 4 & 12,11 & 3 & 7 \\ 5 & 3,4 & 0.6 & 1 \\ 6 & 29,28 & 9.1 & 9.6 \\ 7 & 19,23 & 0.5 & 0.7 \\ 8 & 14,23 & 0.5 & 1 \\ 9 & 13,23 & 0.002 & 0.02 \\ 10 & 4,16 & 0.175 & 0.185\end{array}$




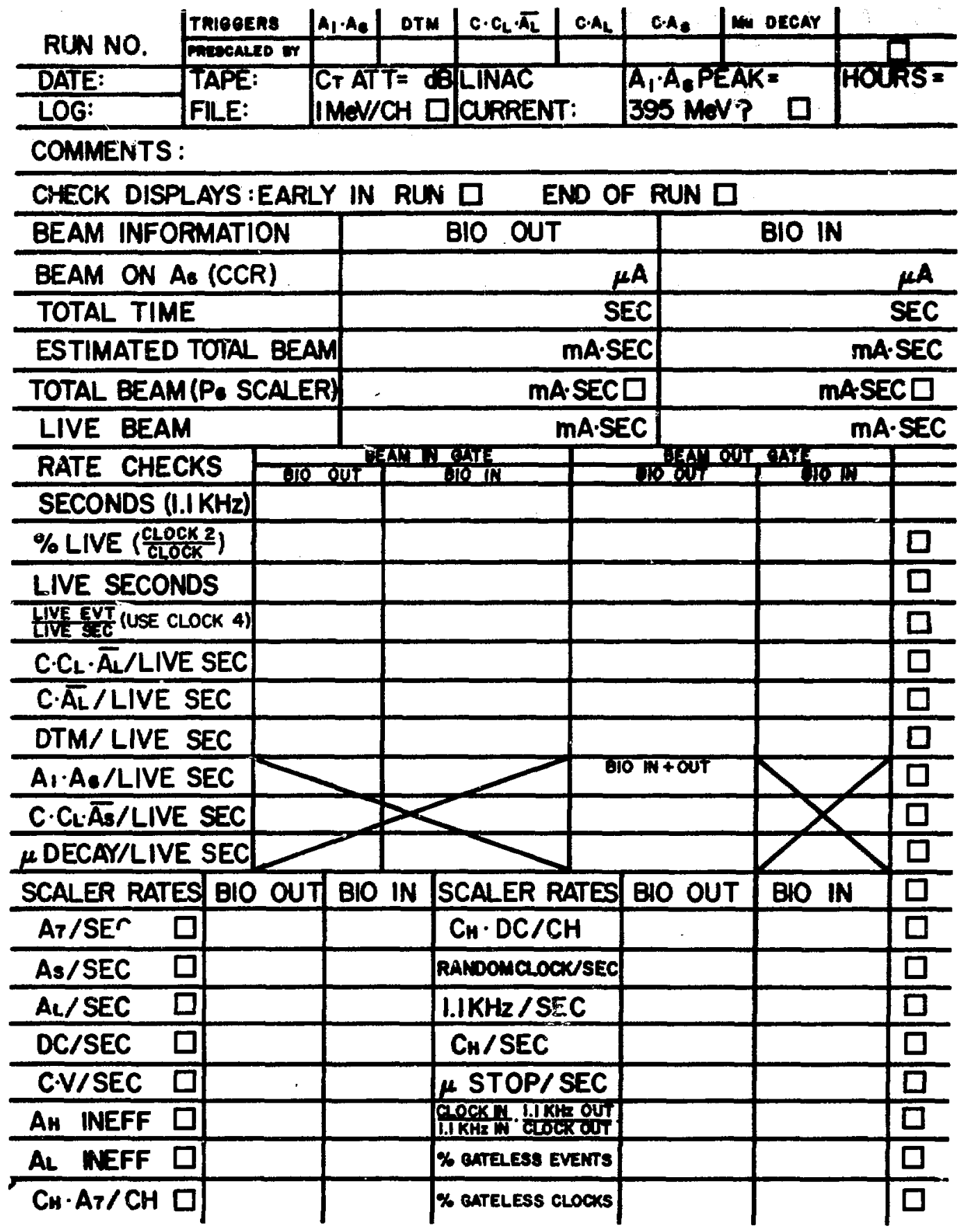

Fig. 36. Run sheet. 
PAGE 2 - RUN NO.

SPECTRA

\begin{tabular}{l|l|l|l|l}
\hline \hline \multicolumn{2}{l|}{ COSMIC NEUTRAL: BEAM OUT ONLY. } \\
\hline & BIO OUT & BIO IN & $\Sigma B 1 O$ IN + OUT & \\
\hline LIVE LADAYS (BEAM OUT) & & & & \\
\hline NEUTRALS $\Sigma(20,60) /$ LNVE & & & \pm & $\square$ \\
\hline NEUTRALS $\Sigma(30,60) /$ LAOEY & & & \pm & $\square$ \\
\hline
\end{tabular}

BEAM ASSOCIATED RATES

\begin{tabular}{|c|c|c|c|c|c|c|}
\hline & & IN GATE & $\begin{array}{l}\text { OUT OATE } \\
\text { (MUNOANM) }\end{array}$ & $\Delta$ & AOt $(\%)$ & $\Delta /$ Live $(\mathrm{mc})$ \\
\hline \multirow{2}{*}{5} & DTM & & & \pm & \pm & 口湈采: \\
\hline & NEUT. $\Sigma(30,60)$ & & & \pm & \pm & $\square$ \\
\hline \multirow{2}{*}{ 음 } & NEUT. $\Sigma(10,30)$ & & & \pm & \pm & $\square$ \\
\hline & C. $\overline{A L} \quad \Sigma(5,10)$ & & & \pm & \pm & $\square$ \\
\hline \multirow[b]{2}{*}{$\underline{z}$} & $\overline{D T M}$ & & & \pm & \pm & 口蛅: \\
\hline & NEUT. $\Sigma(30,60)$ & & & \pm & \pm & $\square$ \\
\hline \multirow{2}{*}{$\frac{\text { 음 }}{\text { D }}$} & NEUT. $\quad \Sigma(10,30)$ & & & \pm & \pm & $\square$ \\
\hline & $C \cdot \overline{A L} \quad \Sigma(5,10)$ & & & \pm & \pm & $\square$ \\
\hline
\end{tabular}

ACCUMULATED RATES: GOOD RUNS STARTING WITH NO.

\begin{tabular}{|c|c|c|c|c|c|}
\hline & & 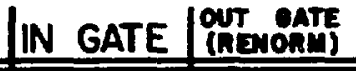 & $\Delta$ & 这 $(\%)$ & $\Delta$ LLIVE $(m c)$ \\
\hline \multirow{2}{*}{5} & DTM & & \pm & \pm & HE: \\
\hline & NEUT. $\quad \Sigma(30,60)$ & & \pm & \pm & $\square$ \\
\hline \multirow{2}{*}{ 음 } & NEUT $\Sigma(10,30)$ & & \pm & \pm & $\square$ \\
\hline & $\overline{C \cdot \overline{A L}} \quad \Sigma(5,10)$ & & \pm & \pm & $\square$ \\
\hline \multirow{4}{*}{$\begin{array}{l}\text { 음 } \\
\text { 음 }\end{array}$} & $\overline{\text { DTM }}$ & & \pm & \pm & HEk \\
\hline & NEUT. $\Sigma(30,60)$ & & \pm & \pm & $\square$ \\
\hline & NEUT. $\Sigma(10,30)$ & & \pm & \pm & $\square$ \\
\hline & C. $\overline{\mathrm{AL}} \quad \Sigma(5,10)$ & & \pm & \pm & $\square$ \\
\hline
\end{tabular}

Fig. 36. Run sheet

(continued). 
of the off-IIne analysis, however; that was done directly from the original data tapes by a larger computer, and w11 be described in another chapter.

The on-IIne analysis program also put events into histograms which were stored on disk files; these disk files were written oato tape periodically, and were useful for diagnostics, trouble-shooting, and some analysis. Table $X$ shows a list of these histograns, along with the latch pattern for each histogram as read from the C212 word (see Table VII). These latch patterns were exclusive; that 18 , the given pattern, with no additions or deletions, must have matched the C212 word for an event to have been accepted into any given histogram.

\section{B. CALIBRATIONS AND CHECKS}

We had a number of ways in which to check the energy callbration of the counter -- the energy spectrum of decay electrons from cosmic-ray muons; throughgoing cosmic rays; and events from the $C \cdot C_{L} \cdot \bar{A}_{S}$ trigger. The $\mu$-decay electrons were the most pertinent check, since their energy spectrum was the same as the $\mu$-decay antineutrinos from the beam stop, and since they were the same type of particle, as opposed to muons, which did not necessarily have the same energy loss per centimeter at the same energy as electrons. This spectrum was therefore used to set the gain of the counter, by comparing it with a theoretical Monte Carlo spectrum. The overshoot from the phototubes was a particular problem for this spectrum, however, because the $\mu$-decay electron, of energy 0 to $53 \mathrm{MeV}$, followed directly after a muon which could have an energy up to $400 \mathrm{MeV}$. A $1 \%$ overshoot on the muon signal could therefore shift the electron signal $10 \%$ or more. Our original way 
TABLE $\mathrm{X}$

SPECTRUM DEFINITIONS

\section{Spectrum}

0 Neutral Beam/I Bio/O

1 Neutral Beam/O Bio/O

2 Neutral Beam/I BIo/I

3 Neutral Beam/O BIo/I

4 DCRs

$5 \quad A_{1} \cdot A_{6}$ Beam Out

$6 \mathrm{C} \cdot \mathrm{C}_{\mathrm{L}} \cdot \overline{\mathrm{A}_{\mathrm{S}}}$

$7 \mathrm{C} \cdot \overline{\mathrm{A}_{\mathrm{L}}} \mathrm{Beam} / \mathrm{I} \mathrm{B10} / 0$

$8 \mathrm{C} \cdot \overline{\mathrm{A}_{\mathrm{L}}}$ Beam/O Bio/O

$9 \mathrm{C} \cdot \overline{\mathrm{A}_{\mathrm{L}}} \mathrm{Beam} / \mathrm{I} \mathrm{B1o} / \mathrm{I}$

$10 \mathrm{C} \cdot \overline{\mathrm{A}_{\mathrm{L}}} \mathrm{Beam} / 0 \mathrm{BLo} / \mathrm{I}$

11 Mu Decay ADC 0

12 DTM Beam/I Bio/O

13 DTM Beam/O Bio/O

$14 \mathrm{DTM} \mathrm{Beam} / \mathrm{I} \mathrm{Bio} / \mathrm{I}$

15 DTM Beam/O Bio/I

16 Time Beam/I Bio/0

17 Time Bean/o Bio/0

18 T1me Beam/I BLo/I

19 Time Beam/O Bio/I

\section{Latches}

1340,1360

341,361

1342,1362

343,363

7777 (dummy)

11,13

201,203

1300

301

1302

303

$401,403,601,603$

$1020,1030,1360$

$21,31,361$

$1022,1032,1362$

$23,33,363$

7776 (dummy)

7776 "

7776

7776 "

of avoiding this problem in the $\mu$-decay trigger was to put an upper limit of about $30 \mathrm{MeV}$ on the muon signal, thus reducing by a factor of 
10 or more the maximum sixe of the overshoot. Th18, unfortunately, had the effect of restricting the decay electrons to a linited portion of the counter, and they were therefore not as simtlar to the neutrino events as they could hare been, since the neutrino everts will occur uniformly throughout the counter. We therefore decided to redefine the stopping muon trigger to consist just of the antis $A_{7} \cdot A_{H}$ (the coincidence was to reduce noige) with no constraints on the Cerenkov signal at all. We then looked for a second signal delayed by $10 \mu s$, which was long enough to eliminate the overshoot problem. We included a 9 us ant1 to ensure that the electron we were looking at came from the original muon and not from any other muon which may have stopped in the counter during the delay time. Figure 37 shows the measured $\mu$-decay spectrum with this final definition, and the Monte Carlo calculation of the spectrum. The energy scale of the Monte Carlo spectrum was adjusted to make the peaks coincide.

The throughgoing cosmlc rays were defined by a coincidence of the top and botton antis, $A_{1} \cdot A_{6} \cdot$ The expected position of this peak was we:ll-defined by the size of the counter, so it could be used on line to adjust the gain of the counter, which we tried to keep at 1 MeV per channe1. The Intrinsic gain of the counter could change due to vartations in the cloudiness of the water and to fluctuations in the gains of the phototubes. The gain indicated by this trigzer could be compared to the gain Indicated by the $\mu$-decay trigger as a consistency check. 


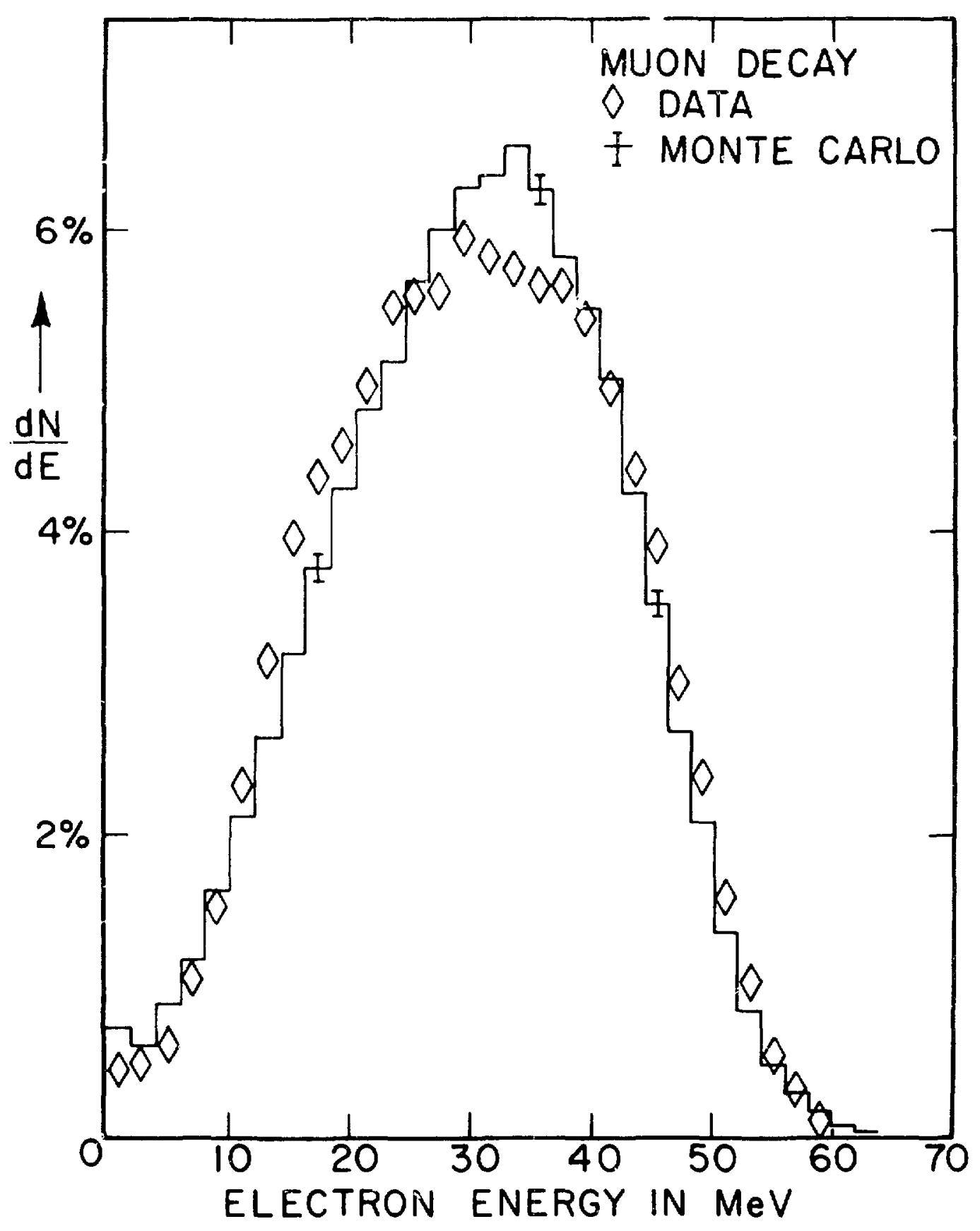

Fig. 37. Measured and calculated muon decay spectra. 
The $\mathrm{C} \cdot \mathrm{C}_{\mathrm{L}} \cdot \overline{\mathrm{A}}_{\mathrm{S}}$ trigger also conalated primarlly of mon decays; $1 \mathrm{t}$ was not too useful by itself Bince it suffered from the overshoot problem described above, but it was useful to compare it th the mon decay trigger to see the maximum effect of the overshoot.

As mentioned above, all the phototubes on both the Cerenkov counter and the scintillators were provided with LEDs to allow testing. We had computer programs which would flash each LED in succession, read in the ADCs, and print out a table of readings for all the tubes. This allowed us to check that all the tubes were functional. These tests were run dally when we were taking data. We could also tell if the gain of a tube had changed, but we did not use these readings to set the gains, as our experience was that the gains were very stable on a day-by-day basis. What we did was to set the gains of all the tubes at once, usirg throughgolng cosmic rays as a light source. This was done every few months.

The subtraction was checked run by run using the DTM trigger described above. The subtraction was also checked for an entire cycle summed together, and for the total data. The results were not significantly different from zero (but see the comments on Cycle 18, In the chapter on analysis). The subtraction for all the running was $(-0.10 \pm 0.11) \%$

\section{HISTORY OF THE EXPERIMENT}

The following chart gives a brief outline of the history and development of the experiment, noting general activities and important milestones and events. 
1970:

15 March 1971:

January 1972 :

June-July 1972:

10-31 August 1972:

5 November 1972-

15 February 1973:

1 Apr11 1973:

20 June 1973:

Summer 1973:

17 December 1973:

25 Febratary 1974:

3 March 1974:

14 March 1974:

5 May 1974-6 June 1974: Beam studies with Cerenkov counter, 16 tubes, 2- $\mu \mathrm{A}$ beam

25 October 1974:

Small prototyge Cerenkov counter butlt and tested at Saclay

LAMPF proposal submitted

Testing of sample: phototubes and prototype base at Yale

Testing of prototype Cerenkov courter at Yale, with antis

Measurement of bear-assoclated rates in prototype Cerenkioy councer at Berkeley cyclotron

Tests of prototype $C_{t}$ renkov counter in neutrino tunnel at BNL (cosmic-ray studies) Testing of epoxy castings at Yale Start of testing of scintillators for antis, at LAMPF

Cerenkov counter bullt; SEW joins experiment First test of Cerenkov counter with phototubes, at Yale

Radiation levels in LAMPF neutrino house measured, 2- $\mu \mathrm{A}$ beam

FIrst look at beam-associated events at LAMPF with prototype Cerenkov counter

Cerenkov counter installed in neutrino house

Top and bottom antis installed 
3-19 December 1974 ;

i9 December 1974:

Juiy-December 1975:

5 April 1976:

Apr11-June 1976:

18-20 June 1975:

June-kovember 1976:

July-Ocyober 1976:

November 1976-

March 1977:

1 June 1977:

13 June 1977:

13 July 1977:
Beam studies, all tubes mounted, 10- to 13-uA beam, shielding plug ralsed on 16 December Start of Great Shutdown of LAMPF accelerator Scintilletor stands set up, scintillatora nounted, tubes put on and tested, stands moved in place

End of Great Shutdown; Cycle 1, 10-uA beam Measurements of beam assoclared background at $3.25 \mathrm{~m}$ and $4 \mathrm{~m}$ of shielding Fifth meter of shielding added Measurements of beam assoclated background at 5 m of shielding Measurements of effect of varying amounts of lead on cosmic rays Installation of last $1.3 \mathrm{~m}$ of shielding; experiment disassembled, moved back, and reassembled

$A_{7}$, second layer of top antis, installed Drift chambers Installed; system complete Cycle 16, 225- $\mu$ A beam, start of data-taking

Detalls of beam-associated running conditions are in the final chapter, with the results.

October-November 1977: Setup disassembled, counter repalred, reassembled 
Apr11-May 1978:

Setup disassembled, counter repaired, made ready for $D_{2} O$ fill; filled and reassembled

June-September 1978: $\quad D_{2}{ }^{O}$ running

8 November 1978: Resume water running

7 May i979:

End water running 
CHAPTER 7

ANALYSIS

The analysis of this experiment consisted primarily of sorting events into histograms based on event type, blomedtcal target condition, and beam condition; rencrmalizing the "outside beam" spectrum to correspond to the same amount of live time as the "inside beam" spectrum; subtraciing to find a beam assoclated spectrum; and fitting this spectrum to a calculated neutrino spectrum to find a value for the branching ratio. A few cuts were made on the data before putting them into histograms; these will be described below.

The format of each event as written on tape is shown in Table XI. Events were written on tape in sets of 200; each contained 200 events with 8 words per event, followed by clocks and clock sums, and scalers, accumulated since the last tape dump. When rereading these original tapes, a run sheet (see Fig. 36) was calculated. This allowed us to check rates and subtractions, Just as the on-line hand calculated ones did. There were also options for up to 5 scatter plots and 20 histograms to be generated. Variables could be defined from the Information on the tape; these variables could then be plotted. Cuts could be defined by placing limits on the variables; a maximum of 20 cuts could be defined at any one time. Each histogram or scatter plot could have up to 10 cuts applied to it. The program would also 
TABLE XI

TAPE FORMAT

Word

1

2

3-4

5-8

\section{Contents}

TDC reading

C212 word (see Table VII), bits 0-15

24 bits, discriminators on supertubes (DCRs)

8 ADC readings, two per word

ADC 1,2: summed signal, 1 and 5

MeV/channe1

ADC 3-8: 6 side signals

calculate subtracted spectra for the neutral events, separated according to whether the blomed target was in or out.

As mentioned above, we took a mixture of events while running, in order to be able to check gains and subtrastions on 1ine. This was necessary hut resulted in a large number of events on tape which were extraneous as far as calculating a branching ratio was concerned. The neutral events $\left(C \cdot C_{L} \cdot \bar{A}_{L}\right)$ were only about $4 \%$ of the total. We declded, therefore, to edit the original tapes onto a smaller set of tapes. These edited tapes contained all the $c \cdot \mathrm{C}_{\mathrm{L}} \cdot \overline{\mathrm{A}_{\mathrm{L}}}$ events with energies above $15 \mathrm{MeV}$, and one in five of the $\mathrm{C} \cdot \mathrm{C}_{\mathrm{L}} \cdot \overline{\mathrm{A}}_{\mathrm{S}}$ and muon decay events, for a total of about $5 \%$ of the original events. Scalers and clocks were witten as sums over entire runs. We separated the edited tapes into run cycles (so each tape contained data from only one cycle); this gave us just 8 tapes to deal with insicead of the original 61. 
When replaying the edited tapes, it was possible to make histograms and scatter plots as before. The run sheet was also calculated, vising the data on the entire tape. A subtracted spectrum was calculated, wtth the normalization of the "outside beam" spectrum being doce run by run. Information needed to calculate a branching ratio was written to disk file for use by another program. This information included the following: a title; subtracted spectra, with errors; raw (unrenormalized) spectra; live beam; binning Information; average renurmalization factors. Except for the binning information, everything was divided according to the blomed target condition.

We made several cuts on the data before calculating a branching ratio. As has been mentioned frequently, the data were separated according to whether the biomedical target was in or out. Because of the background problems with that target, we used only data taken when It was out in our calculation of a branching ratio. This had the effect of cutting out about half our total data. Another cut we made was to require at least 3 of the 24 DCR channels to have fired. The purpose of this was to reduce contributions to the neutrals from tube noise. The threshold of the DCRs was set so that approxtmately half of them vould fire for a typical muon-decay event. Scatter plots of DCRs vs energy for neutrals showed a tail of events with 0 or 1 DCR firing which extended above $50 \mathrm{MeV}$. No such tail was observed in the muon-decay events. Figure 38 shows contours from typlcal scatter plots. The effect of this DCR cut was checked on the muon decay events and found to be negligible above $20 \mathrm{MeV}$. It reduced the neutrals rate for the run 11lustrated in FIg. 38 from 475/LAday to 365/LAday. The last cut we made was on the live time. This was not known for each event, but could 


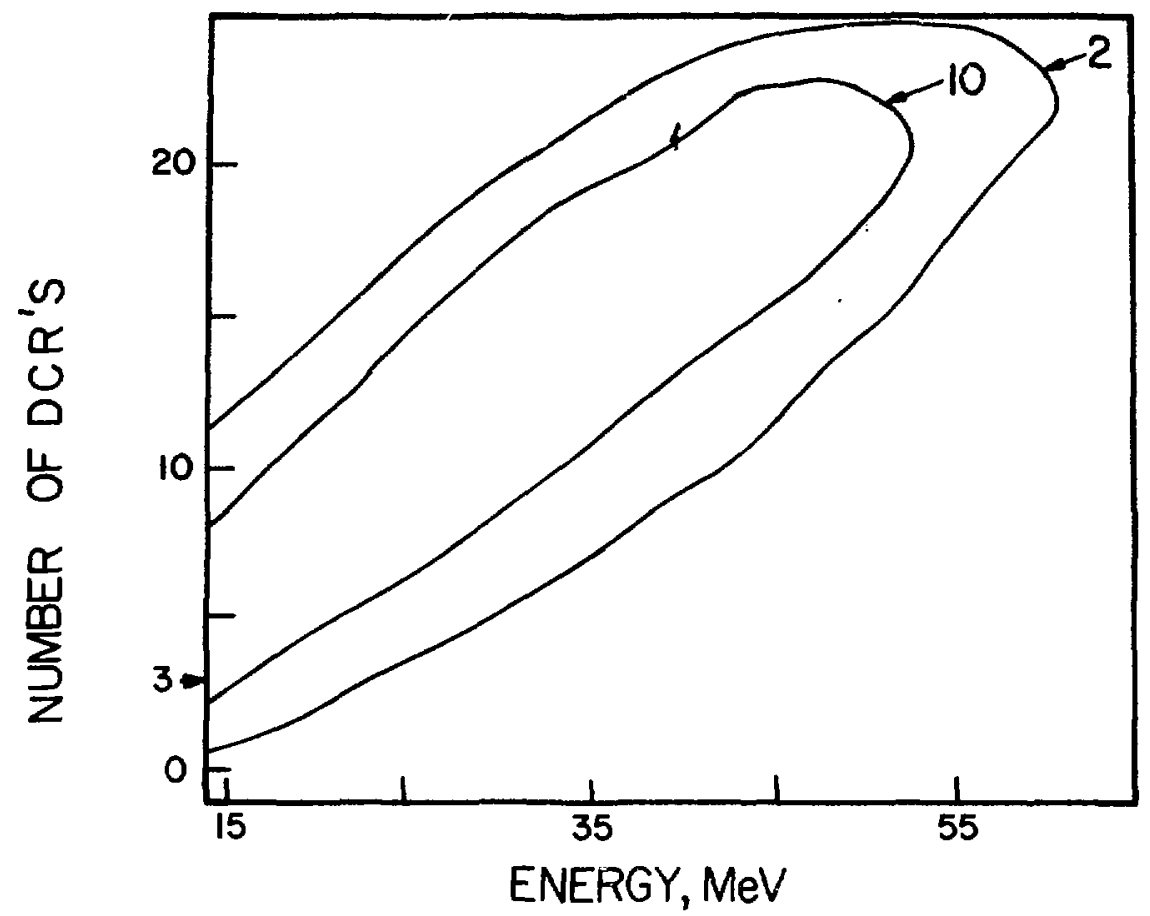

MU DECAYS

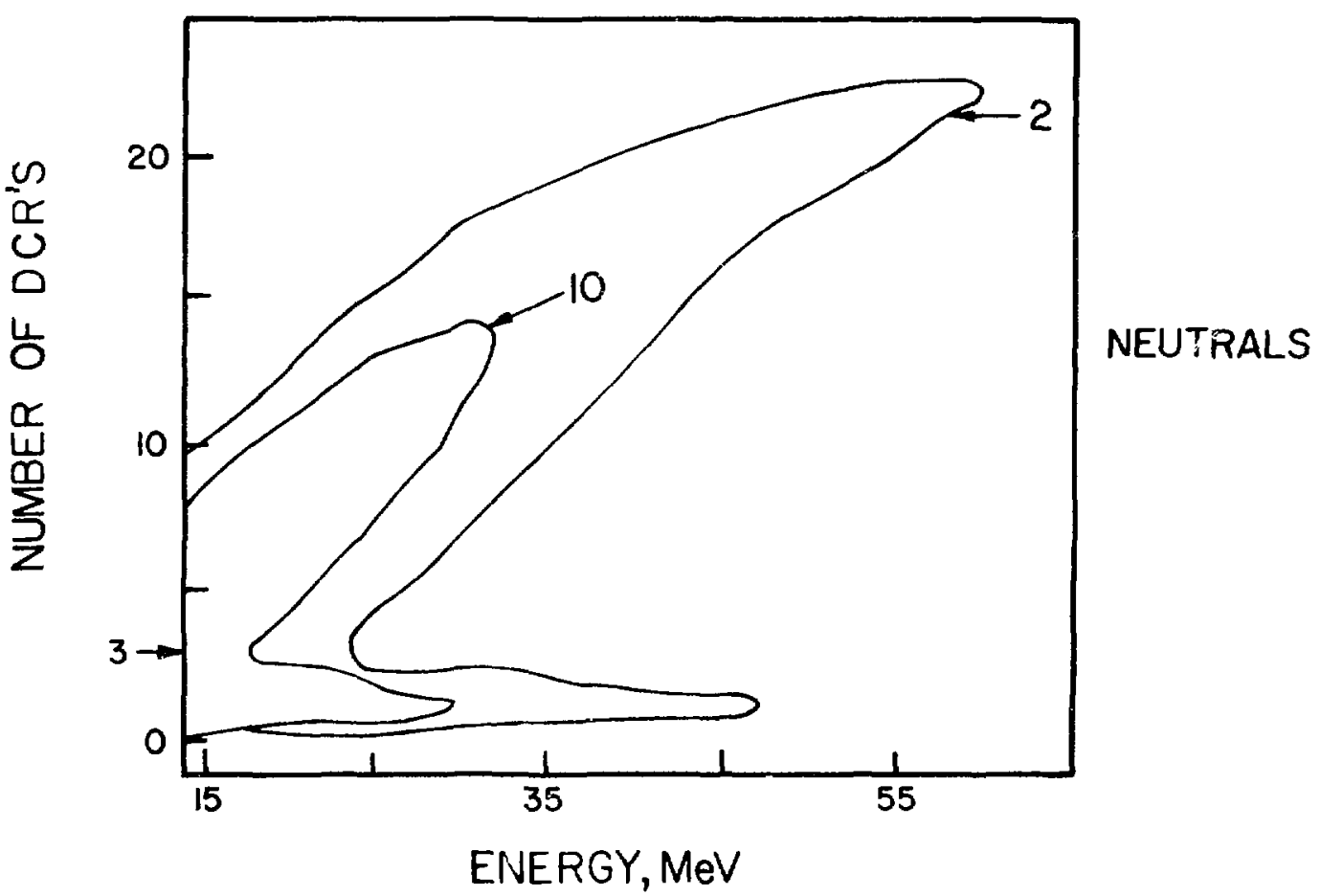

Fig. 38. Contour plots, DCRs

vs energy for muon

decays and neutrals. 
be calculated for each 200-event record from the scalers and clocks. Normilly, the live time was about $75 \%$. The radioactive gas, described In the sections on the drift chambers and the neut-tno house, reduced it on occasion to as low as $20 \%$. We worried that our subtraction schese might not work at that level, and decided to make a cut at 60\%. Since we could only make this cut at the end of a record, 1t was made during the editing process. Records where the live time was less than the cutoff were simply 1gnored. This cut removed less than $10 \%$ of our data. There was a problem during Cycle 18 (November - December 1977) whtch was solved by making a cut on the data. The dead-time monttor subtraction was not zero for that cycle; it was $(1.85 \pm 0.27) \%$. This was Investigated and found to be due to events coming between the beam gates. These events were called "beam $1 n$ " because there was no "In" latch in the CAMAC gated coincidence register at that time; events were defined as being "In" If the "out" latch was not set. We added an "In" latch for subsequent running. A cut was made on the time of these events during the beam -- they appeared to be abnormally early, making such a cut unambiguous. With this cut, the subtraction was $(-0.44 \pm$ $0.30) \%$, consistent with zero.

The program which calculated the branching ratio had an expected spectrum per unit of beam, in bins which corresponded to one ADC channel each. The spectrum shape was that generated by the Monte Carlo program mentioned in the chapter on data acquisition, and was different for $\mathrm{H}_{2} \mathrm{O}$ and $\mathrm{D}_{2} \mathrm{O}$, as shown in $\mathrm{Fig}$. 39. This spectrum was then normalized to the actual amount of beam. The data, and the expected spectrum, were then added into bins several channels wide, to 1screase the statistics in each bin. Five channe:ls per bin was a typical number, although it was 


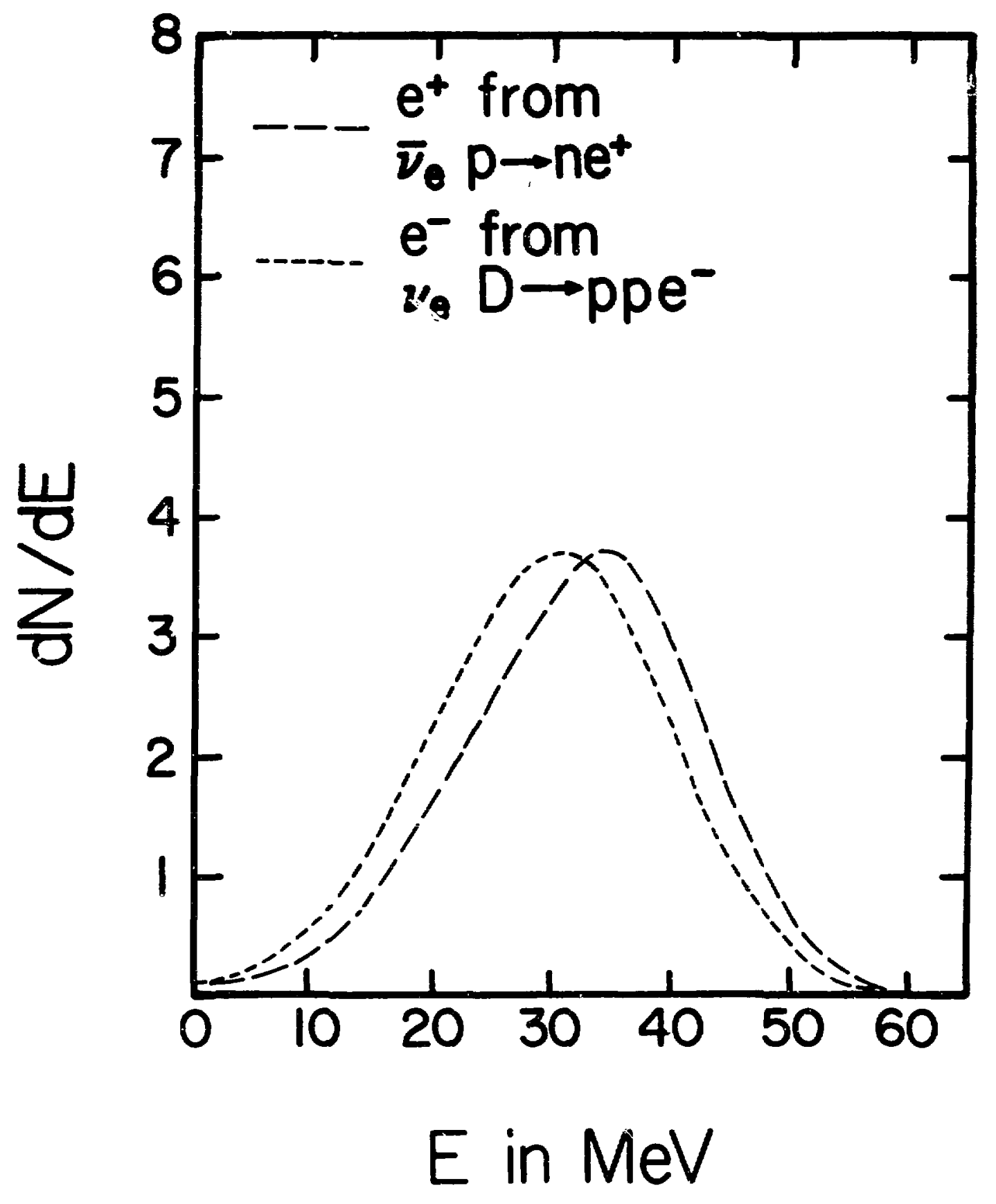

Fig. 39. Calculated spectra,
$e^{+}$from $\bar{v}_{e p} \rightarrow n e^{+}, e^{=}$ from $\nu_{\mathrm{e}} \mathrm{d} \rightarrow \mathrm{ppe}^{-}$. 
possible to choose others. A ratio was then ade, bIn by bin, of the observed signal to the expected 8 ignal $\left(S_{1}\right)$ with Its erros $\left(\sigma_{1}\right)$. A welghted average was then performed over several bins, to find a value for the branching ratio:

$$
R_{A V}=\frac{-\left(S_{1} / \sigma_{1}{ }^{2}\right)}{\sum\left(1 / \sigma_{1}{ }^{2}\right)},
$$

with the error

$$
\sigma_{R}=\frac{1}{\sqrt{\Sigma\left(1 / \sigma_{i}{ }^{2}\right)}} \text {. }
$$

The bins were selected to cover the range of substantial expected rate without getting into known low energy beam associated backgrounds. This was done for both the water and the heavy water data. The water number, $R_{H}$, was then the branching ratio for $\mu^{+}$decay to $\bar{v}_{e}$, normalized to the calculated flux. The heavy water number, $R_{D}$, was the ratio of the observed neutrino event rate to the calculated event rate. We then normalized $R_{H}$ by $R_{D}$ to cancel out any errors which may have been made in the calculation of $f$ lux or acceptance. Therefore,

$$
R=\frac{\mathbf{R}_{H}}{\mathbf{R}_{\mathrm{D}}} \text {, }
$$

with the error on $R$ calculated in quadrature from the errors on $R_{H}$ and $R_{D} \cdot$ 
The number of neutrino events per coulomb is given by the following expression:

$$
\frac{N_{v}}{q_{p}} \frac{\ell^{2}}{4 \pi d^{2}} n_{p} \sigma_{v} \ell
$$

where $N_{v}$ is the number of neutrinos per incident proton; $q_{p}$ is the charge on the proton, In coulombs; $\&$ is the length of a side of the detector; $d$ is the average distance from the detector to the beam stop; $n_{p} 18$ the number of protons or deuterons per cubic centimeter in the detector (not counting the oxygen); and $\sigma_{v}$ is the neutrino interaction cross section on the proton or deuteron, as given in the theoretical section. $N_{v}$ has been measured by chen et al. 66 to be $0.0571 \pm 0.0035$ at $720 \mathrm{MeV}$. A cascade calculation gave $0.0 \% 8$ at $700 \mathrm{MeV}$ and 0.106 at $800 \mathrm{MeV}$; Interpolating to $720 \mathrm{MeV}$ ylelded 0.083 . The energy of protons arriving at the beam stop was $780 \mathrm{MeV}$ with the Blomed target out and $732 \mathrm{MeV}$ with the Blomed in. To find $N_{v}$ at these energles, we normalized to the Chen result and used the cascade calculation to give the slope. Subsitituting in the above equation, using $\ell^{3}=180 \mathrm{~cm} \times 180 \mathrm{~cm} \times 175 \mathrm{~cm}$ and $d=9.03 \mathrm{~m}$, then gives the following results for the number of events per coulomb, assuming $R=1$ :

$$
\begin{array}{ll}
\mathrm{H}_{2} \mathrm{O}, \text { bio out: } & 2.70 \\
\mathrm{H}_{2} \mathrm{O}, \text { bio in: } & 2.35 \\
\mathrm{D}_{2} \mathrm{O}, \text { bio out: } & 0.77 \\
\mathrm{D}_{2} \mathrm{O}, \text { bio in: } & 0.67
\end{array}
$$

66. H. Chen et al., Nucl. Instrum. Meth. 160, 393 (1979). 
CHAPTER 8

RESULTS AND CONCLUSIUNS

\section{A. HISTORY OF DATA TAKING}

We begin with a history of the data taking, with dates, run numbers, average beam intensity, duty factor, water type, and live beam recelved. The live beam is computed after all cuts have been made, and is separated Into biomed target out and target in. The data are divided into run cycles, determined by the operation of the accelerator. During a run cycle, the machine ran continuously except for short (12- to 24-hour) maintenance and development periods, and the average current and duty cycle remained fixed. Run cycles were separated by periods of one week to several months. The length of a cycle started at two weeks at the beginning of our running, and increased to two months by the end.

A subtracted spectrum was accumulated for an entire run cycle in the manner described in the chapter on analysis. Run cycles were combined as a weighted sum, to accomodate the different signal to noise conditions. All running was at $800-\mathrm{MeV}$ beam energy.

Run Cycle 16: July 13, 1977 to August 4, 1977. Runs 573 to 594 . $22.5 \mathrm{HA}$ average, $6 \%$ duty factor. $\mathrm{H}_{2} \mathrm{O} .68 .6$ coulombs bio out, 48.7 coulombs bio in.

Run Cycle 17: August 14, 1977 to August 31, 1977. Rins 595 to 612. 
225 uA average, 6z duty factor. $\mathrm{H}_{2} \mathrm{O} .78 .8$ coulowbs b1o out, 40.6 coulombs b1o in.

Run Cycle 18: November 27, 1977 to December 20, 1977. Runs 654 to 711. $300 \mu \mathrm{A}$ average, $7.5 \%$ duty factor. $\mathrm{H}_{2} \mathrm{O} \cdot \mathbf{7 0 . 8}$ coulombs bio out, 44.4 coulombs b1o in.

Run Cycle 19: February 4, 1978 to March 27, 1978. Runs 766 to 800. $300 \mathrm{HA}$ average, $6 \%$ duty factor. $\mathrm{H}_{2} \mathrm{O}$ 220.7 coulombs bio out, 55.1 coulombs bio in. Biomed target broke February 22, during run 778. A target 1\% of the thickness of the normal target was installed March 14, and used unt1l the end of the cycle (runs 788 to 800 ). These data were combined with the target out data, as they did not show enhanced background levels at low energles. There were 62.1 coulombs of beam while this rarget was in. Run Cycle 20: June 15, 1978 to july 23, 1978. Runs 821 to 857. $360 \mu \mathrm{A}$ average, $7.5 \%$ duty factor. $\mathrm{D}_{2} \mathrm{O} \cdot 159.5$ coulombs b1o out, 102.1 coulombs bio in.

Run Cycle 21: August 4, 1978 to September 24, 1978. Runs 865 to 913. $360 \mu \mathrm{A}$ average, $6 \%$ duty factor. $\mathrm{D}_{2} \mathrm{O} .240 .0$ coulombs blo out, 209.9 coulombs bio in.

Run Cycle 22: November 12, 1978 to January 2, 1979. Runs 935 to 977. $500 \mu \mathrm{A}$ average, $7.5 \%$ duty factor. $\mathrm{H}_{2} \mathrm{O}$. 322.9 coulombs b1o out, 183.7 coulombs bio 1n. Problems with targets, especially A2, at this beam intensity caused much down time, and forced some running at reduced intensity (as low as $270 \mu \mathrm{A}$ average). The 
data taken at intensities less than $400 \mathrm{in}$ average were not included.

Run Cycle 23: March 10, 1979 to May 7, 1979. Runs 992 to 1053. $450 \mu \mathrm{A}$ average, $7.5 \%$ duty factor. $\mathrm{H}_{2}$ ก. 447.1 coulombs bio out, 224.5 coulombs bio 1n. Problens with heat dissipation in blomed target forced two weeks of running at $360 \mu \mathrm{A}$ everage (March 28 to April 12, runs 1009 to 1022). These data were analyzed separately.

B. HEAVY-WATER RESULTS

The heavy-water running occurred during Cycles 20 and 21 . The results will be described in terms of $R_{D}=$ observed rate/calculated rate. Results are shown as a function of run cycle, bin size, and analysis region before known systematic backgrounds have been subtracted. These subtractions were small compared to the calculated neutrino signal for both light. and heavy water, making this a valid procedure. Table XII shows the results cycle by cycle, using 5-MeV binning and analyzing from 25 to $55 \mathrm{MeV}$. The errors are purely

TABLE XII

$\mathrm{D}_{2} \mathrm{O}$ RESULTS, CYCLE BY CYCLE

Run Cycle
$\underline{R_{D}}$, B10 Out

$1.75 \pm 0.59$

$0.99 \pm 0.43$ 
statistical. No subtraction of beam-associated backgrounds has been made. The two cycles are consistent with each other, with a $x^{2}$ of 1.1 for one degree of freedom.

Table XIII shows the value of $R_{D}$ for the total $D_{2} 0$ running, for: different bin sizes and analysis regions. It can be seen that the data are not sensitive to these variations. Other systematic effects are discussed below.

Figure 40 is the total subtracted $D_{2} 0$ spectrum for biomed target out, with the expected spectrum plotted on it.

TABLE XIII

$\mathrm{D}_{2} \mathrm{O}$ RESULTS AS A FUNCTION OF BIN SIZE

AND ANALYSIS REGION

\begin{tabular}{|c|c|c|}
\hline Bin Size & Limits & $\mathrm{R}_{\mathrm{D}}$, Bio Out \\
\hline 5 & $25-55$ & $1.25 \pm 0.34$ \\
\hline 5 & $20-55$ & $1.31 \pm 0.33$ \\
\hline 5 & $30-55$ & $1.34 \pm 0.38$ \\
\hline 5 & $25-60$ & $1.25 \pm 0.34$ \\
\hline 4 & $26-54$ & $1.20 \pm 0.35$ \\
\hline 4 & $30-54$ & $1.22 \pm 0.34$ \\
\hline 8 & $24-56$ & $1.11 \pm 0.34$ \\
\hline
\end{tabular}




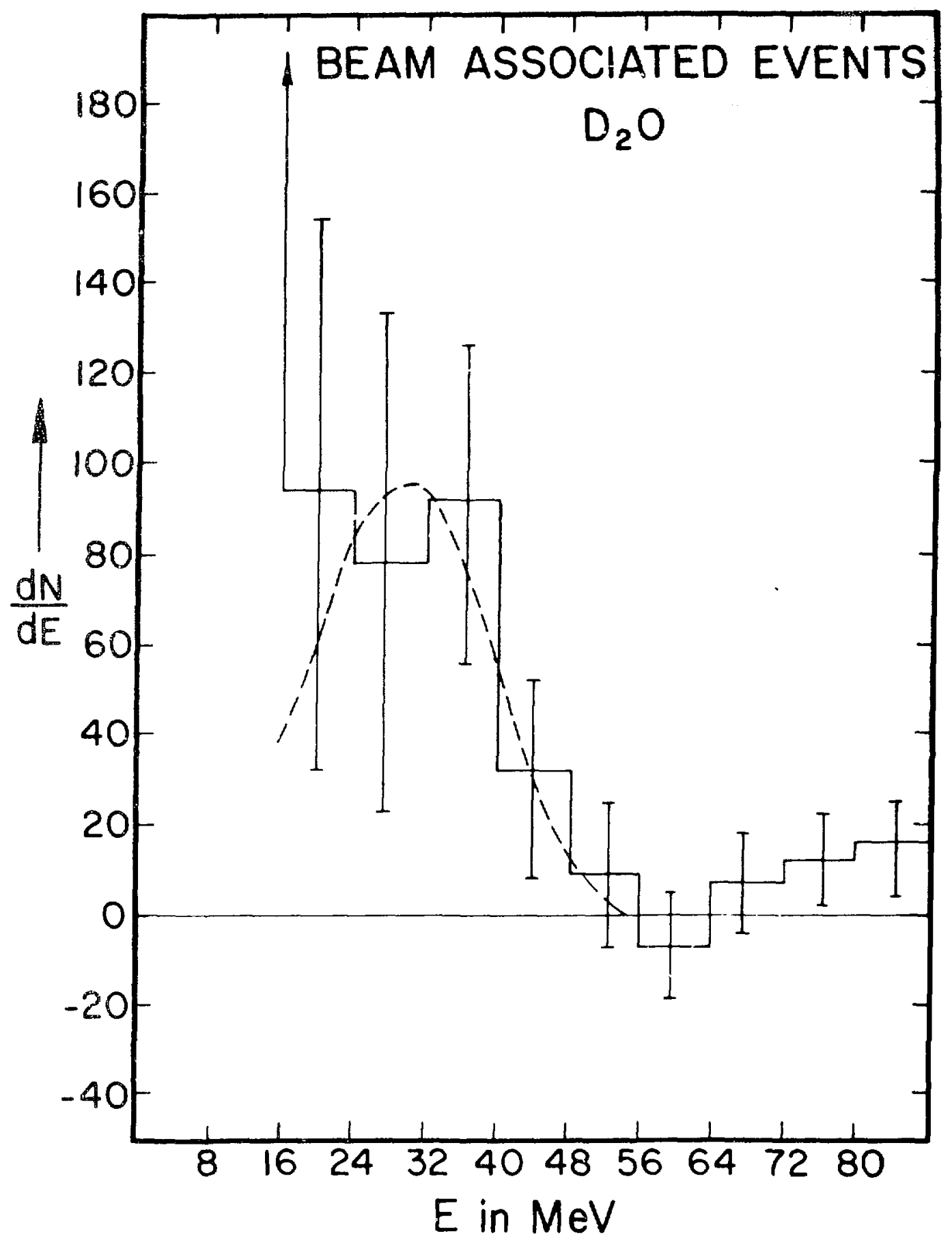

Fig. 40. $D_{2} O$ subtracted spectrum with calculated spectrum. 


\section{WATER RESULTS}

The water runing occurred during Cycles $16-19$ and 22-23. The results wil be described in terms of $R_{H}$, defined as observed rate/calculatea rate (for $R=1$ ). Table XIV shows the data cycle by cycle, using 5-MeV bianing and analyzing from 25 to $55 \mathrm{MeV}$. As In the $D_{2} \mathrm{O}$ data, the errors are purely otatistical and no subtraction of beam-assoclated backgrounds has been made. The data from the separate run cycles are consistent with each other, with a $x^{2}$ of 11.2 for $81 x$ degrees of freedom.

\section{TABLE XIV}

$$
\mathrm{H}_{2} \mathrm{O} \text { RESULTS CYCLE BY CYCLE }
$$

Run Cycle

16

17

18

19

19

22

23
$\mathrm{R}_{\mathrm{H}}$, Bio Out

$0.61 \pm 0.22$

$0.27 \pm 0.20$

$-0.26 \pm 0.20$

$-0.01 \pm 0.11$

$1 \%$ Biomed target

$-0.17 \pm 0.20$

$0.04 \pm 0.09$

$0.01 \pm 0.06$ 
Table XV shows the value of $R_{H}$ for the total $\mathrm{H}_{2} \mathrm{O}$ running for different anelysis regions and bin sizes. The data are not sensitive to these variations. Figure 41 shows the subtracted $H_{2} \mathrm{O}$ spectrum, blomed target out, and the expected spectrum for $R=1$.

\section{SYSTEMATIC EFEECTS}

As discussed in the chapter on backgrounds, there were some known contributions to a beam-associated signal which did not come from neutrino interactions on protons or deuterons. Two of these will be subtracted, the residual neutron backgrounds and the contribution from neutrino-nucleus interactions. All results, unless otherwise stated, will be for 25 to $55 \mathrm{MeV}$ and 5-MeV bins.

TABLE XV

$\mathrm{H}_{2} \mathrm{O}$ RESULTS AS A FUNCTION OF BIN SIZE AND ANALYSIS REGION

\begin{tabular}{|c|c|c|}
\hline Bin Size & Limits & $\mathrm{R}_{\mathrm{H}}$, B10 Out \\
\hline 5 & $25-55$ & $0.028 \pm 0.043$ \\
\hline 5 & $20-55$ & $0.045 \pm 0.043$ \\
\hline 5 & $30-55$ & $0.045 \pm 0.045$ \\
\hline 5 & $25-60$ & $0.028 \pm 0.043$ \\
\hline 4 & $26-54$ & $0.015 \pm 0.043$ \\
\hline 4 & $22-54$ & $0.027 \pm 0.043$ \\
\hline 3 & $24-54$ & $0.019 \pm 0.043$ \\
\hline
\end{tabular}




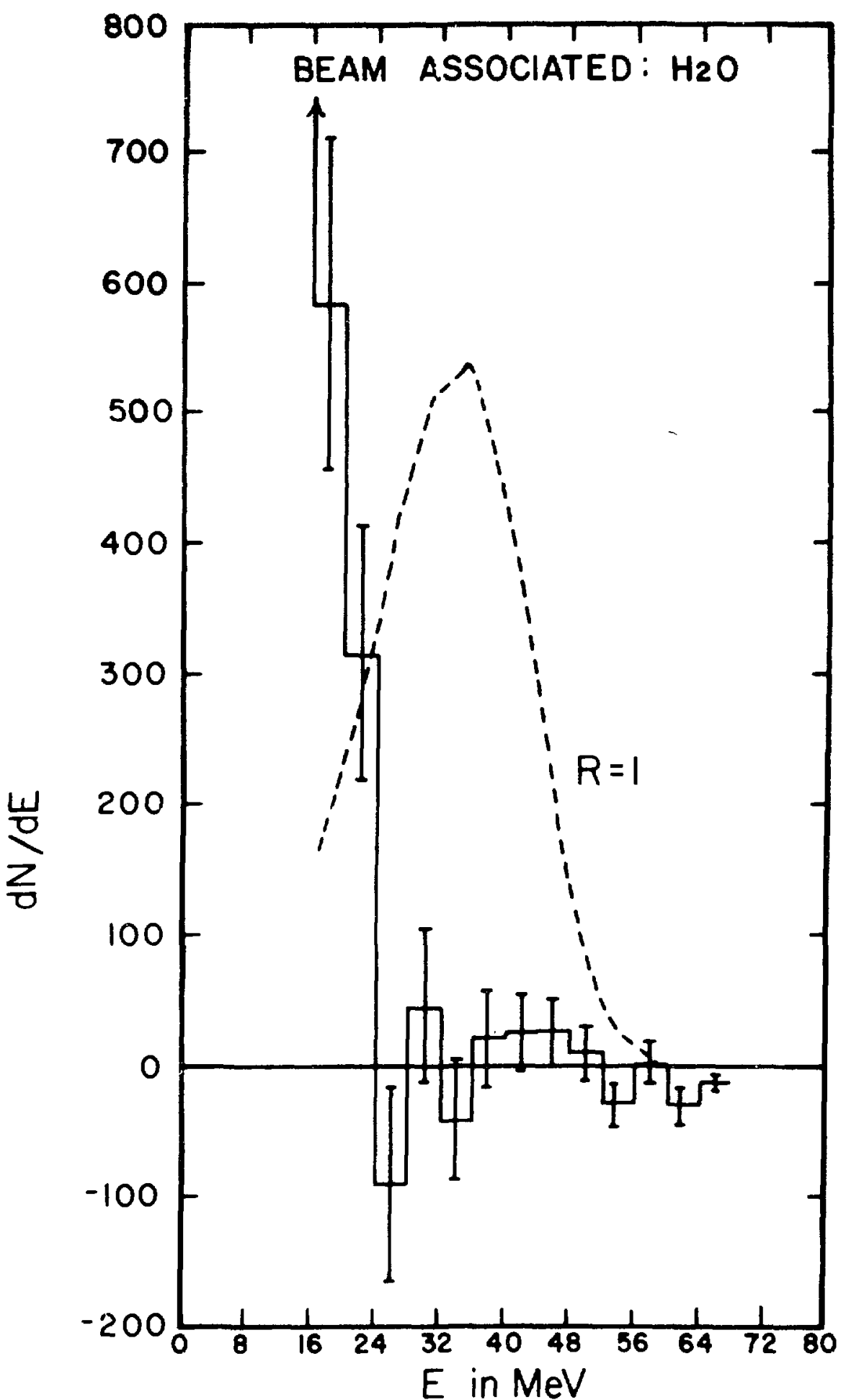

Fig. 41. $\mathrm{H}_{2} \mathrm{O}$ subtracted spectrum with calculated spectrum. 


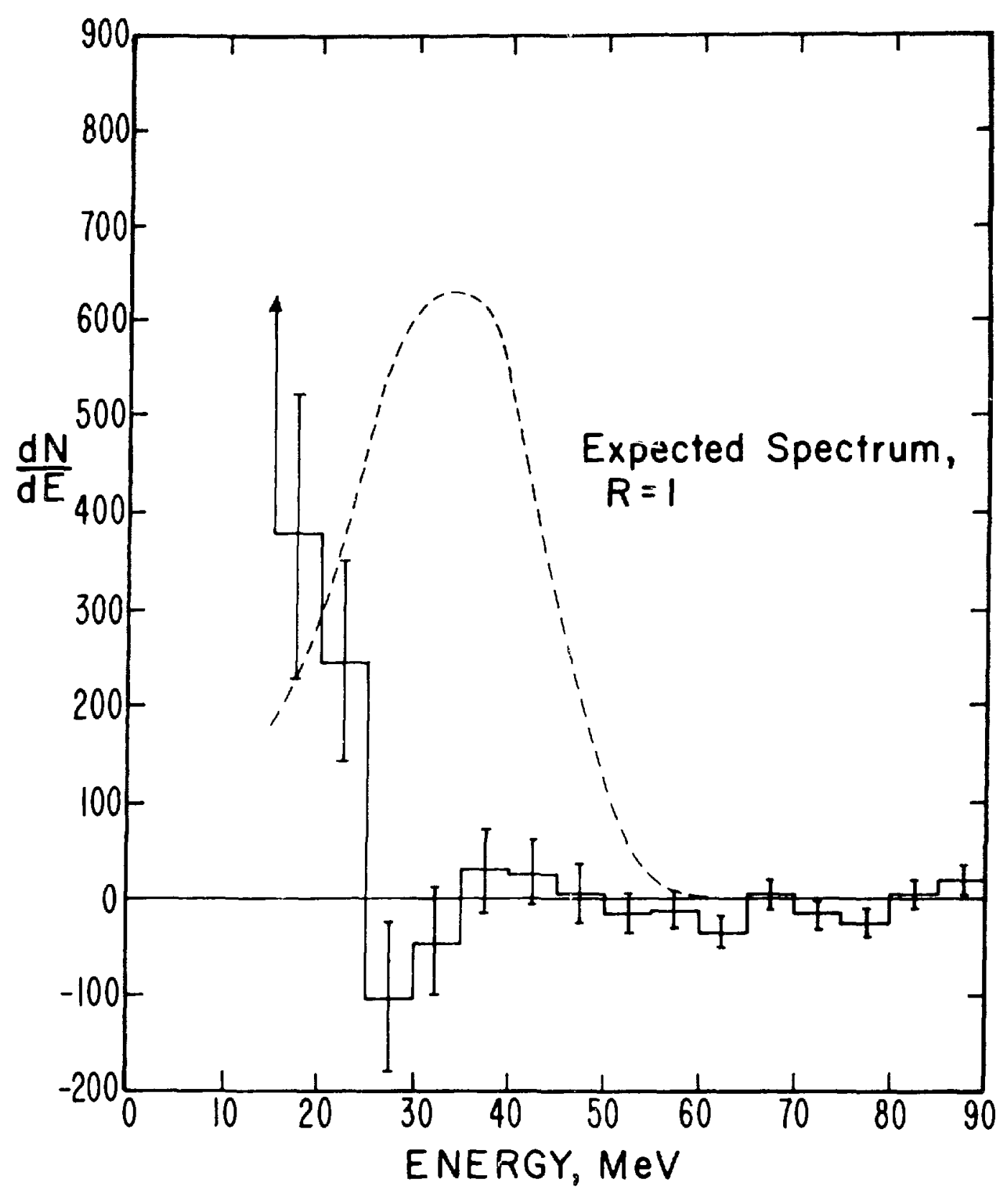

Fig. 42. $\mathrm{H}_{2} \mathrm{O}$ spect rum with neutrino-nucleus and neutron backgrounds subtracted, with calculated spectrum. 
The neutron background is shown in Figs. 28 and 29. The spectrum shape shown in Fig. 29 was normalized to the rate shown in Fig. 28, and then subtracted from both the water and the heavy water data. This gives

$$
\begin{aligned}
& R_{D}=1.11 \pm 0.34 \\
& R_{H}=0.003 \pm 0.043 .
\end{aligned}
$$

The neutrino-nucleus cross sections are given in the theoretical section, and the relative amounts of ${ }^{12} \mathrm{C},{ }^{16} \mathrm{O}$, and ${ }^{27} \mathrm{Al}$ are given in the section on backgrounds. The spectrum shapes are shown in Fig. 30 . Subtracting those spectra with the appropriate weights gives

$$
\begin{aligned}
& R_{D}=1.09 \pm 0.34 \\
& R_{H}=-0.001 \pm 0.043 .
\end{aligned}
$$

Figure 42 shows the $\mathrm{H}_{2} \mathrm{O}$ data after these subtractions have been made, with the calculated spectrum for $R=1$. Figure 43 is the same for $D_{2} 0$. The effects of other possible systematic errors on $R_{D}$ and $R_{H}$ have also been investigated. These include a normalization error on the cosmic ray subtraction, an error in the energy scale, and a shift of the zero point in the spectrum. These checks have all been made on $R_{D}$ and $R_{H}$ before the above subtractions were done. A $0.2 \%$ error in the normalization, consistent with the overall DTM subtraction given with the calibrations and checks, increased $R_{D}$ from 1.25 to 1.29 , or $3 \%$, and increased $R_{H}$ from 0.028 to 0.036 , or $29 \%$. A change in the zero position of 1 channel, which is similar to a change in gain of $3 \%$, changed $R_{D}$ 


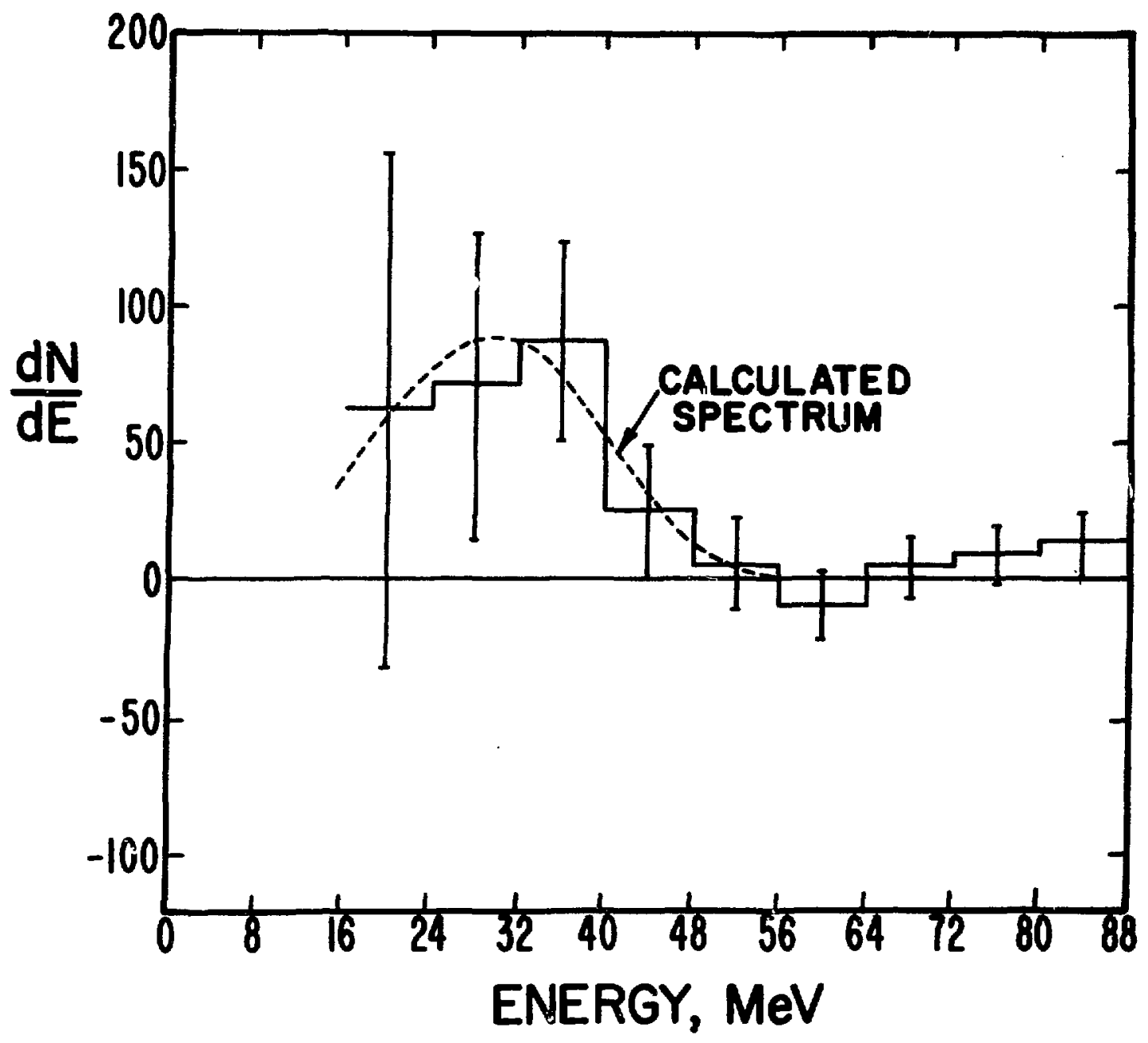

Fig. 43. $D_{2} \mathrm{O}$ spectrum with neutrino-nucleus and neutron backgrounds subtracted, with calculated spectrum. 
from 1.25 to 1.29 , or $3 \%$, and increased $R_{\mathrm{H}}$ from 0.028 to 0.036 , or $29 \%$. A change in the zero position of 1 channel, which is simllar to a change In gain of $3 \%$, changed $R_{D}$ from 1.25 to 1.31 , or $5 \%$, and changed $R_{H}$ from 0.028 to 0.027 , or $4 \%$. We therefore increase the errors on $R_{D}$ an $R_{H}$ in quadrature by these amounts; the error on $R_{D}$ increases to 0.35 and the error on $R_{H}$ t. 0.044 . We now have

$$
\begin{aligned}
& R_{D}=1.09 \pm 0.35 \\
& R_{H}=-0.001 \pm 0.044
\end{aligned}
$$

uncertalnties in the flux normalization will cancel when $R_{H}$ is renormalized by $R_{D}$. We would like to use $R_{D}$ to determine a cross section for the reaction $v_{e}{ }^{+}+p_{p} e^{-}$, however, so for that purpose its error needs to be increased to include uncertainties in the flux. Assuming a $10 \%$ uncertainty in the flux gives

$$
R_{D}=1.09 \pm 0.37
$$

The data taken when the biomedical target was in had a substantially increased neutron background; they may therefore be indicative of problems in the rest of the data. Analyzing these data in terms of $R_{D}$ and $R_{H}$, without making any of the corrections described above, yields

$$
\begin{aligned}
& R_{D}=3.07 \pm 0.50 \\
& R_{H}=0.235 \pm 0.089
\end{aligned}
$$

for the region 25 to $55 \mathrm{MeV}$ and 5-MeV bins. These numbers are consistent with the assumption that they contain the same neutron 
consistent with the assumption that they contain the same neutron contribution, even though the apparent effect on the $D_{2} \mathrm{O}$ number is wuch larger, since the neutrino cross section on deuterium is smaller than an hydrogen. Subtracting $R_{D}$, bin by bin, as measured with bio out gives a neutron contribution to blo in of $1.99 \pm 0.51$. Analyzing this neutron spectrum in terms of $R_{d}$ yields $0.42 \pm 0.10$, in good agreement vith the observed value. The following is a crude calculation of the $\bar{v}_{\mathbf{e}}$ contribution to the subtracted spectrum from $\mu^{-}$decay. We begin with the fraction of pions produced in various energy intervals by $740 \mathrm{MeV}$ protons. ${ }^{67}$ The energy scale is compressed by a factoi of 2 to take into account energy loss in the isotope production stringers upstream of the beam stop. These fractions are in the second column of Table XVI, labeled $F(T)$. The third column in this table is the fraction of pions which decay during the slowing-down time in copper, labeled $f$. The product of $F(T)$ and $f$ is shown in the fourth column, labeled $\Delta N$. Summing this column gives the fraction of pions which decay. We assume here that $\pi^{-}$which do not decay while slowing down are captured, as th. lifetime after capture is about $10^{-13}$ sec. 68 This sum is $0.0032 ; 0.32 \%$ of the $\pi^{-}$will decay. The fraction of $\mu^{-}$which will decay instead of being captured is 0.074 .69 Multiplying this by 0.0032 gives 0.00024 as the fraction of $\pi^{-}$which will eventually give a $\mu^{-}$decay. We divide this by 4 because the $\pi^{-} / \pi^{+}$ production ratio is $1 / 4,70$ giving a ratio of $\mu^{-}$decays to $\mu^{+}$decays of 0.0001 , or $1 \times 10^{-4}$.

67. D.R.F. Cochran et al., Phys. Rev. D 6, 3085 (1972).

63. R. Marshak, Meson Physics, McGraw-Hi11, 1952, p. 170.

69. M. Eckhause et al., Nuc1. Phys. 81, 575 (1966).

70. Cochran et a1., Op. Cit. 
Table XVI

$\begin{array}{cccc}\mathrm{T}_{\pi}, \mathrm{HeV} & \mathrm{F}(\mathrm{T}) & \mathrm{f} & \Delta \mathrm{N} \\ 0-10 & 0.10 & 0.0007 & 0.00007 \\ 10-50 & 0.42 & 0.0014 & 0.00059 \\ 50-100 & 0.28 & 0.0030 & 0.00084 \\ 100-200 & 0.20 & 0.0085 & 0.00170\end{array}$

This, then, is negligible compared to our level of sensitivity, and we therefore dc not correct the subtracted number for it.

\section{E. CONCLUSIONS}

Using the number for $R_{D}$ of $1.09 \pm 0.37$, we can derive a cross gection for the reaction $\nu_{e} d+\mathrm{ppe}^{-}$. This is

$$
\sigma\left(\nu_{\mathrm{e}} \mathrm{d}^{+} \mathrm{pp \textrm {e } ^ { - }}\right)=(0.52 \pm 0.18) \times 10^{-40} \mathrm{~cm}^{2}
$$

Th1s reaction is the inverse of the reaction $\mathrm{ppe}^{-}+d \nu_{e}$, which is a secondary source of energy in the sun, and is closely related to the reaction pp $\rightarrow \mathrm{de}^{+} \nu_{e}$, which is the primary source. Neither of these reactions has been seen in the laboratory. ${ }^{71}$ ours is the first measurement of the neutrino-deuteron reaction, and is in good agrement with the calculated value of $0.48 \rightarrow 10^{-40} \mathrm{~cm}^{2}$ given in the theoretical section.

71. J. S. O"Connell, op. cit. 
Using the data from 25 to $55 \mathrm{MeV}$, we have

$$
R_{D}=1.09 \pm 0.35, R_{H}=-0.001 \pm 0.044
$$

Renormalizing $R_{H}$ by $R_{D}$ as discussed above, we find

$$
R=-0.001 \pm 0.040
$$

Forming a $90 \%$ confidence level upper limit gives

$$
R<0.065(907 \text { C.L. ) . }
$$

We therefore see no evidence for a multiplicative law. The theory of Derman discussed above is not ruled out; the data may be used to put a lower limit of the mass of the $\pi=-1$ Higgs boson of

$$
\mathrm{M}_{\mathrm{H}}>3.5 \mathrm{GeV}
$$

(using $\left.\mathrm{m}_{\tau}=1782 \mathrm{MeV} / \mathrm{c}^{2}\right)^{72}$.

In order to place an upper limit on neutrino oscillations, it is necessary to integrate the expression for $w_{v_{l}, v_{l}}$, found in the theoretical section over the finite detector size and the neutrino energy spectrum. This cannot be done analytically, so it was done with a Monte Carlo. The results of the Monte Carlo are shown in Fig. 44. 


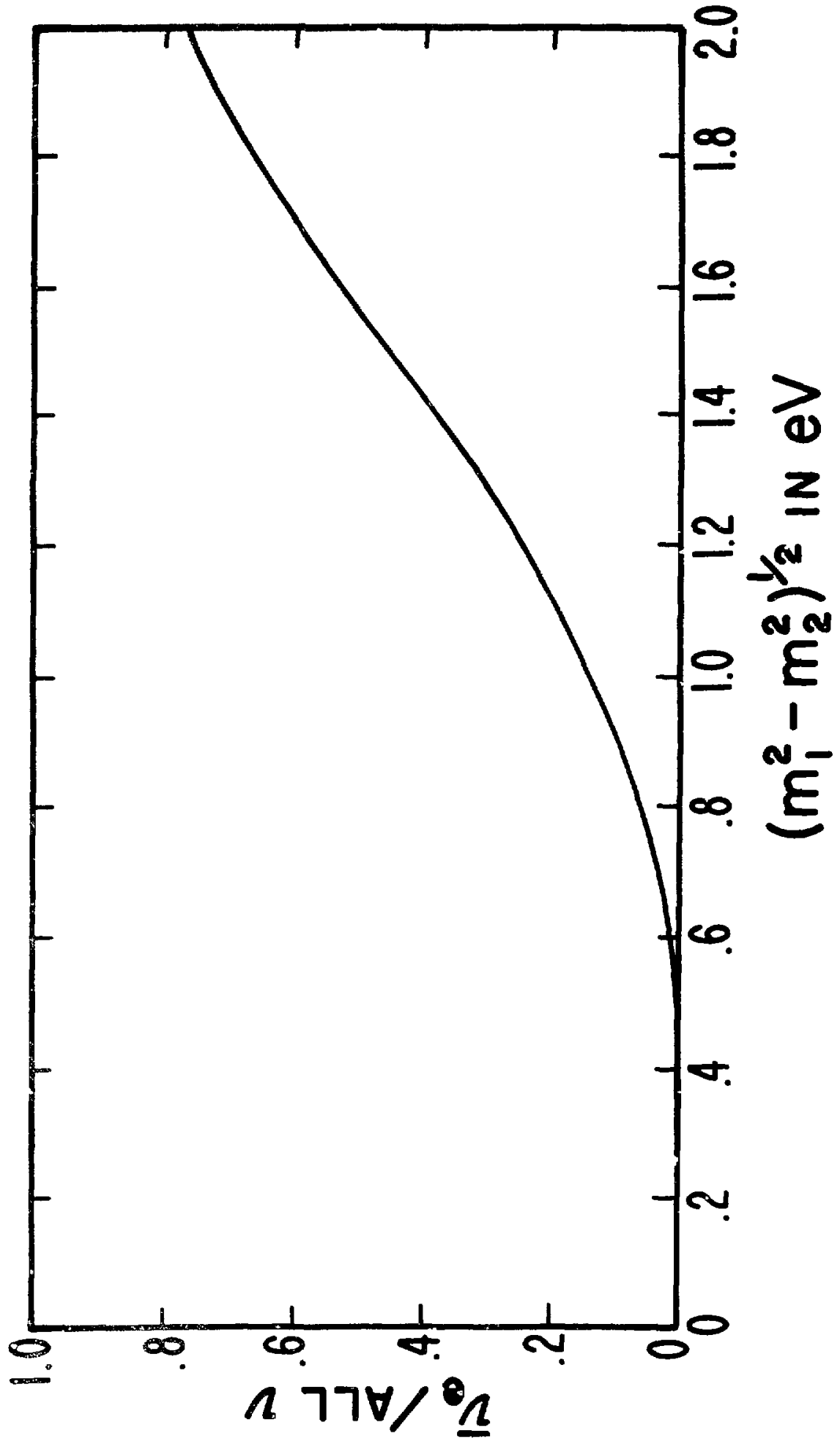

Fig. 44. Probability of observing $\bar{v}_{e}$ as a function of $M_{1}$. 
Assuming $m_{2}=0$, the present result of $R \leq 0.065$ corresponds to a limit on the netitrino mass of

$$
m_{1} \leq 0.8 \mathrm{ev}
$$

Present upper limits on this come from the Gargamelle collaboration; they report a limit of $m_{1} \leqslant 1 e v .73$

73. E. Bellotti et al., Nuov. Cim. Lett. 17, 553 (1976). 
ACKNOWLEDGEMENTS

An experiment of this length and complexity necessarily involved the contributions of many people.

I would like to thank my collaborators; they were Drs. R. L. Burman, D. R. F. Cochran, J. Duclos, J. Frank, C. K. Hargrove, V. W. Hughes, H. Kaspar, U. Moser, P. Nemethy, and R. P. Redwine. I am particular1y indebted to Peter Nemetiny, the spokesman for the experiment, whose help, guldance, and patience were essential.

Special thanks go to the LAMPF accelerator operators, and their Chief, Dr. D. C. Hagerman, who provided us with two years of beamtime. Also, the support of the LAMPF administration, particularly Dr. Louis Rosen, was invaluable.

The staffs of both LAMPF and Yale provided valuable technical support for this experiment. I am particularly grateful to the people at the LAMPF Area-A Manager's office for their help. My thesis advisor for this experiment was Dr. Vernon W. Hughes. 